\title{
Predicting Experimental Results for Polyethylene by Computer Simulation
}

\author{
J. Ramos, J.F. Vega and J. Martínez-Salazar* \\ BIOPHYM, Department of Macromolecular Physics, Instituto de Estructura de la \\ Materia, IEM-CSIC, C/ Serrano 113 bis, 28006 Madrid, Spain
}

*corresponding author: j.msalazar@csic.es 


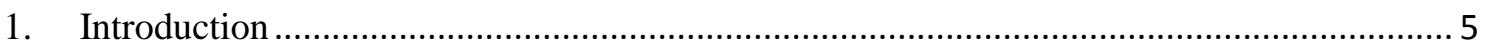

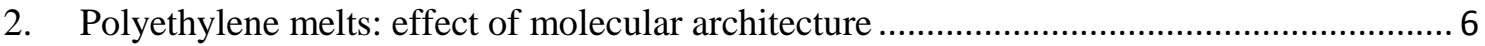

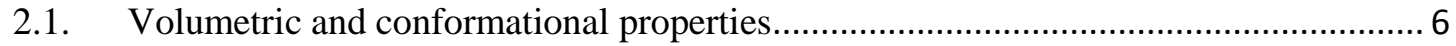

2.1.1. Volumetric and thermal expansion coefficients ................................................. 6

2.1.2. Coil dimensions and temperature coefficients ..................................................... 9

2.1.3. Local packing and static structure factor.......................................................... 14

2.1.4. Topological analysis: primitive paths and entanglements .................................. 15

2.2. Dynamic properties: entanglement relaxation time and diffusion .............................. 19

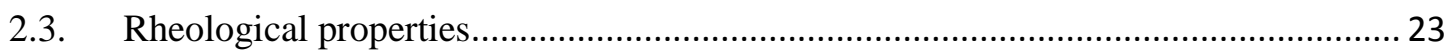

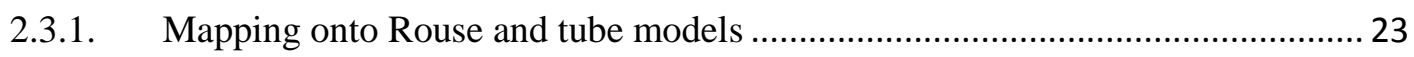

2.3.2. Non-equilibrium molecular dynamics ........................................................... 26

3. Thermal transitions: glass transition and crystallization .................................................... 27

3.1. Glass transition temperature of semi-crystalline polyethylene ….............................. 27

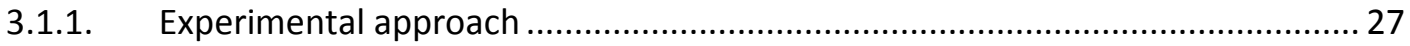

3.1.2. Computer simulation approach ...................................................................... 30

3.2. The crystalline structure of polyethylene ………….................................................. 31

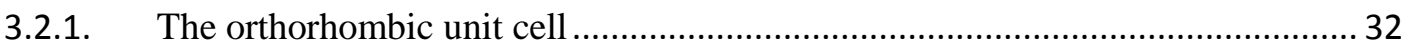

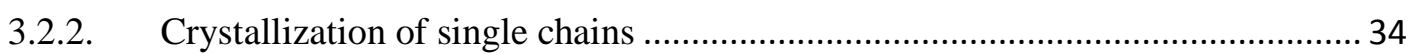

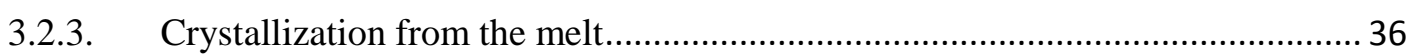

3.2.4. Nature of the amorphous region in lamellar polyethylene ................................ 43

4. Brief description of the computational methodologies used ........................................... 45

4.1. Quantum scale. Ab initio quantum (QM) and density functional theory (DFT)

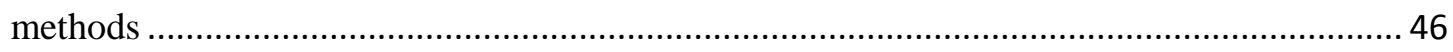

4.2. Atomistic models based on classic mechanics ........................................................ 47

4.2.1. Molecular mechanics (MM): optimization algorithms ........................................ 48

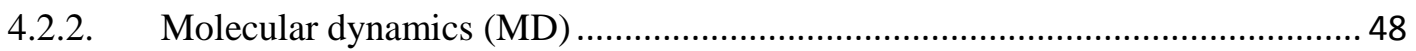

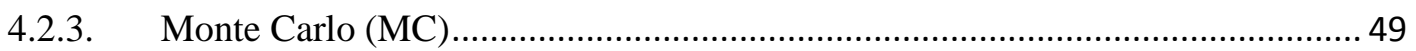

4.2.4. The problem of equilibrating long polyethylene chains..................................... 51

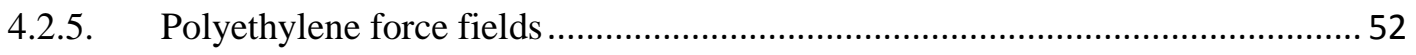

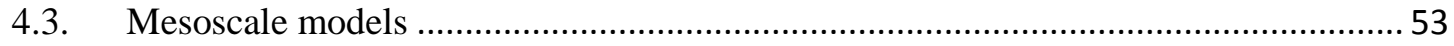

4.3.1. Coarse-grained molecular dynamics (CGMD) ................................................. 54

4.3.2. Ultra coarse-grained stochastic dynamics (CGSD) ........................................... 55

4.3.3. Dissipative particle dynamics (DPD) .............................................................. 56

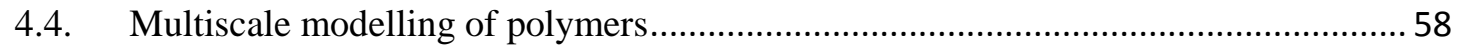

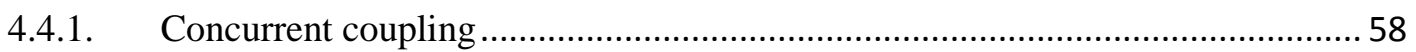


4.4.2. Hierarchical or sequential coupling.

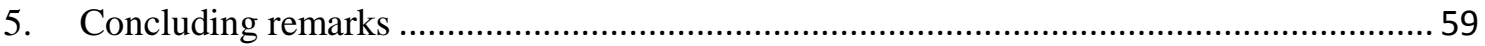




\begin{abstract}
This feature article reviews several aspects of computational approaches to polyethylene melt and solid state properties in relation to existing experimental results. Based on 40 years of experience in the field, we offer a personal view of how computer simulations are helping to understand the physics of polyethylene as a model polymer. The first issue discussed is the molten state of polyethylene, including static and dynamic properties and entanglement features along with their impacts on rheological behaviour. We then examine the glass transition, crystallization process and solid state structure, including the interlamellar region. This is followed by brief descriptions of the latest advances in simulating mechanical properties and of the various methodologies used to simulate the physics of polyethylene. Throughout the manuscript, references are made to our own work and also to studies by many other authors that have nicely contributed to developments in simulating the physics of polyethylene in close agreement with experimental results.
\end{abstract}

Keywords: Polyethylene; molecular architecture; branching; dynamics; crystallization; computer simulations 


\section{Introduction}

Polyethylene [abbreviated PE; IUPAC name polyethylene or poly(methylene)] is the most common plastic, its global annual production being around 82 millions of tonnes [1]. Many kinds of PE are known and these generally consist of a mixture of similar polymers of ethylene.

With regards to polymer physics, PE is undoubtedly the most studied polymer. The reasons for this are its chemical simplicity, its high level of production in the last 50 years and the availability of model materials obtained by different synthetic routes (long chain paraffins [2], single-site catalyst PE that include modelling reactions [2-21] and hydrogenated polybutadienes [22-24]. In addition, architecture is generally well characterized. This has meant that to a greater or lesser extent, the study of PE in the melt or solution, its solid state structure, morphology or physical properties have been the target of the polymer community for over five decades.

There are many types of PE depending on the polymerization procedure used to generate the polymer, and there are also different classifications based on their density. However, from a strictly physical point of view, several main factors control the physical properties of PE and these are the focus of interest of this article. In this regard, we recognize that the role of branches is a key factor. In this article, we consider $\mathrm{PE}$ as the large family of polymers that have the monomer ethylene - $\left(\mathrm{CH}_{2}-\mathrm{CH}_{2}\right)-$ as their main constituent, and several side branches which can be classified as long or short. Long chain branching (LCB) in PE is used to describe the presence of side branches whose molecular weight $\left(\mathrm{M}_{\mathrm{w}}\right)$ is above the critical molecular weight between entanglements $\left(\mathrm{M}_{\mathrm{e}}\right)$ but that are not as long as the main chain [25-28]. By short chain branching (SCB), we understand the presence of side branches of up to 6 carbon atoms (1-octene comonomer), and this is a feature of commercially available PE. In between, we find architectures with branches of $\mathrm{M}_{\mathrm{w}}$ below $\mathrm{M}_{\mathrm{e}}$. We also consider that branches are randomly placed along the main backbone. In this context, it should be noted that SCB is important for crystallization and solid state properties, while LCB is a determinant of rheological properties with impacts on processing.

As a third point, we should emphasize that several theories of polymers have been applied to PE. Crystal growth theories, crystallographic distortions and mechanical properties are generally applicable but many basic questions still remain unanswered. It should also be considered that the framework of theory describing the molten state and its dynamics is better understood than the solid state properties of PE when compared with model experimental data. Thus, while the selection of experimental results should be filtered according to the complexity of the molecular architecture of the systems, in this paper we have only focussed on experimental results for PE whose molecular architecture has been well characterized.

The points discussed above become important when we try to interpret experimental observations in PE using computer simulation techniques. Here, we review some aspects of computational approaches to PE melt and solid state properties in connection with the available experimental results. Several excellent books and reviews exist on polymer simulations and modelling where the reader can find very valuable information [29-38]. The aim of this feature article is to offer our vision of how computer simulations can help in understanding the physics of PE as a model polymer based on our 40 years of experience in the field. The article is organized into four main sections: first we examine the molten state including static and dynamic properties, entanglement features and their effects on rheological properties; next, we discuss the glass transition state, crystallization process and solid state structure, including the interlamellar phase; this is followed by a brief description of the latest 
advances in the simulation of mechanical properties; and finally we present another brief description, this time of the various methodologies used to simulate the physics of PE.

\section{Polyethylene melts: effect of molecular architecture}

The static and dynamic properties of the melt of unentangled or entangled linear, long- or short-branched (LCB and SCB, respectively), or cyclic PE are currently under intense investigation by computer simulations, both using Monte Carlo (MC) and/or atomistic and coarse-graining (CG) molecular dynamic (MD) models (see section 4.2 for a description of these techniques).

A key component of MC and MD simulations is the description of interactions among atoms and of movements relative to each other. These interactions are described by the so-called "force field" (FF). A broad variety of FFs can be found in the literature applied to n-alkanes and polyolefins (see Section 4.2.5).

In general, all-atom or explicit hydrogen FFs have been used to simulate short alkane properties and, in some cases, to validate united atoms (UA) models [39-42]. However, the presence of explicit hydrogen atoms increases the computational cost of the calculations for long chains, to such an extent that the relevant time- and lengthscales in the melt state are out of the reach of current computer capabilities. Notwithstanding, the most widely used for long chains are united atom FFs: (i) OPLSUA [43]; (ii) SKS-UA [44, 45]; (iii) PYS-UA [40, 46]; (iv) HMT-UA [47, 48]; (v) NERD-UA [49]; (vi) TraPPE-UA [50, 51] (vii) GROMOS-UA [52] and (viii) KMT-UA [53]. The description and abbreviations of these force fields are given in section 4.2.5.

\subsection{Volumetric and conformational properties}

\subsubsection{Volumetric and thermal expansion coefficients}

Density, $\rho$, and its change as a function of temperature is a fundamental property of polymers. The value of $\rho$ is very sensitive to the free volume in the polymer medium. Therefore, the volume-temperature relationship at the pressure of interest must be realistically represented in the simulations. Commonly, $\rho$, or its inverse, the specific volume, $v$, is measured as a function of pressure and temperature using the technique of dilatometry, and it is often referred to as pressure-volume-temperature (PVT) behaviour. There are few PVT results in the literature, mostly for n-alkanes, hydrogenated polybutadienes (HPB), Ziegler-Natta and single-site linear high (HDPE) and linear low density polyethylene (LLDPE) [54-56].

In Figure 1A, we provide the values of $\rho$ obtained for different chain lengths at a temperature $\mathrm{T} \sim 450 \mathrm{~K}$ from different sources, both experiments and simulations [5457]. MC and MD simulations using both TraPPE-UA and KMT-UA FFs offer an excellent description of both the densities and thermal expansion coefficients of $n$ alkanes and PE for a broad range of chain lengths and temperatures. HMT-UA also describes the density-chain length dependence, but slightly overestimates absolute values at a given chain length [58]. In the case of the results obtained at $\mathrm{T} \sim 509 \mathrm{~K}$ (data not shown), the scattering of simulated data is more pronounced, especially for results obtained using the PYS-UA FF. In effect, this FF tends to overestimate density [59, 60] in comparison with experiments and computer simulations performed with OPLS-UA [61], KMT-UA [53, 62-65] or TraPPE-UA [66-74] FFs.

Less attention has been paid to the effect of molecular architecture on volumetric properties obtained through computer simulations. Karayiannis et al. reported MC simulation of $\mathrm{H}$-shaped molecular architectures in PE using their KMT-UA model [75, 
76]. Tsolou et al. did the same for ring PE systems [65]. Interestingly, these authors found that ring PE melts reach the asymptotic regime (where no variation of $\rho$ with molecular length is observed) at lower $\mathrm{N}$ values than the corresponding linear PE melts (dashed red line in Fig 1.A). This observation was attributed to the absence of chain ends in rings, the density of the rings being always higher than that of the corresponding linear systems of similar molecular weight. These cyclic structures were also examined by Hur et al. through MD simulation using PYS-UA and SKS-UA non-bonded parameters $\mathrm{FF}$ at $\mathrm{T}=509 \mathrm{~K}$ [59]. The results obtained for $\rho$ as a function of molecular length are in qualitative agreement with those obtained by Tsolou et al. [65].
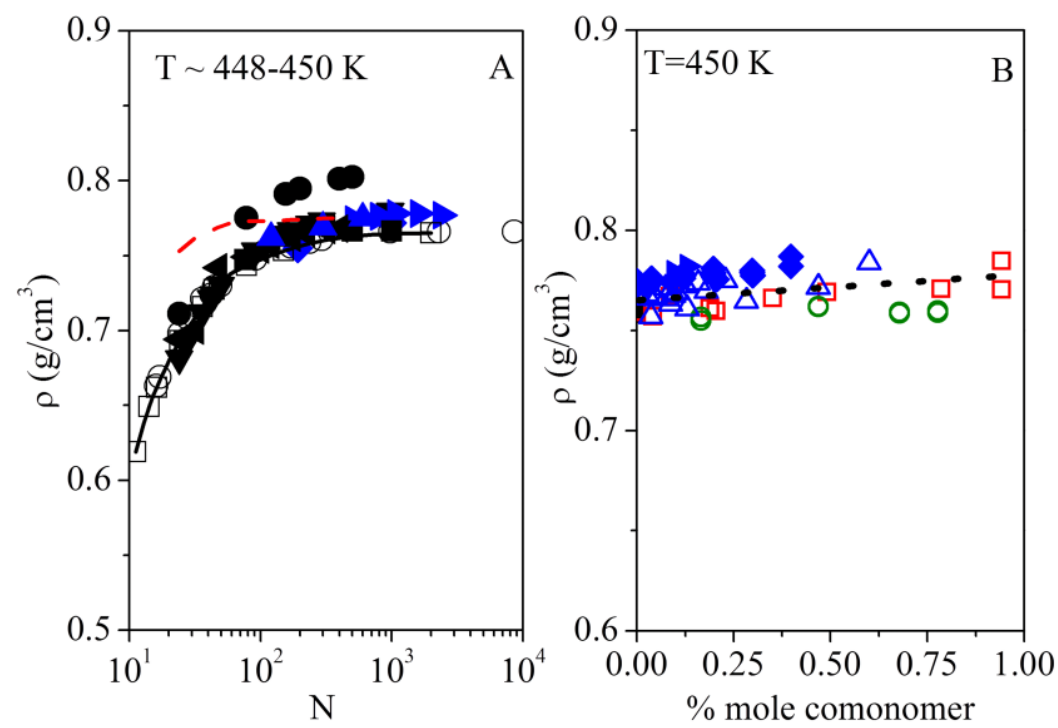

Figure 1: (A) Comparing the effects of density on chain length dependence for PE at $\mathrm{T}=448-450 \mathrm{~K}$ in experiments (open symbols and solid line) and in computer simulations (solid symbols): ( $\square$ ) Dee at al. [55]; (O) Pearson et al. [57] (data were obtained from the fit of melt rheological properties to the Rouse and reptation models using RIS calculations for the chain dimensions); (O) MC (HMT-UA) Mavrantzas et al. 1999 [58]; () MC (KMT-UA) Karayiannis et al. 2002 [53, 62]; ( $\mathbf{\Delta}$ ) MC (TraPPE-UA) Peristeras et al. 2005 [66]; ( $\nabla$ ) MC (KMT-UA) Foteinopoulou et al. [63, 64]; ( ) MD (TraPPE-UA) Ramos et al. [69-74]; ( MD (KMT-UA) Tsolou et al. [65]; ( ) MC (TraPPE-UA) Baig et al. [67, 68]. Dashed red line represents the results of simulations of ring PEs by Tsolou et al. [65]. (B) Calculated densities at 450 $\mathrm{K}$ and atmospheric pressure for linear PE and copolymers: $(\diamond) \mathrm{MC}$ (TraPPE-UA) by Ramos et al. and Moorthi et al. [69, 71] and experimental densities for ( $\square$ ) linear PE, (O) EP copolymers; ( $\square$ ) EB copolymers and $(\triangle)$ longer $\mathrm{ZN}$ and single-site copolymers by Han et al. [56] at $\mathrm{T}=450 \mathrm{~K}$ as a function of comonomer mole fraction. The dashed black line is an eye-guide line for the experimental values.

Peristeras et al. assessed the volumetric properties of tri-arm star PEs by MC simulations and TraPPE-UA FF [66]. These authors reported good agreement between their MC simulations and the experimental $\rho$ values for low density polyethylene (LDPE). Baig et al. also examined H-Shaped, pom-pom and SCB PE melts by MC simulation using TraPPE-UA FF $[67,68]$. In a systematic way, Ramos et al. explored the effects of different amounts and types of SCB (from 1-butene to 1-decene) by MC and MD also using TraPPE-UA FF $[69,71]$. In all these studies, the simulated values of $\rho$ slightly increased with the comonomer mole fraction, in agreement with the experimental results reported for different polyolefins [54-56], as can be observed in Figure 1B.

The thermal expansion coefficient at constant pressure, $\alpha$, has been also analysed by some authors. However, results so far are much more limited. From the specific volume data $(\bar{V})$ at different temperatures, $\alpha$ is defined as: 


$$
\alpha=-\frac{1}{\bar{V}}\left(\frac{d \bar{V}}{d P}\right)_{P}=\left(\frac{d \ln \bar{V}}{d T}\right)_{P}
$$

In general, simulations using KMT-UA, OPLS-UA and TraPPE-UA FFs show particularly good agreement with experiments, including a decrease in $\alpha$ with molecular

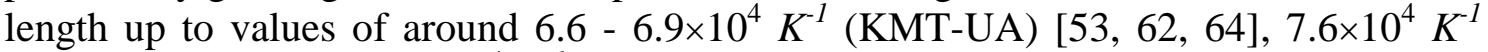
(OPLS-UA) [61] and $8.6 \times 10^{4} K^{-1}$ (TraPPE-UA) [73]. For linear high molecular weight $\mathrm{PE}$, experimental values vary between $7.6 \times 10^{4} K^{-1}$ and $8.8 \times 10^{4} K^{-1}$ [54-56]. PYS-UA underestimates the temperature dependence of density, giving rise to values around 4.75 $-5.95 \times 10^{4} K^{-1}[73]$.

No calculations of $\rho$ have been conducted at different temperatures in the case of SCB PE samples. Only in the work of Gestoso and Karayannis were values of $\rho$ at two different temperatures obtained for some SCB samples, while values of $\alpha$ were not reported [77]. Experimental $\alpha$ results for polyolefins are practically the same across the whole range of comonomer weight percent in Ziegler-Natta and single-site polyolefins, which suggests that SCB does not significantly affect the value of $\alpha$ [54-56].

Another static property of interest is the isothermal compressibility coefficient, $\beta$, defined as:

$$
\beta=-\frac{1}{\bar{V}}\left(\frac{d \bar{V}}{d P}\right)_{T}=\left(\frac{d \ln \bar{V}}{d P}\right)_{T}
$$

To estimate $\beta$, we need to obtain $\rho$ data at various pressures. Alternatively Foteinopoulou et al. [64] and Tsolou et al. [65] reported values for linear and cyclic PEs obtained from volume fluctuations of a given NPT simulation as [78]:

$$
\beta=\frac{1}{\kappa_{B} T} \frac{\left\langle V^{2}\right\rangle-\langle V\rangle^{2}}{\langle V\rangle}
$$

From both $\alpha$ and $\beta$, the solubility parameter, $\delta$, becomes available as:

$$
\delta=\sqrt{T\left(\frac{\alpha}{\beta}\right)-P}
$$

The MC simulations of Foteinopoulou et al. [64] using KMT-UA FF returned for $\mathrm{C}_{1000}$ values of $\beta=1.92 \times 10^{-3} \mathrm{MPa}^{-1}$ and $\delta=12.2 \mathrm{MPa}^{1 / 2}$ at $\mathrm{T}=400 \mathrm{~K}$ and these were compared with values obtained experimentally from PVT measurements for linear PE, $\beta$ $=0.8-0.9 \times 10^{-3} \mathrm{MPa}^{-1}$ and $\delta=18.0 \mathrm{MPa}^{1 / 2}$ at $394 \mathrm{~K}[55,56]$. Simulated $\beta$ values for ring PE melts indicated an increase in this coefficient with temperature, as for linear PE [65]. However, when we compare ring and linear PEs, it may be seen that ring topologies are less compressible than linear ones. This may be attributed to a more compact and denser structure of ring systems due to their looped structure and the complete absence of chain ends. Another interesting result of simulations is that $\beta$ varies with the molecular weight of the simulated PE rings in parallel with the variation of $\rho$.

Solubility parameter $(\delta)$ values from PVT $[56,79]$ and relative solubility parameters $\left(\delta-\delta_{\text {ref }}\right)$ from SANS experiments have been reported for a series of PEs with different amounts and types of SCB. In all cases, it was noted that $\delta$ decreases as 
the number of branches increases. This trend is well captured by simulated $\delta$ values in MC simulations on a series of PE-SCB with different amounts and types of short chain branches (see Figure 19 in [71]). In contrast, experimentally, the effect of branch length is less clear. Thus, Han et al. [56] reported that branch length did not show much influence on $\delta$ based on PVT experiments, whereas SANS-based values indicated differences among ethyl, butyl and hexyl branches. In particular, the effect of the branch was stronger for ethyl branches $\left(\left(\delta-\delta_{\text {ref }}\right) \sim \phi^{0.18}\right)$, where $\phi$ is the backbone weight fraction, in comparison with butyl and hexyl branches $\left(\left(\delta-\delta_{\text {ref }}\right) \sim \phi^{0.1}\right)$. This different scaling behaviour was captured, at least qualitatively, by the MC simulations performed by Moorthi et al. [71].

It should be noted that accurately predicting both $\alpha$ and $\beta$ as a function of the polymer topology is important as these parameters can be useful to guide the processing of polymers, such as in extrusion, moulding, and film casting. In addition $\delta$ values are related to miscibility, and can thus be used to design blends with targeted applications.

\subsubsection{Coil dimensions and temperature coefficients}

Chain dimensions are important static properties of polymers and are given by the mean-squared end-to-end distance $\left(\left\langle R_{0}^{2}\right\rangle\right)$ and the mean-squared radius of gyration $\left(\left\langle R_{g}{ }^{2}\right\rangle\right)$ of the equilibrated systems. Both $\left\langle R_{0}{ }^{2}\right\rangle$ and $\left\langle R_{g}{ }^{2}\right\rangle$ are available from MC and MD simulations of all-atomistic (AA) [80], united atoms (UA) [40, 46-48, 53, 58, 61-64, 69, 71-74, 81-88] and coarse-graining (CG) [89-97] models at different temperatures ranging from 413 to $600 \mathrm{~K}$.

Within the broad range of molecular lengths available (up to $\mathrm{N}=6,000$, where $\mathrm{N}$ is the number of carbons in the backbone) the value of $\left\langle R_{0}{ }^{2}\right\rangle / 6\left\langle R_{g}{ }^{2}\right\rangle$ decreases as the molecular weight increases and a final trend towards the Gaussian approximation $\left(\left\langle R_{0}{ }^{2}\right\rangle / 6\left\langle R_{g}{ }^{2}\right\rangle=1\right)$ is observed while the molecular weight $\mathrm{N}$ is $>150$ [98]. Significantly, near $\mathrm{N}=15$, irrespective of the use of SKS-UA [81] and PYS-UA [98] FFs, the largest deviation from Gaussian statistics (maximum $\left\langle R_{0}{ }^{2}\right\rangle / 6\left\langle R_{g}{ }^{2}\right\rangle$ ) is observed, a phenomenon noted in earlier MC simulations [99]. This observation is consistent with an elongated and rigid molecular structure of short $n$-alkanes and a broad conformational transition from a rod-like (semi-flexible) to a polymer coiled (flexible) conformation as $\mathrm{N}$ increases. Jeong and Douglas suggested its relevance to understand basic physical phenomena such as chain folding and crystallization [98]. This transition may explain failures of the Rouse model, which only applies qualitatively and questions our understanding of melt dynamics for unentangled polymers. In fact these authors suggested correlation of transition and chain dynamics with local or cooperative dynamics. Also, they raised some concerns about the use of CG of polymer chains in MD simulations, as this methodology removes internal degrees of freedom from the chains, such that local segmental phenomena may be modified.

Figure 2 displays the $\left\langle R_{0}^{2}\right\rangle$ obtained in MC and MD (UA and CG) simulations available in the literature over a broad range of molecular lengths (up to $\mathrm{N}=6,000$ ) at $\mathrm{T}$ $=423-450 \mathrm{~K}$. Further, some results at $\mathrm{T}=500-600 \mathrm{~K}$ can be found $[59,60,63,70,73$, $85,94-96]$ but these are not included in the figure. With a few exceptions, agreement among all the simulations in the log-scale is good. The results of Carbone et al. using SKS-UA in Figure 2 are slightly higher than those of the general trend, but the simulation temperature in this case is $\mathrm{T}=423 \mathrm{~K}$ [86]. In contrast, the $\mathrm{CG}$ simulations by Padding and Briels slightly underestimate $\left\langle R_{0}{ }^{2}\right\rangle$ compared to atomistic models [89, 90], as recently pointed out by Sgouros et al. [97]. All the data collected seem to follow the Flory exponent with high precision and within the limits of large $N$ (for $\mathrm{N}>150$ ), $\left\langle R_{0}{ }^{2}\right\rangle=6\left\langle R_{g}{ }^{2}\right\rangle=K \mathrm{~N}^{2 v}$, where $v=0.5$, and $K$ is a constant that depends on chain 
chemistry and temperature. Alternatively, another constant was used originally to test the molecular weight dependence of macromolecular sizes, and is given by the expression $\mathrm{R}_{\mathrm{g}}=\alpha \mathrm{M}^{v}$, thus $K=6 \alpha^{2}$.

Another characteristic parameter of polymers equivalent to the constants $K$ and $\alpha$, and widely used to test the performance of computer simulations, is the characteristic ratio, $\mathrm{C}_{\infty}$. This ratio is defined for every chain length as $\mathrm{C}_{\mathrm{n}}=\left\langle R_{0}^{2}\right\rangle / \mathrm{Nl}_{0}{ }^{2}$, where $\mathrm{l}_{0}$ is the skeletal bond distance (1.54 $\AA$ ). For Gaussian chains of infinite length, $\mathrm{C}_{\mathrm{n}}$ tends to be a constant value, $\mathrm{C}_{\infty}$. As for constants $K$ and $\alpha$, this parameter is unique for a given chemistry and temperature.

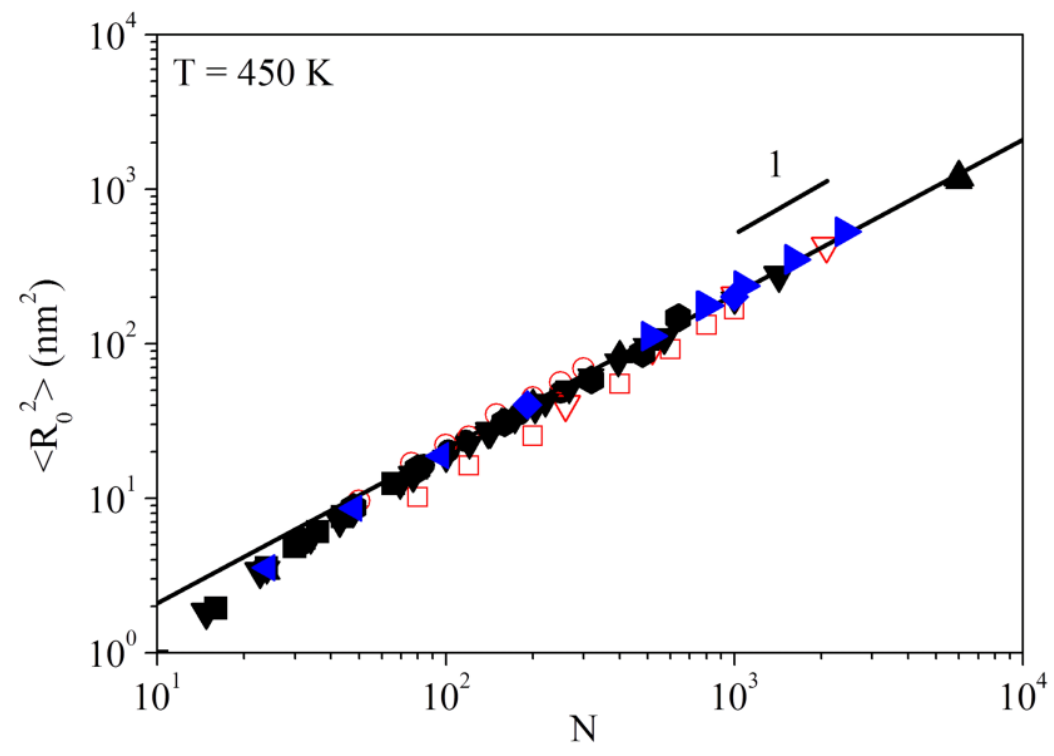

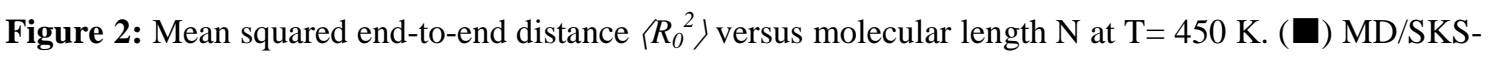
UA Mondello et al. [81]; (-) MD/HMT-UA Harmandaris et al. [47, 48, 58]; (4) MC/HMT-UA Mavrantzas et al. [58], Uhlherr et al. [82, 83]; ( $)$ MD/TraPPE-UA Heine et al. [100]; ( $\mathbf{\nabla})$ MC/KMTUA Foteinopoulou et al. [63, 64]; ( ) MC/MD/TraPPE-UA Ramos et al. [69-74]; ( ) MD/SKS-UA Carbone et al. [86]; ( $\downarrow$ MD/TraPPE-UA Baig et al. [67, 68] ; $\square)$ CG $(\lambda=20)$ Padding and Briels [89, 90]; (O) CG $(\lambda=4)$ Depa and Maranas [92] $(\mathrm{T}=423 \mathrm{~K})$; and $(\nabla) \mathrm{CG}(\mathrm{BD} / \mathrm{kMC}, \lambda=20)$ Sgouros et al. [97].

The values of $\mathrm{K}, \alpha$ and $\mathrm{C}_{\infty}$ have been repeatedly reported for $\mathrm{PE}$, mainly through viscosimetry and SANS at different temperatures (Table 1). Values of $\mathrm{C}_{\infty}=6.7-7.1$ can be obtained from indirect measurements of the radius of gyration, $R_{g}$, of linear PE fractions by means of solution viscosity measurements in $\theta$-conditions [101-103]. In this approach, the Flory-Fox equation is applied with a universal constant $\mathrm{R}_{\theta}=2.5 \times$ $10^{21}$. While the samples were carefully fractionated by precipitation, they should be considered to be relatively polydisperse [80]. This classic value is in agreement with the first SANS measurements performed in PEs by Lieser and co-workers [104]. Later, Schelten et al. reported higher values (up to $7.5 \pm 1.7$ ) also obtained by SANS [105]. Furthermore, even higher values of $\mathrm{C}_{\infty}=8.7-10.5$ were obtained by Muraoka et al. from light scattering (LS) measurements in $\theta$-conditions [106]. However, it should be noted that LS is especially sensitive to polydispersity, as the scattering intensity is proportional to the sixth power of the molecular size. Crist et al. also reported higher values of $R_{g}$ obtained by SANS than those obtained by Lieser and co-workers [107]. Later on, Horton et al. obtained a value of $\mathrm{C}_{\infty}=7.4 \pm 0.8$ in molten high molecular weight PE by SANS at $413 \mathrm{~K}$ [108]. Boothroyd et al. reported similar results in two molten PE samples in the temperature range $380 \mathrm{~K}$ to $480 \mathrm{~K}$ [109]. At $450 \mathrm{~K}$, these 
authors obtained values for $\mathrm{C}_{\infty}$ of 6.7 and 8.1 for both samples. We should, nevertheless, mention here that the SANS experiments were performed on HPB samples. The parent polybutadiene polymer is obtained by anionic polymerization and contains a minimum amount of 1.9 vinyl groups per 100 carbon atoms. Thus, the equivalent hydrogenated polymer contains 1.9 ethyl branches every 100 carbon atoms [24]. It has been established that this amount of SCB along the backbones can affect the global size of the chains $[72,74]$. The extrapolated value at $\mathrm{T}=440 \mathrm{~K}$ obtained by Fetters et al. for linear PE using results for branched HPB samples is $\mathrm{C}_{\infty}=7.2$ [23]. Zamponi et al. described $C_{n}=7.0 \pm 0.2$ at $509 \mathrm{~K}$ [110]. In summary, the variety of experimental results is fairly extensive (see Table 1 for details).

Table 1. Experimental values of $\alpha, \mathrm{K}$ and $\mathrm{C}_{\infty}$ for $\mathrm{PE}$.

\begin{tabular}{|c|c|c|c|c|}
\hline Method/Reference & Sample & $\begin{array}{c}\mathbf{\alpha}^{\mathrm{a}} \\
\AA \cdot \mathrm{mol}^{-1 / 2} \cdot \mathrm{g}^{-1 / 2}\end{array}$ & $\begin{array}{c}\mathbf{K}^{\mathrm{b}} \\
\AA^{2} \cdot \mathrm{mol}^{-1} \cdot \mathrm{g}^{-1}\end{array}$ & $\mathbf{C}_{\infty}{ }^{\mathrm{b}}$ \\
\hline VIS/[101-103] & $\begin{array}{c}\text { PE } \\
\theta \text { conditions }\end{array}$ & 0.440 & 1.16 & 6.9 \\
\hline SANS/[104] & PE (450 K) & 0.438 & 1.15 & 6.7 \\
\hline SANS/[105] & PE (423 K) & $0.46 \pm 0.05$ & $1.27 \pm 0.29$ & $7.5 \pm 1.7$ \\
\hline SANS/[107] & HPB (413 K) & $0.50 \pm 0.02$ & $1.50 \pm 0.12$ & $8.8 \pm 0.8$ \\
\hline SANS/[108] & HPB (413 K) & $0.457 \pm 0.0012$ & 1.25 & 7.4 \\
\hline SANS/[23] & HPB (440 K) & 0.449 & 1.22 & 7.2 \\
\hline SANS/[110] & HPB (509 K) & $0.443 \pm 0.005$ & 1.18 & $7.0 \pm 0.2$ \\
\hline
\end{tabular}

${ }^{\mathrm{a}}$ Measured $\mathrm{Rg}$

${ }^{\mathrm{b}}$ Assuming $\left\langle R_{0}^{2}\right\rangle=6\left\langle R_{g}^{2}\right\rangle$

The linear extrapolation at infinite molecular length of $\left\langle R_{0}^{2}\right\rangle$ has been widely used by the different authors to estimate $\mathrm{C}_{\infty}$ from computer simulations. The reader can find values obtained for $\mathrm{C}_{\infty}$ by means of $\mathrm{MC}$ and $\mathrm{MD}$ simulations at different temperatures within the ranges $\mathrm{T}=413 \mathrm{~K}-450 \mathrm{~K}$ and $\mathrm{T}=509-600 \mathrm{~K}$ ) [40, 46-48, 53, 58-64, 80, 82-92, 94-97, 100]. In general, the computed values of $\mathrm{C}_{\infty}$ (for large $\mathrm{N}$ ) at $\mathrm{T}$ $=450 \mathrm{~K}$ obtained with SKS-UA, HMT-UA and TraPPE-UA $\left(\mathrm{C}_{\infty}=9.60-8.06\right)$ are higher than those obtained using PYS-UA $\left(\mathrm{C}_{\infty}=8.5-7.1\right)$. The higher values of the characteristic ratio obtained with the former FFs have been attributed to the torsion potential employed. In effect, to solve this problem Karayannis et al. proposed a hybrid of the asymmetric UA model of Toxvaerd and the TraPPE-UA FF (KMT-UA) [53, 62]. In this case, values of $\mathrm{C}_{\infty}$ of $8.06-8.27$ can be observed for linear $\mathrm{PE}$, which is considered within the range of experimental results [88]. MD simulations of CG long $\mathrm{PE}$ models also return slightly higher values of $\mathrm{C}_{\infty}=8.93$ [97].

As for volumetric properties, little attention has been paid to the effects of both SCB and LCB on the conformational properties of PE melts. Chain contraction in branched PEs has been extensively probed experimentally. Fetters et al. assessed by SANS the melt chain dimensions of SCB PE, more precisely of ethylene/1-butene (EB) model copolymers obtained from polybutadienes by hydrogenation [23]. These authors observed that $\left\langle R_{o}{ }^{2}\right\rangle / M$ decreases monotonically as the ethyl branch content is increased. Sun et al. also reported the same trend in model ethylene/propylene (EP), EB, ethylene/1-hexene (EH) and ethylene /1-octene (EO) copolymers through SEC-LS-VIS determination of coil dimensions [111]. Neelakantan et al. investigated intermolecular packing and coil dimensions in a series of five random EB copolymers consisting of 60 united atoms by MD using the OPLS-UA FF. A decrease in $\left\langle R_{o}^{2}\right\rangle / M$ was detected with branching, parallel to that observed in experiments [112]. Ramos et al. performed 
systematic simulations using combinations of MC moves [69, 71] and MD [72, 74] using TraPPE-UA in long (1,000 main chain atoms) random ethylene/ $\alpha$-olefin copolymers with variable branching contents. In addition, Baig et al. implemented MC algorithms to simulate SCB PEs also using the TraPPE-UA FF [67, 68]. In these studies, it was shown that the distribution of trans/gauche bonds is altered by the presence of SCB. More recently, Panizon et al. presented a CG model of SCB PE for EB copolymers compatible with MARTINI that shows excellent agreement with the available data in terms of both $\rho$ and $\left\langle R_{g}{ }^{2}\right\rangle$ in the melt, and also quite well describes the contraction of the macromolecule as SCB content increases [113]. Both the experimental $[23,110,111]$ and computational $[67-69,71,72,74,113]$ results are in agreement with rotational isomeric state (RIS) model calculations, which have also shown that $R_{\mathrm{g}}$ decreases substantially as the number of branches increases [114]. Figure 3A shows a comparison of the contraction factor, $\mathrm{g}_{\mathrm{SCB}}=\left\langle R_{g}{ }^{2}\right\rangle_{B} /\left\langle R_{g}{ }^{2}\right\rangle_{L}$, obtained from experiments, computer simulations and RIS calculations on ethylene/ $\alpha$-olefin copolymers as a function of the weight fraction of branches, $S_{c}$. It can be observed that the simulations perfectly match the experiments. Only the experimental results obtained for Ziegler-Natta heterogeneous EO copolymers deviate from the general behaviour [111].
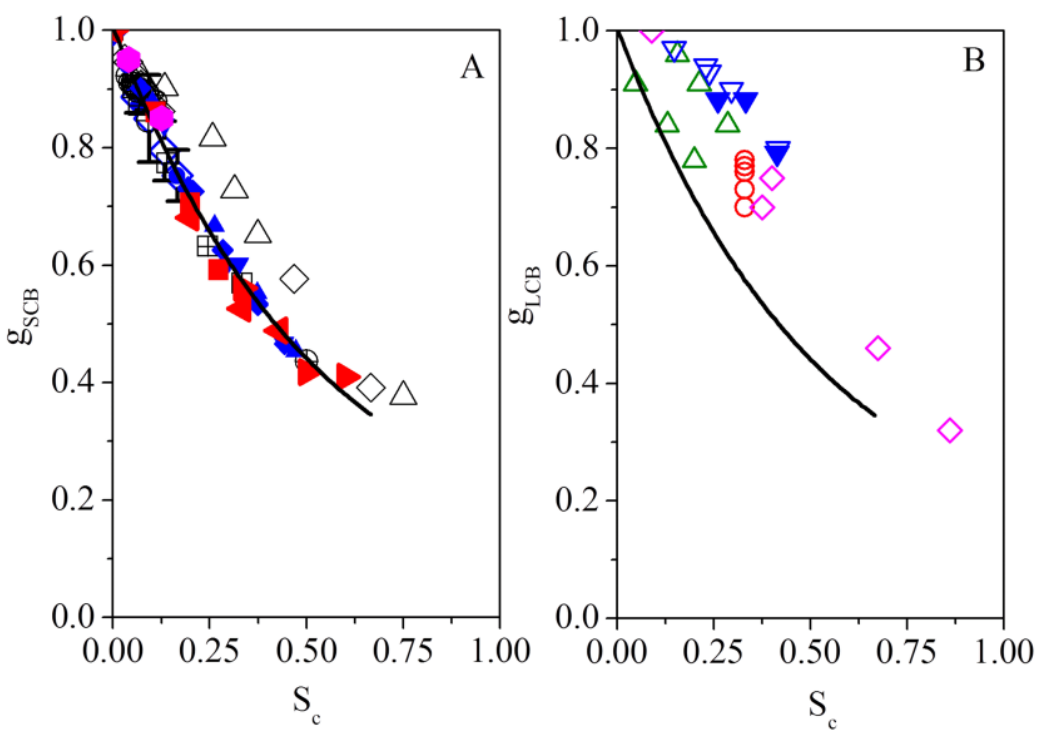

Figure 3: Experimental and simulated contraction factors of different polyolefins. (A) Effect of SCB.

Experiments by Sun et al. [111]: $(\boxplus)$ EP copolymer, $(\oplus)$ EB copolymer, $(\diamond)$ EH copolymers, $(\triangle)$ EO copolymers. Simulations by Ramos et al. [69, 71, 72, 74]: $(\boldsymbol{\bullet})$ EB copolymers, $(\bullet)$ EH copolymers, $(\boldsymbol{\Delta})$ EO copolymers, $(\boldsymbol{\nabla})$ ED copolymers. PRISM/MC calculations by Li et al. [114]: (ם) EP copolymers, (4) EB copolymers, ( ) EH copolymers. Simulations of Panizon et al. using MARTINI [113]: ( ) EB copolymers. (B) Effect of LCB. Experiments by Hadjichristidis et al. in model LCB-PE [115]: (O) symmetric stars, $(\triangle)$ asymmetric stars, $(\nabla) \mathrm{H}$, super-H and pom-pom, $(\diamond)$ Combs. Simulations by Karayannis et al. [75] and Baig et al. [67, 68]: ( $)$ H-shaped and pom-pom. The solid line represents the trend observed for SCB PEs.

For LCB, Hadjichristidis and co-workers synthesized an elegant set of linear LCB PE samples, including symmetric and asymmetric stars, $\mathrm{H}$, super $\mathrm{H}$, pom-pom and comb PE samples (HPB) [115]. These authors showed that LCB structure also controls chain dimensions, but that the Zimm-Stockmayer model only works well for describing the sizes of systems with the smaller amounts of LCB. The theory underestimates contraction factor values as obtained from GPC-MALLS and viscosimetry for the systems with the higher amounts of LCB, indicating a flaw in the available theories. 
From this view point, computer simulations can provide some insight into the conformational properties of LCB PE. Karayannis et al. reported the first MC simulation of an $\mathrm{H}$-shaped PE melt, introducing new MC moves to efficiently simulate the properties of branched macromolecules [75]. Later, Peristeras et al. used a similar approach to examine tri-arm star PE melts [66]. Baig et al. also implemented MC algorithms to simulate even more complex structures, including $\mathrm{H}$-shaped and multiarms (pom-pom) [67, 68]. Their results on LCB macromolecule contraction in comparison to their linear counterparts are in good agreement with experimental observations, as can be observed in the experimental and simulated contraction factors of different polyolefins (Figure 3B). In this case, however, there are few results from simulations regarding macromolecular conformation details in LCB PE samples. Thus, a more systematic study of the effects of molecular topology is needed to further our understanding of this important molecular characteristic of polyolefins. Of note, we should mention that SCB causes a much stronger effect than LCB on chain dimensions for a given side chain weight fraction, as illustrated in the comparison picture shown in Figure 3B.

Another interesting feature of macromolecular coil dimensions is their temperature dependence, which is given by $\kappa=\mathrm{d}\left[\ln \left\langle\mathrm{R}_{\mathrm{g}}{ }^{2}\right\rangle\right] / \mathrm{dT}$. The role of $\kappa$ on packing length and entanglement properties, such as the "plateau" modulus, $\mathrm{G}_{\mathrm{N}}{ }^{0}$, is significant. This is particularly important when melt rheology is assessed over a wide temperature range. Experimental $\kappa$ values have been obtained by means of $\theta$-condition solution measurements [102, 116, 117] and melt state experiments, including thermoelastic measurements on networks [118, 119] and SANS [23, 109]. Reported $\kappa$ values for linear $\mathrm{PE}$ vary from -1.06 to $-1.25 \times 10^{-3} \mathrm{~K}^{-1}$. Excellent agreement between experimental and simulated values is observed in the case of all atomistic simulations and the RIS model [80]. Agreement is also perfect with results obtained in MC and MD simulations performed with the TraPPE-UA FF. Foteinopoulou et al. examined this specific behaviour of $\kappa$ in linear PE chains through MC simulations and KHT-UA, obtaining an identical result [64]. OPLS-UA also seems to be a good option for this purpose [61]. The values given by the simulations performed with PYS-UA are quite different [73]. In this case lower absolute values of $\kappa$ than those expected are obtained. It has been suggested as well that an increase in the trans population of dihedral angles is the main factor determining the considerable expansion of chain dimensions of linear PE produced with decreasing temperature. However, a recent comparison between TraPPEUA and PYS-UA has shown that the difference in the trans population is small. Results indicate that not only the dihedral angle population, but perhaps also non-bonded chain interactions, could play a role in the characteristic coil sizes of the systems. In this context, it would be interesting to study the effects of both SCB and LCB on $\kappa$. A very limited set of experimental results exists in the literature. Fetters and co-workers explored the temperature dependence of unperturbed chain dimensions of $\mathrm{EB}$ copolymer by SANS [23]. In a number of cases, the values of $\kappa$ were in clear disagreement with those obtained from viscosimetry in $\theta$ conditions, but their most interesting result was a change, from negative to positive, produced in $\kappa$ as SCB contents increased, at least in EB copolymers. As discussed by Fetters and co-workers, these results indicate that the trans and gauche energy ratio changes due to the addition of side groups when going from the linear $\mathrm{PE}$, where the more compact gauche conformer has a higher energy state than the more extended trans form, to poly(1butene), where the addition of articulated side groups reverses the order leading to positive values of $\kappa$. The determination of $\kappa$ in the case of LCB PE samples could be even more interesting. 


\subsubsection{Local packing and static structure factor}

Local chain packing is generally used to characterize the organization of monomers in the melt. Melt structure can be effectively investigated by calculating the intrachain pair density function $g_{\text {intra }}(r)$ and the intermolecular pair distribution function $g_{\text {inter }}(r)$. Mavrantzas et al. assessed these functions in the case of linear PE for different chain lengths by means of MC simulations and HMT-UA FF [47, 48, 58]. Foteinopolou et al. undertook similar work using KHT-UA FF but at different temperatures [64]. Banaszak et al. [120], Neelakantan et al. [112], and Ramos et al. [69, 71] addressed the impacts of SCB using NERD-UA, OPLS-UA, and TraPPE-UA FFs, respectively. More recently, the case of linear PE was compared using both TraPPE-UA and PYS-UA FFs [73]. The effect of LCB was examined by Peristeras et al. [66] in tri-arm star PE and by Baig et al. $[67,68]$ in H-shaped and pom-pom PE.

The $g_{\text {intra }}(r)$ or $w(r)$ function reflects the characteristic distances between different pairs of polymers belonging to the same polymer chain. The maximum of the first peak at $r=1.54 \AA$ corresponds to the bond length distance (1-2 distance). The second peak, at $\mathrm{r}=2.6 \AA$, corresponds to fluctuations in the distance between atoms two bonds apart (1-3 distance). Consecutive local maxima at characteristic distances around $3.2 \AA$ and $3.9 \AA$ are attributed to different conformations of the 1-4 torsion angles, gauche and trans, with a preference towards the trans population. The positions of the peaks are independent of $\mathrm{N}$, but as the temperature decreases, the relative intensities of their maxima and depths become more prominent. At low temperatures of $\mathrm{T}=300 \mathrm{~K}$, local ordering develops as indicated by the presence of characteristic peaks at distances as long as $9 \AA$ [64]. Differences between the TraPPE-UA and PYS-UA have been detected in linear PE. Intramolecular distances at $450 \mathrm{~K}$ for the second (1-3 distance), third (1-4 gauche distance) and fourth (1-4 trans distance) peaks are more intense for the TraPPEUA as compared to those obtained using PYS-UA, and consequently, the chain dimensions calculated for the former are also higher. In the case of SCB PEs, the peak intensity in the vicinity of $3.2 \AA$ (1-4 gauche conformations) increases [71] in close agreement with the torsion distributions as a function of SCB content previously reported [69]. This denser intramolecular packing is consistent with the trend shown in Figure 1 where density increases as does SCB. This behaviour can be attributed to the coiling of backbones and a crowding effect caused by branch points.

Also, $g_{\text {inter }}$ can offer information about the structure of the molten polymers. In this case, typical broad peaks at around $r=5,10$, and $15 \AA$ are attributed to intermolecular packing and the patterns for linear PE are similar in the simulations performed with the different FFs discussed above. However, the height and position of the nearestneighbour peaks are slightly different for PYS-UA, indicating the stronger packing of chains for this model. In addition, ginter clearly shows the "correlation hole effect" and scaling with chain length $[47,48,58]$ and with temperature [64]. The positions of the peaks in $\mathrm{g}_{\text {inter }}$ at short distances shift to lower values as $\mathrm{N}$ increases and $\mathrm{T}$ decreases, being independent of molecular weight distribution. The presence of SCB strongly affects the patterns of $\mathrm{g}_{\text {inter }}$. The intensity of all intermolecular peaks decreases with SCB. Even for the maximum distance studied $(r \sim 40 \AA), g_{\text {inter }}(r)<1$, indicating large intermolecular correlation holes over distances proportional to $\mathrm{R}_{\mathrm{g}}[69,71]$. The intensity of the nearest-neighbour peak decreases as SCB increases, indicating less effective packing of the chains as the number of SCB increases. In addition, a shift to higher distances with SCB is also observed [69]. The exclusion of segments arising from other chains at distances shorter than $\mathrm{R}_{\mathrm{g}}$ becomes more pronounced as SCB increases. Thus, the most branched systems are locally denser than the less branched ones, making efficient packing of chains in the melt more difficult. In general, as branching increases, 
other chains are more strongly excluded from the surroundings of a particular chain. The effect of LCB on the pair radial distribution functions is much more subtle, but still measureable. Peristeras et al. described that $g_{\mathrm{CH} 3-\mathrm{CH} 2}(r)$ for linear PE and LCB star PE polymers is similar, whereas $g_{\mathrm{CH} 2-\mathrm{CH} 2}(r)$ is greater for the linear polymer than for the corresponding tri-arm star polymer, reflecting the fact that the LCB macromolecules are more compact than linear chains of the same molecular weight [66]. As a consequence, they show a stronger "correlation hole effect" in the melt, as in the case of SCB macromolecules. However, Baig et al. found that H-shaped and pom-pom systems are characterized by similar $g_{\text {inter }}$ curves $[67,68]$.

By combining intrachain pair density and intermolecular pair distribution functions, we obtain the total pair distribution function $g(r)$. This function is of great significance because its Fourier transform gives the static structure factor $S(q)$, which can be measured experimentally by X-ray diffraction. $S(q)$ has been seen to be insensitive to chain architecture [47, 48, 58, 64]. In general, the most frequently used FFs such as HMT-UA, KMT-UA, PYS-UA and TraPPE-UA give rise to excellent reproduction of experimental $\mathrm{S}(\mathrm{q})$ for linear PE. The comparison by Boland et al. using NERD-UA, OPLS-UA and TraPPE-UA revealed that the three FFs represent $g(r)$ equally well for linear PE [61]. Close inspection shows that the results given by KMT-UA and TraPPEUA FFs fit in exceptionally well with the experiments, as both are able to describe with high accuracy both the positions and intensities of the characteristic peaks especially of the largest first peak $[70,73]$. The small differences found in the $S(q)$ function obtained from the different FFs in terms of peak positions and intensities are in agreement with those found also in density and intermolecular distances. In contrast, it is expected that differences will emerge as a consequence of the presence of SCB, regardless of the type of branching. In fact, WAXS experiments have indicated expansion of the amorphous phase as an increase in average interchain separation with SCB occurs in both classic branched PE [121-123] and model single-site SCB PE samples [124]. This specific observation has not been yet explored systematically by computer simulations.

\subsubsection{Topological analysis: primitive paths and entanglements}

Understanding the microscopy origin of the tube and reptation model has been the focus of intensive research activity in polymer science. Within this framework, there are two basic length scales depicting the static structure of polymers: Kuhn length, $l_{K}$, and packing length, $p$. The former describes the local conformation of the chains, and is defined as the step length of a random walk with the same contour length as the polymer chains, $L$, which yields $\left\langle\mathrm{R}_{0}^{2}\right\rangle=1_{\mathrm{K}} \mathrm{L}[125,126]$. However, the random coils strongly interpenetrate each other on the $p$ scale, defined as the characteristic length scale at which coils start to interpenetrate [127]:

$$
p=\frac{V}{\left\langle R_{0}^{2}\right\rangle}=\frac{M}{\rho N_{a}\left\langle R_{0}^{2}\right\rangle}
$$

,where $\mathrm{V}$ is the volume occupied by the molecule, $\mathrm{M}$ the molecular weight, $\rho$ is the density and $\mathrm{N}_{\mathrm{a}}$ is Avogadro's number. Remarkably, $p$ is only dependent on the chemical structure of the polymer, at least for linear (without LCB) polymers, since both $\mathrm{V}$ and $\left\langle\mathrm{R}_{0}{ }^{2}\right\rangle$ (by virtue of Flory's random coil hypothesis) are proportional to $\mathrm{N}$, the polymer length. The result obtained for a wide range of polymer species links this characteristic length scale with the entanglement molar mass, $M_{e}$, having been established that $M_{e}$ is proportional to $\rho p^{3}$ [128-131]. Therefore, the packing model is directly related to the entangled melt and solution rheological behaviour by means of 
the empirical relationship, as $\mathrm{M}_{\mathrm{e}}$ and the "plateau" modulus, $\mathrm{G}_{\mathrm{N}}{ }$, are directly related [128-131]:

$$
G_{N}^{0} \sim \frac{1}{M_{e}} \sim k_{B} T p^{-3}
$$

In the case of PE, the model predicts that the decrease in $\left\langle R_{g}{ }^{2}\right\rangle / \mathrm{M}$ with the side chain weight fraction $\left(S_{c}\right)$ or, alternatively, with the average molecular weight per backbone bond $\left(m_{b}\right)$, causes an increase in $p$ and consequently a decrease in $G_{N}{ }^{0}$, an experimental fact repeatedly reported in the literature for model HPB samples [22, 79] and single-site ethylene/ $\alpha$-olefin copolymers [132-136]. This empirical fact is especially valuable in the case of polyolefins for which unperturbed dimensions remains unmeasured [131], as knowledge of $\mathrm{m}_{\mathrm{b}}$ gives a direct estimation of $G_{N}{ }^{0}$ and thus of $\mathrm{M}_{\mathrm{e}}=$ $\rho R T / G_{e}$, with $G_{N}{ }^{0}=(4 / 5) G_{e}$ in the tube theory [137]. The basic version of the reptation model applied to polymer rheology was first discussed by de Gennes [126] and developed by Doi and Edwards [125]. In this theory, restrictions in the motions of an entangled chain are modelled by confining it to a $d_{T}$ diameter tube formed by the surrounding chains. This length scale is defined in this model by:

$$
d_{T}=\sqrt{\frac{M_{e}\left\langle R_{0}^{2}\right\rangle}{M}}
$$

The problem, however, is identifying $\mathrm{d}_{\mathrm{T}}$ in a direct way, since this is not possible experimentally without considering the tube models. Edwards visualized a chain as embedded in a network of fixed obstacles representing the constraints imposed by the other polymers, and identified the random walk-like axis of the tube as what he called the 'primitive path' (PP): the shortest path between the endpoints of the chain around which its contour can be contracted without crossing any obstacle. The PP gives a direct measure of the number of entanglements, $\mathrm{N}_{\mathrm{e}}$, or in other words of $\mathrm{M}_{\mathrm{e}}$. To identify PP is of major interest, as it constitutes the missing link between the mean-field tube theories and microscopy descriptions via MC or MD simulations. To establish this connection, a number of researchers have employed computational methods to identify the PP that describes the topological state of the entangled chains. Thus, Everaers and co-workers introduced the "annealing" method for primitive path analysis (PPA) based on Edwards's definition, using a scheme for the contour reduction applied to all the chains contained in the computational sample [138]. These authors applied the method to CG FENE-type chains and reproduced $\mathrm{M}_{\mathrm{e}}$ scaling in quantitative agreement with empirical observations of the packing model given by Equation (6). Zhou and Larson proposed alternative methods of minimization to identify the PPs and their distributions [139]. Geometrical PPA in both polymer melts and entangled solutions has been performed using Z1 and CReTA codes, developed by Kröger [140] and Tzoumanekas and Theodorou [141], respectively. Both programmes construct the PPs by fixing the chain ends in the space and minimizing their contour lengths under the constraint of uncrossability. They then calculate the length of all PPs in the systems and the number of entanglements per chain, as well as the number of monomers per entanglement strand, $\mathrm{Ne}$, and consequently $\mathrm{M}_{\mathrm{e}}$. It has been well established that both topological algorithms, when applied to the same system configurations, offer almost identical results about the average contour length, $\left\langle\mathrm{L}_{\mathrm{pp}}\right\rangle$, the principal quantity of interest 
characterizing the PPs [64]. These "annealing" and geometrical approaches, also called chain-shrinking methods, reproduce a discrete view of entanglements as binary contacts, as the reduction in the chain configuration to PPs constructs straight paths between them. These methods have been applied to describe the entanglement state of UA models of linear PE of different molecular weights [64, 142-144] and also of different ethylene/ $\alpha$-olefin copolymers $[67-69,71]$. More recently, other methods that implicitly assume the tube as continuous have been developed [145-148]. In these cases, the tube is visualized by means of an iso-configurational ensemble (ICE) method of CG FENEtype chains, from which PP is obtained by averaging different chain conformations over several trajectories, and the tube confining potential is extracted from the positions of the monomers surrounding the PP.

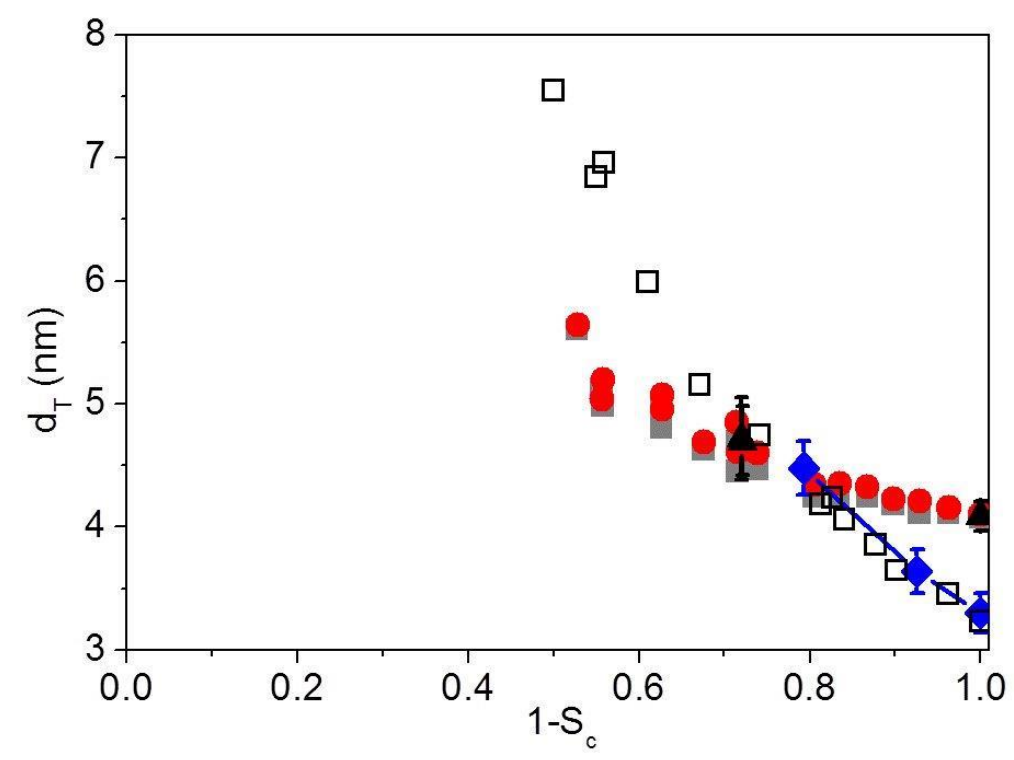

Figure 4: Effect of SCB on tube diameter, $\mathrm{d}_{\mathrm{T}}$ : $(\square)$ Experimental trend for EB copolymers (HPB) by Lohse [79]; Simulations using ( $\mathbf{\square}$ ) Z1 code and (O) CReTa code by Moorthi et al. [71]; MD/TraPPE-UA simulations by Ramos et al. [72, 74] and (A) Z1 code by Baig et al. [64, 142]

Yet another alternative method exists to determine $\mathrm{N}_{\mathrm{e}}$ or $\mathrm{d}_{\mathrm{T}}$, namely the MD/MSD methodology proposed by Kremer et al. [149-151] and later by Likhtman and McLeish [137]. This method has been used to calculate $d_{T}$ values (and also entanglement relaxation time, $\tau_{\mathrm{e}}$, values) from the monomer mean-square-displacement (MSD) results obtained from MD simulation of linear [70, 73, 142-144] and SCB PE chains $[72,74]$. For SCB melts, the tube diameters obtained from the simulations, whatever the method used, increase with SCB content, indicating that the melts of the most branched systems are less entangled. Results are in qualitative agreement with those obtained in experiments in which $d_{T}$ values are obtained using Equation (7) and independent measurements of $\mathrm{M}_{\mathrm{e}}$ (rheology) and $\left\langle\mathrm{R}_{\mathrm{g}}{ }^{2}\right\rangle / \mathrm{M}$ (SANS-derived chain dimensions), only available for HPB or EB copolymers [79]. On close inspection of the results, some striking differences emerge between the geometric or MD/MSD approaches and the experimental data, as can be observed in Figure 4. It should be noted that excellent agreement is observed between the results obtained by the MD/MSD method and the experimental data in the limited content range explored. Unfortunately, there are no results in the literature for simulations including higher SCB contents. Such results would be useful to test both geometric and dynamic approaches used to examine the entanglement features of polymeric melts. 
For the lower branching contents $\left(1-S_{c} \rightarrow 1\right)$, geometric methods yield higher values of $\mathrm{d}_{\mathrm{T}}$ than both the dynamic method and experiments (a comparison of results for linear PE is provided in Table 2). As can be observed, geometric algorithms give values of the tube diameter of around $4.0-4.3 \mathrm{~nm}$ for linear PE. In contrast, dynamic methods offer values around $3.2-3.6 \mathrm{~nm}$, which are closer to those reported for rheological experiments [79]. Recently, Hou [152] offered as an explanation for the differences observed in entanglement lengths of a given system using different approaches, a dimensionless pre-factor loss in deriving the relationship between $\mathrm{d}_{\mathrm{T}}$ and the segmental mean-squared-displacement cross-point at $\tau_{\mathrm{e}}$. However, the inversion of the trend shown in Figure 4 points to a complex relationship between the values obtained using the different methods and may suggest a complex dependence of the chemical structure of the entangled melt. The experimental results obtained for HPB by means of NSE are provided in Table 2, as this material is considered a model PE, despite containing considerable amounts of SCB. It can be clearly observed that the values of all entanglement features are notably higher than those obtained for linear PE.

Table 2. Simulated and experimental entanglement properties of linear PE

\begin{tabular}{|c|c|c|c|c|c|c|}
\hline Sample & Reference & Method & $\begin{array}{c}\mathbf{T} \\
(\mathrm{K}) \\
\end{array}$ & $\begin{array}{c}\mathbf{d}_{\mathbf{T}} \\
(\mathrm{nm})\end{array}$ & $\begin{array}{c}\mathbf{M}_{\mathbf{e}} \\
(\mathrm{g} / \mathrm{mol})\end{array}$ & $\begin{array}{c}\tau_{\mathrm{e}} \\
(\mathrm{ns})\end{array}$ \\
\hline $\mathrm{C}_{250}$ & [84] & PP mapping & 450 & 6.0 & 1,250 & - \\
\hline $\mathrm{C}_{300}$ & [92] & $\mathrm{Z} 1$ & 423 & 3.70 & 840 & - \\
\hline $\mathrm{C}_{350}$ & [76] & MSD/KHT & 450 & 3.20 & - & $1.5-2.8$ \\
\hline \multirow{2}{*}{$\mathrm{C}_{500}$} & \multirow{2}{*}{ [142] } & $\mathrm{Z1}$ & 450 & 4.25 & $1,180^{b}$ & - \\
\hline & & MSD/TraPPE & 450 & $3.26^{\mathrm{d}}$ & $690^{c}$ & $2.1-2.9$ \\
\hline $\mathrm{C}_{700}$ & [153] & Z1/MSD & 450 & 4.20 & 1,150 & 2.5 \\
\hline $\mathrm{C}_{1000}$ & [64] & $\mathrm{Z} 1$ & 450 & 4.09 & $1,230^{\mathrm{b}}$ & - \\
\hline $\mathrm{C}_{1000}$ & {$[70,72]$} & MSD/TraPPE & 450 & $3.33^{\mathrm{e}}$ & $790^{\mathrm{c}}$ & 2.3 \\
\hline \multirow{2}{*}{$\mathrm{C}_{1000}$} & \multirow{2}{*}{ [71] } & $\mathrm{Z} 1$ & 450 & 4.10 & $1,167^{\mathrm{C}}$ & - \\
\hline & & CReTA & 450 & 4.07 & $1,150^{\mathrm{c}}$ & - \\
\hline $\mathrm{C}_{1000}$ & {$[89,90]$} & CG-MD & 450 & 4.40 & $960^{\mathrm{a}}$ & 6.0 \\
\hline PE & [154] & Rheology theory mapped & 463 & - & $\begin{array}{c}860 \\
1,035\end{array}$ & $\begin{array}{c}1.45 \\
7.0\end{array}$ \\
\hline $\mathrm{PE}$ & [155] & Rheology theory mapped & 463 & - & 1,200 & 4.7 \\
\hline $\mathrm{PE}$ & [79] & Rheology/SANS & 413 & $\begin{array}{c}3.22^{\mathrm{c}} \\
3.59^{\mathrm{a}, \mathrm{c}}\end{array}$ & $\begin{array}{c}832 \\
1040^{\mathrm{a}}\end{array}$ & - \\
\hline $\mathrm{HPB}^{\mathrm{f}}$ & {$[156,157]$} & NSE & 509 & 4.8 & 2,000 & 5.0 \\
\hline $\mathrm{PE}$ & [158] & Experimental & 450 & - & - & $3.6_{-1.2}^{+9.3}$ \\
\hline
\end{tabular}

${ }^{\mathrm{a}} \mathrm{M}_{\mathrm{e}}=4 \rho \mathrm{RT} / 5 \mathrm{G}_{\mathrm{N}}{ }^{0}$; ${ }^{\mathrm{b}}$ From simulated $\mathrm{N}_{\mathrm{e}}$; ${ }^{\mathrm{c}}$ Equation $7 ;{ }^{\mathrm{d}}$ Obtained from MSD d $\mathrm{d}_{\mathrm{t}}=2\left[\mathrm{~g}_{1}{ }^{*}(\mathrm{t})\right]^{1 / 2}[142-144] ;{ }^{\mathrm{e}}$ Obtained from MSD $\mathrm{d}_{\mathrm{t}}=\left[3 \mathrm{~g}_{1}{ }^{*}(\mathrm{t})\right]^{1 / 2}$ [137]: $\mathrm{g}_{1}{ }^{*}(\mathrm{t})$ denotes the segmental MSD at the time $t=\tau_{e}$ where the slope of begins to change as segments leave the initial $t^{1 / 2}$ regime (in a log-log plot) and enter the $t^{1 / 4}$ regime. ${ }^{\mathrm{f}}$ Hydrogenated polybutadiene contains until a 1.9 ethyl branches per $100 \mathrm{C}$.

At this point, it should be noted that the value of $\mathrm{M}_{\mathrm{e}}$ strongly depends on the approach adopted for its calculation. Padding and Briels [159] already observed that all methods based on time-resolved quantities, such as the dynamic structure factor, $\mathrm{S}(\mathrm{q}, \mathrm{t})$ and the relaxation modulus, $\mathrm{G}(\mathrm{t})$, nearly give the same result. Such a value is around 1.5 higher than that obtained from the "plateau" modulus, $\mathrm{G}_{\mathrm{N}}{ }^{0}$, which is a time-integrated quantity.

The effect of more complex architectures (LCB or cyclic molecules) has been also studied but to a lesser extent and only from a dynamic point of view. Karayannis et al. found the same value of $\mathrm{d}_{\mathrm{T}}$ in linear and $\mathrm{H}$-shaped PE samples, around 2.9 and $3.3 \mathrm{~nm}$, respectively [76]. In the case of long ring PE, a broader crossover from unentangled to entangled dynamics with critical chain length for entanglements about three times larger 
than that for linear chains has been found $[59,60]$. However, the direct estimation of $d_{T}$ has not yet been reported.

\subsection{Dynamic properties: entanglement relaxation time and diffusion}

It is possible to extract information about the dynamics of systems from MD simulations. Segmental mean squared, $g_{1}(t)$, and centre of mass, $g_{3}(t)$, displacements can be directly obtained from the simulated atomistic trajectories as:

$$
\begin{gathered}
g_{1}(t)=\frac{1}{2 k+1} \sum_{n=\frac{N}{2}-k}^{\frac{N}{2}+k}<\left[r_{n}(t)-r_{n}(0)\right]^{2}> \\
g_{3}(t)=\frac{1}{N} \sum_{n=1}^{N}<\left[r_{c m, n}(t)-r_{c m, n}(0)\right]^{2}>
\end{gathered}
$$

,where $N$ stands for the total number of backbone atoms and $k$ is a parameter used to eliminate the effects of chain ends on the internal dynamics [84].

The reptation concept provides an elegant physical picture of the typical slowing down dynamics of polymers, clearly observed in the time evolution of $g_{1}(t)$ and $g_{3}(t)$. In this framework, the chain moves on a CG scale along its own contour. The main reason for this dynamics is that the surrounding molecules restrict the transverse motion of the chain. For short time scales, the motion of the monomers cannot be distinguished from that of the Rouse motion and $\mathrm{g}_{1}(\mathrm{t})$ scales as $\mathrm{t}^{1 / 2}$ and $\mathrm{g}_{3}(\mathrm{t})$ as $\mathrm{t}^{1}$. When the critical entanglement length for the monomer motion is reached, the segments start to feel the entanglements at a typical time given by $\tau_{\mathrm{e}} \sim \mathrm{N}_{\mathrm{e}}^{2}$. Above $\tau_{\mathrm{e}}, \mathrm{g}_{1}(\mathrm{t})$ scales as $\mathrm{t}^{1 / 4}$ and $\mathrm{g}_{3}(\mathrm{t})$ as $\mathrm{t}^{1 / 2}$. The chain then relaxes all along its contour and, at $\tau_{\mathrm{R}} \sim \mathrm{N}^{2}$, an overall diffusion is produced along the tube yielding a second $t^{1 / 2}$ regime for $g_{1}(t)$ and $t^{1}$ regime for $g_{3}(t)$. Finally, after a time $\tau_{\mathrm{d}} \sim \mathrm{N}^{3} / \mathrm{N}_{\mathrm{e}}$, the so-called disentanglement time, overall diffusion of the chain in space is observed, for which $\mathrm{g}_{1}(\mathrm{t})$ scales as $\mathrm{t}^{1}$.

Harmandaris et al. reported a clear transition from Rouse $\left(t^{1 / 2}\right)$ to reptation-like dynamics $\left(\mathrm{t}^{1 / 4}\right)$ in $\mathrm{g}_{1}(\mathrm{t})$ for $\mathrm{C}_{200}$ melts [84]. In addition, transitions at $\tau_{\mathrm{R}}$ and $\tau_{\mathrm{d}}$ were observed up to molecular lengths of $\mathrm{C}_{250}$. It is well known that crossovers are smooth, making it difficult to precisely identify the characteristic times for the different regimes. The exact corresponding values of the slopes predicted by the theory in the different zones were in no case clearly attained, most likely because overlap between the Rouse diffusion and tube constraints on the segmental dynamics is not sufficient for long polymer chains $\left(<\mathrm{C}_{500}\right)$. Better agreement with theoretical slopes, at least in the Rouse to reptation transition, has been observed in atomistic MD simulations (TraPPE-UA) of longer chains $\left(\mathrm{C}_{1000}\right)$ [70, 72-74]. Unfortunately in this latter case, the simulation time was shorter (up to $100 \mathrm{~ns}$ ), and thus the third $\left(\mathrm{t}^{1 / 2}\right)$ and fourth $\left(\mathrm{t}^{1}\right)$ regimes were not reached, though the results well matched those of prior simulations performed by Harmandaris et al. [84] and Karayannis and Mavrantzas [75, 76] using HMT-UA and KMT-UA FFs. In Figure 5A it is possible to observe the different regimes in atomistic simulations of $\mathrm{EB}$ and $\mathrm{EH}$ copolymers of different chain length and the same concentration of ethyl or butyl branches (1.9 branches every $100 \mathrm{C}$ atoms) for molecular lengths from $\mathrm{C}_{36}$ to $\mathrm{C}_{1000}$. Below $\mathrm{C}_{192}$, the characteristic Rouse behaviour is observed involving direct transition from $\mathrm{t}^{1 / 2}$ to $\mathrm{t}^{1}$ dependence. For $\mathrm{C} 192$ and longer chains, entanglement effects are observable as the development of $t^{1 / 4}$ and $t^{1 / 2}$ intermediate transitions. There are few experimental $\mathrm{g}_{1}(\mathrm{t})$ data for polyolefins to compare with those obtained in MD simulations. In Figure 5A, we show the only case found in the literature [72, 74]. The 
experimental evolution of $\mathrm{g}_{1}(\mathrm{t})$ was obtained from the extremely difficult incoherent NSE experiments conducted by Wischnewski et al. in the PEB2 sample [160]. These data measured at $\mathrm{T}=509 \mathrm{~K}$ are compared to those obtained by MD simulation of an $\mathrm{EH}$ copolymer $\left(\mathrm{C}_{1000}\right.$ with 1.9 butyl branches per $\left.100 \mathrm{C}\right)$ shifted from $\mathrm{T}=450 \mathrm{~K}$ using the corresponding $\mathrm{E}_{\mathrm{a}}=30 \mathrm{~kJ} / \mathrm{mol}$. The computed atomistic result adequately matches the experimental data.
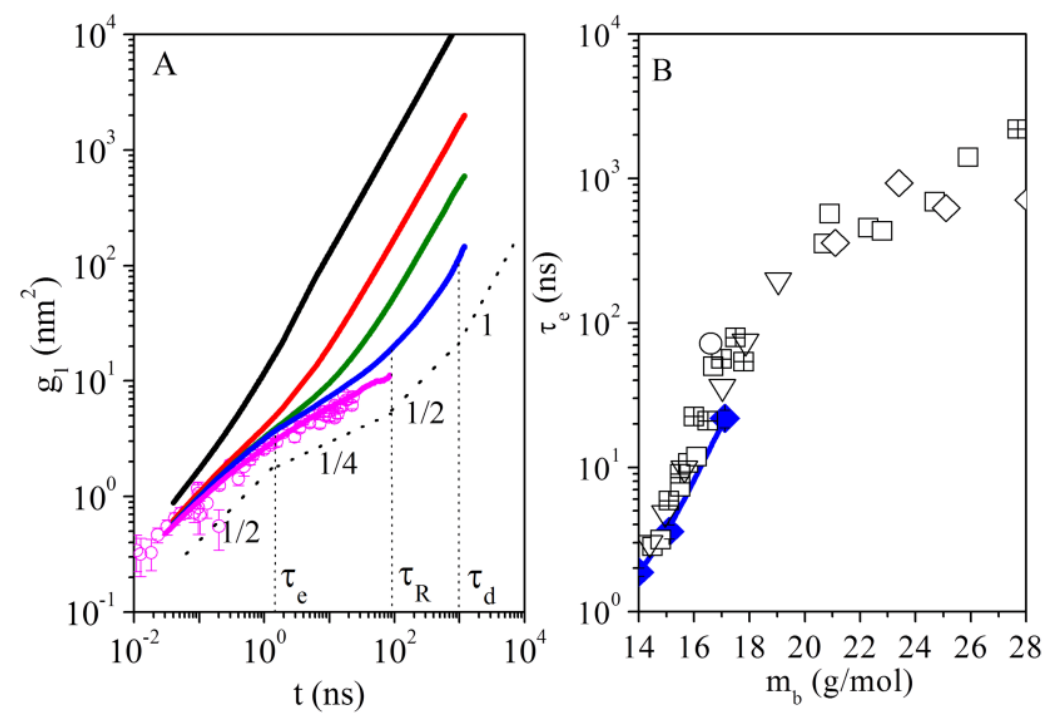

Figure 5: (A) Time evolution of mean-squared monomer displacement, $g_{1}(t)$, for simulated PEB2 and PEH2 samples at $509 \mathrm{~K}$ (solid lines). Included are the experimental data obtained for a PEB2 sample of $M_{\mathrm{w}}=190 \mathrm{~kg}$ $\mathrm{mol}^{-1}$ (symbols). Dashed lines indicate the Rouse, entanglement and diffusion regimes. Black, red, green, blue and pink lines correspond to PEB2_36, PEB2_106, PEB2_192, PEB2_377, and PEH2_1000, respectively [72, 74]. Adapted from J. Ramos, J.F. Vega, J. Martinez-Salazar. Soft Matter (2016) 12 (17), 3929-3936 with permission from The Royal Society of Chemistry. (B) Effect of SCB on entanglement relaxation time.

Experiments + tube models: $(\square) \mathrm{EB},(\boxplus) \mathrm{HPB},(\bigcirc) \mathrm{EP},(\diamond) \mathrm{EH}$ and $(\nabla)$ longer ethylene/ $\alpha$-olefins [132-136]. $(\diamond) \mathrm{MD} / \mathrm{TraPPE}-\mathrm{UA}$ simulations for EH copolymers [72, 74, 132, 133].

Karayannis and Mavrantzas also noted close agreement with the theoretical exponents in MD simulations of long, $\mathrm{H}$-shaped PE models (up to $\mathrm{C}_{500}$ ) at $\mathrm{T}=450 \mathrm{~K}$ [76]. These authors also estimated $\tau_{\mathrm{e}}$ and $\mathrm{d}_{\mathrm{T}}$ and found similar values of $\tau_{\mathrm{e}}(1.5-2.8 \mathrm{~ns})$ and $\mathrm{d}_{\mathrm{T}}\left(2.9-3.2 \mathrm{~nm}\right.$ ) for the linear and H-shaped PE models (up to $\mathrm{C}_{500}$ ). For the Rouse to reptation transition, agreement with the results obtained from MD simulations of a $\mathrm{C}_{1000}$ sample at the same temperature was good, giving values of $\tau_{\mathrm{e}}=2.32 \mathrm{~ns}$ and $\mathrm{d}_{\mathrm{T}}=$ $3.3 \mathrm{~nm}[70,72-74]$ using the Likhtman and McLeish methodology. Stephanou et al. reported values of $\tau_{\mathrm{e}}=2.1-2.9 \mathrm{~ns}$ and $\mathrm{d}_{\mathrm{T}}=3.2-3.3 \mathrm{~nm}$ for atomistic models of linear PE samples (C320-C500) using a similar procedure [142-144]. More recently, Sefiddashti et al. described $\tau_{\mathrm{e}}=2.47 \mathrm{~ns}$ for a $\mathrm{C}_{700}$ sample at $\mathrm{T}=450 \mathrm{~K}$ [153]. In Table 2 , the values obtained for $d_{t}$ and $\tau_{e}$ are listed in the case of linear PE, including experimental results obtained from mapping to tube theories of rheological data [154] and a recent experimental value for $\tau_{\mathrm{e}}$ obtained at high frequencies reported by Szanto et al. [158]. We should highlight the agreement between simulated and experimental values in the lower wing of the confidence interval of the experimental results. 
The impacts of SCB on diffusion regimes have also been addressed through computer simulations. The works of Ramos et al. [72, 74] identified a strong effect of $\mathrm{SCB}$ on $\mathrm{g}_{1}(\mathrm{t})$ for a given chain length. Simulated values of $\mathrm{g}_{1}(\mathrm{t})$ for different polymers $\left(\mathrm{C}_{1000}\right)$ with SCB contents of up to $55 \mathrm{SCB}$ every $1,000 \mathrm{C}$ atoms reveal a clear dependence of $\tau_{\mathrm{e}}$ and $\mathrm{d}_{\mathrm{T}}$ on SCB content. The changes produced in $\tau_{\mathrm{e}}$ involve variations in the Rouse monomer diffusion rate, $\mathrm{W}$, and consequently in the monomeric friction coefficient, $\zeta_{0}$. There is no definite explanation for the origin of these dependencies, but some authors have suggested a possible effect of the volume associated with statistical segment length on the dynamic processes [161]. The decrease in Rouse rate and subsequent increase in $\zeta_{0}$ as SCB rises suggests a reduction in the segmental motion probability [162], in agreement with lattice simulations of the relative motion and interactions of chain segments of model polyolefins [163].

A comparison with values obtained from rheological measurements is possible. Effectively, García-Franco et al. [134, 135] and Chen et al. [136] obtained $\tau_{\mathrm{e}}$ values for different model polyolefins including different single-site ethylene/ $\alpha$-olefins and HPB. This comparison is shown in Figure $5 \mathrm{~B}$ for a temperature of $\mathrm{T}=463 \mathrm{~K}$ and requires shifting of computed and experimental data using proper values of the flow activation energy of each material $[132,133,136]$. The results obtained are promising for values of $\mathrm{m}_{\mathrm{b}}$ up to $18 \mathrm{~g} / \mathrm{mol}$, and prompt future simulations involving higher SCB contents.

Only a few studies have extracted $\tau_{\mathrm{R}}$ and $\tau_{\mathrm{d}}$ from a limited number of atomistic models for linear [72, 74, 76, 142-144], H-shaped [76] and SCB PEs [72, 74]. These studies have detected higher $\tau_{\mathrm{R}}$ and $\tau_{\mathrm{d}}$ values for H-shaped and SCB PE samples than for their linear counterparts (same number of $\mathrm{C}$ atoms), indicating additional relaxation mechanisms of dangling arms and additional contributions of friction of the branch points.

Alternative comparisons with the literature can be made according to the dynamic melt structure factor, $\mathrm{S}(\mathrm{q}, \mathrm{t})$, or the centre of mass displacement, $\mathrm{g}_{3}(\mathrm{t})$, obtained from Neutron Spin Echo (NSE). The utility of performing experiments and simulations on well-characterized systems for the study of polymer dynamics was demonstrated by Smith et al. in the $\mathrm{C}_{100} \mathrm{n}$-alkane using both all explicit and UA simulations with the PYS FF [40, 164]. Good agreement was observed between experiments and simulations of the melt dynamic structure factor in the time window below 20 ps and contributions to polymer dynamics were attributed to the combination of torsional librations and torsional jumps. Results are also available for monodisperse PEB2 samples provided by Zamponi et al. [110]. Figure 6 compares results obtained for $\mathrm{g}_{3}(\mathrm{t})$ from MD simulations in atomistic PEB2 systems (TraPPE-UA) that match the PEB2 experimental samples for those NSE experiments conducted at $\mathrm{T}=509 \mathrm{~K}[72,74]$. Agreement was excellent, and it can be observed that for $\mathrm{t}<\tau_{\mathrm{e}}$, dynamics are subdiffusive and $\mathrm{g}_{3}(\mathrm{t}) \sim \mathrm{t}^{\mathrm{z}}$ where $\mathrm{z}<1$, in disagreement with the exponent predicted by the Rouse model for free diffusion $(\mathrm{z}=1)$. Atomistic simulations show a characteristic decrease in the time exponent as $\mathrm{N}$ increases, in accordance with the experiments. The inset in Figure 6 compares the results obtained by Ramos et al. [72, 74] and Guenza in simulations of short $n$-alkanes $\left(\mathrm{C}_{16}\right.$ to $\left.\mathrm{C}_{44}\right)$ in the temperature range $\mathrm{T}=298-400 \mathrm{~K}$ [165-167] and also with simulations in $\mathrm{C}_{1000}$ linear and SCB PE at $\mathrm{T}=450 \mathrm{~K}[69,71]$. It has been recently shown that CG models of polymer melts applied to linear PE preserve the structural features of the polymer chains and also capture the characteristic regimes of the reptation model in $\mathrm{g}_{1}(\mathrm{t})$ [89, 90, 92, 94-97]. However, these models are unable to capture the sub-diffusive regimes in $\mathrm{g}_{3}(\mathrm{t})$ reported in atomistic simulations, as they seem to follow Rouse dynamics. By examining $\mathrm{g}_{3}(\mathrm{t})$ from $\mathrm{CG}$ simulations with constraints, Padding and Briels noted a Rouse-like behaviour in unentangled systems but a 
subdiffusive exponent in entangled systems [89]. They assigned this subdiffusive exponent to uncrossability with respect to the surrounding chains. However, recent approaches using hybrid atomistic/CG multiscale models are able to restore the dynamics regime seen in the atomistic simulations as the atoms/beads ratio is increased [168].

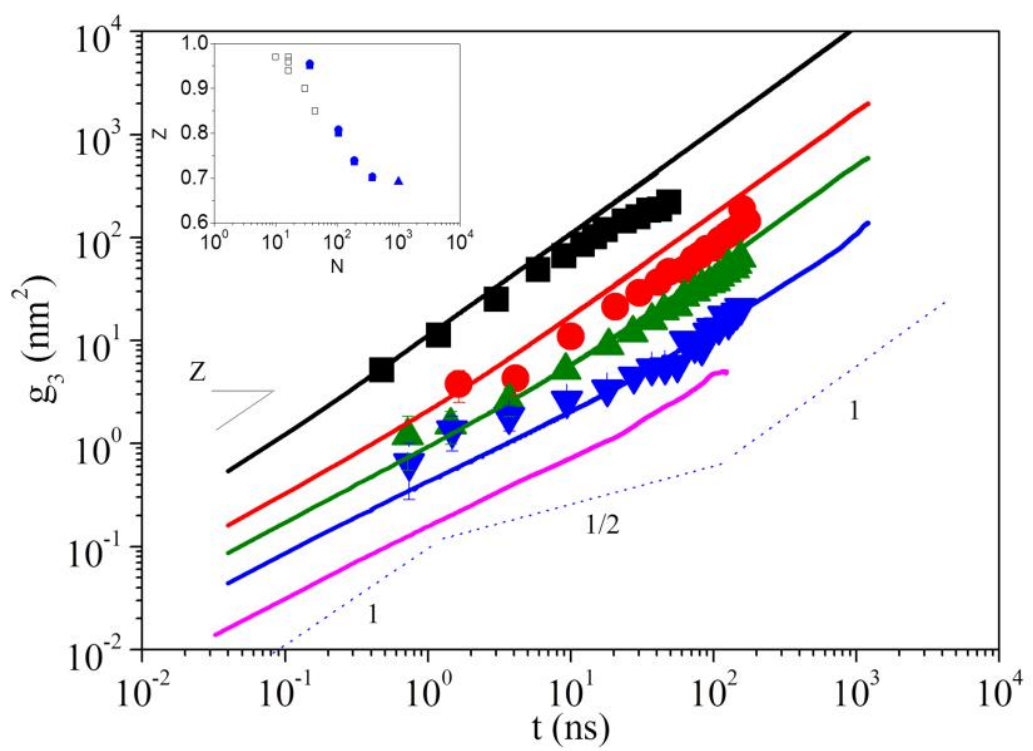

Figure 6: The centre of mass mean-square displacement, $\mathrm{g}_{3}(\mathrm{t})$, extracted from MD simulations for PEB2 and PEH2 systems (solid lines for $\mathrm{N}=36,106,192,377$ and 1000 from up to down) and compared to the experimental values at $\mathrm{q}=0.3 \mathrm{~nm}^{-1}$. Symbols: experimental data for $\mathrm{N}=36(\boldsymbol{\square}), 106(\boldsymbol{O}), 192(\boldsymbol{\Delta})$, and 377 ( $\boldsymbol{\nabla})$ from Zamponi et al. [110]. The inset shows the molecular length dependence of the slope $\mathrm{z}$, obtained from $\log \left[\mathrm{g}_{3}(\mathrm{t})\right]$ vs. $\log (\mathrm{t})$ over short times for $\mathrm{n}$-alkanes and different PE models. Open and filled symbols correspond, respectively, to the theoretical results for $(\square)$ n-alkanes [165-167] and for ( $\boldsymbol{\square})$ linear and ( $\mathbf{\Delta})$ branched PE [74]. Adapted with permission from J. Ramos, J.F. Vega, J. Martinez-Salazar Macromolecules, 48:2015, 5016-5027. Copyright 2015 American Chemical Society.

The trend observed in the $z$ exponent is quite similar irrespective of chemical monomer nature, but absolute values of $\mathrm{z}$ might be affected by both monomer type and temperature. It is worthwhile mentioning here that simulations predict a levelling off in the value of $z$ close to a limit around $0.69-0.70$. Guenza proposes a theoretical approach based on generalized Langevin equations, in which the inclusion of local intermolecular forces improves the description of global dynamics for both nonentangled and entangled systems [165-167]. This theoretical approach gives rise to nonuniversal scaling of the $z$ power law exponent that decreases as chain length increases. The model also predicts a levelling off in the number of correlated chains, which is the number of chains contained in the volume defined by two entanglement points as the entangled regime is approached. The results obtained in MD simulations of linear and SCB PE of different molecular lengths are in accordance with this theoretical approach. In this framework, the application of Guenza's theoretical model to different molecular architectures would be of great interest, as it is well known that both chain conformation and entanglement properties are strongly affected by the local chain architecture.

MD computer simulations of ring models based on chain lengths ranging from $\mathrm{C}_{24}$ to $\mathrm{C}_{400}$ have been described by Hur et al. [59, 60] (using PYS-UA) and Tsolou et al. [65] (using KMT-UA). The most notable results obtained from these simulations are that ring PE melts follow Rouse-like dynamics, even when chains are as long as $\mathrm{C}_{400}$. This length is greater than the characteristic crossover chain length marking the passage from Rouse to reptation dynamics of the corresponding linear PE melts. It is thus 
expected that the entanglement dynamics of PE rings in a melt, if this exists at all, will be seen better in higher molecular weight forms than in their linear counterparts. As rings cannot entangle in the classic sense, they are not expected to show reptation-like behaviour. Longer ring chains, up to $\mathrm{C}_{1500}$, show a transition from Rouse-type to entangled dynamics, as indicated by $\mathrm{g}_{1}(\mathrm{t})$. The $\mathrm{g}_{1}(\mathrm{t})$ transition from $\mathrm{t}^{0.5}$ to $\sim \mathrm{t}^{0.35}$ which is similar to entangled linear chains, has been observed at a value 3 times the corresponding $\mathrm{N}_{\mathrm{e}}$ of linear PE [59, 60, 65].

The long-term behaviour of $\mathrm{g}_{3}(\mathrm{t})$ obtained from MD simulations allows for direct determination of the self-diffusion coefficient, D. Most of the literature results correspond to linear (up to $\mathrm{C}_{1000}$ ) $[47,48,58,72,74,81]$ and ring (up to $\mathrm{C}_{1500}$ ) $[59,60$, 65] PE models, and more recently to PEB2 samples (up to $\mathrm{C}_{377}$ ) [72, 74]. CG models have served to assess PE chains up to $\mathrm{N}=2080$ [89, 90, 97]. For linear polymers, reptation theory predicts $D \sim N^{-2}$ for $N>N_{e}$, but experimentally the observed scaling is $N^{-2.3}$. This experimental behaviour is also observed in simulations for linear PE regardless of the FF used. Reduced values of $\mathrm{D}$, but with the same $\mathrm{N}$ dependence have been described for $\mathrm{H}$-shaped models of PE [75] and the effective friction assigned to curvilinear motion at the branch points, as proposed by McLeish and Larson [169]. The results obtained for ring PE systems are similar to those described for linear PE, but shifted to significantly faster diffusion as $\mathrm{N}$ increases. For the longest ring chains (up to $\mathrm{C}_{1500}$ ), a chain length dependence of diffusion coefficients $\mathrm{N}^{-1.9}$ approaches the corresponding value obtained for linear architectures. Notwithstanding, while direct evidence for entanglements has not been observed in long ring polymers, threading between the rings is suspected to be the main reason for the different dynamics of ring polymer diffusion [170]. In the case of SCB PE (PEB2), it is interesting to note that simulations performed on linear PE with the same polymerization degree as its analogue PEB2 give rise to lower values of $D$ at the same temperature. However, when results are plotted as a function of molecular weight, all simulations match the correlation obtained for both PEB2 and linear PEs [72, 74]. This finding has meant that we can consider PEB2 as a model for linear PE, even when 1.9 ethyl branches for every 100 carbon atoms still have measurable effects on both conformational features and chain dynamics.

\subsection{Rheological properties}

\subsubsection{Mapping onto Rouse and tube models}

The relaxation modulus response after a small perturbation, $G(t)$, can be expressed by the stress autocorrelation function according to the fluctuation-dissipation theorem. In MD computer simulations of chain models, $G(t)$ can be determined via equilibrium stress autocorrelations using the Green-Kubo relationship:

$$
G(t)=\left(V / k_{B} T\right)\left\langle\sigma_{\alpha \beta}(t) \sigma_{\alpha \beta}(0)\right\rangle
$$

,where $\sigma_{\alpha \beta}$ are the off-diagonal components (xy, $\mathrm{xz}$ and $\mathrm{yz}$ ) of the stress tensor.

It is possible to identify the different dynamic regimes in this viscoelastic function. In the Rouse regime, $\mathrm{t}<\tau_{\mathrm{e}}, \mathrm{G}(\mathrm{t}) \sim \mathrm{t}^{-1 / 2}$, while for longer times, $\tau_{\mathrm{e}}<\mathrm{t}<\tau_{\mathrm{d}}, \mathrm{G}(\mathrm{t})$ shows a "plateau" at $\mathrm{G}_{\mathrm{N}}{ }^{0} \sim 1 / \mathrm{M}_{\mathrm{e}}$. For $\mathrm{t}>\tau_{\mathrm{d}} \mathrm{G}(\mathrm{t})$ relaxes to zero after the chains have reached the diffusive regime. Calculations of $G(t)$ from atomistic models of n-alkanes based on Equation (9) have been reported by Harmandaris et al., but only for short times, i.e., less than 10 ps. For longer times, statistical noise in the calculated time 
autocorrelation function values were too large for meaningful results [171]. Padding and Briels applied the same methodology to $\mathrm{CG}$ linear PE models $\left(\mathrm{C}_{80}-\mathrm{C}_{800}\right)$ using their non-crossing constraint models and combining the statistical computation of $G(t)$ with the use of Rouse and tube models $[89,90]$. They successfully obtained combined results for $G(t)$, from which a value of $\mathrm{G}_{\mathrm{N}}{ }^{0}=2.4 \mathrm{MPa}$ for $\mathrm{C}_{800}$ sample was extracted.

Because of intense variation in off-diagonal components of the stress tensor in Equation (9), long configuration averaging is required to calculate $G(t)$. These limitations for the evaluation of $G(t)$, have prompted the direct mapping of computed properties obtained by MD simulations onto the Rouse and tube models. Accordingly, it has been possible to obtain interesting parameters such as the monomeric friction coefficient, $\zeta$, and the Newtonian viscosity, $\eta_{0}$, in both the unentangled $[47,48,58]$ and entangled regimes [84].

Harmandaris et al. actually determined crossover from the Rouse to the entangled melt regimes in linear PE using the computed values of the diffusion coefficient, $\mathrm{D}$, together with an estimated value of the tube diameter, $\mathrm{d}_{\mathrm{T}}=6.0 \mathrm{~nm}$ [84]. This high value of $\mathrm{d}_{\mathrm{T}}$ for linear PE can be justified by chain length, which is shorter than $\mathrm{C}_{250}$, and thus is affected by additional relaxation mechanisms besides reptation, i.e. contour length fluctuations (CLF) and constraint release (CR). This methodology has been also applied to $\mathrm{H}$-shaped [76] and ring PE models [65]. Obviously, H-shaped models show higher values of $\eta_{0}$ compared with their linear counterparts. These values parallel the increased values of $\tau_{R}$ and $\tau_{d}$ assigned to entangled dangling arms and additional contribution of friction of the branches, as indicated above and in agreement with general experimental observations in LCB PE samples [172] [163]. On the contrary, ring melts are characterized by smaller $\zeta$ and $\eta_{0}$ values than their linear analogues as a consequence of the faster dynamics exhibited by the ring chains as compared to linear ones. The $\eta_{0}$ value of linear PE chains increases sharply beyond approximately $\mathrm{C}_{156}$ as the crossover from Rouse to reptation dynamics occurs (see Figure 7 , blue solid circles). In contrast, the viscosity of the corresponding ring PE melts undergoes a smoother change with molecular weight, even for ring chains as long as $\mathrm{C}_{400}$, thus continuing to approximately follow the Rouse scaling. Ramos et al. [70, 73] directly mapped the double reptation theory, using the values of $M_{e}$ and $\tau_{e}$ obtained from $\mathrm{MC}$ and $\mathrm{MD}$ simulations in a $\mathrm{C}_{1000}$ linear sample (see Figure 7, blue solid squares). These authors were thus able to suitably describe the experimental values of $G(t)$ for different monodisperse and polydisperse linear and SCB PE samples. The double reptation approach considers that $G(t)$ evolves in time as $\mathrm{G}(\mathrm{t})=\mathrm{G}_{\mathrm{N}}{ }^{0}[\psi(\mathrm{t})]^{2}$, where $\psi(\mathrm{t})$ is the Doi's tube survival probability [125]:

$$
\psi(t, M)=\frac{8 G}{\pi^{2}} \sum_{p=o d d}^{\infty} \frac{e^{t p^{2} / \tau_{d}}}{p^{2}}
$$

If we include CLF's contribution, then:

$$
\begin{gathered}
G=1-\kappa\left(\frac{M_{e}}{M}\right)^{0.5} \\
\tau_{d}=3 \tau_{e}\left(\frac{M}{M_{e}}\right)^{3}\left[1-\kappa\left(\frac{M_{e}}{M}\right)^{0.5}\right]^{2}
\end{gathered}
$$


,where $\kappa=1.69$ as obtained by Likhtman and McLeish [137]. Remarkably, this mapping approach is able to assess the impacts of high molecular weight tails in the molecular weight distribution $[173,174]$, and also explain the declining values of $\eta_{0}$ as SCB increases, long discussed in the literature [132, 133]. Stephanou et al. [142-144] developed a dynamic mapping method based on the $\mathrm{Z} 1$ code to quantify stress relaxation in entangled polymer melts. Using this method, they also computed $\psi(\mathrm{t})$ describing the survival probability of PP segments directly from atomistic MD results. The methodology first maps long atomistic MD trajectories onto time trajectories of primitive chains, and then establishes primitive chain motion in terms of curvilinear diffusion in a tube-like region around the CG chain contour. In the calculations, the effective $\mathrm{d}_{\mathrm{T}}$ is independently determined by observing the effect of the tube constraints either on atomistic displacements or on the displacement of primitive chain segments orthogonal to the initial PP. The approach accounts for chain reptation longitudinally inside the constraining tube as well as for local transverse fluctuations driven by CLF and CR. This approach has been applied to different chain models, including linear PE $\left(\mathrm{C}_{250}-\mathrm{C}_{500}\right)$, giving rise also to a good description of $\eta_{0}$ values (see Figure 7 , green solid symbols).

Very efficient numerical methods based on CG models are starting to emerge to approach the direct statistical computation of $G(t)$ from MD [175]. These methodologies allow for direct determination of characteristic values of $\mathrm{G}_{\mathrm{N}}{ }^{0}$ and $\eta_{0}$, without further assumptions of the mechanisms underlying the relaxation process of the systems. Salerno et al. estimated $\mathrm{G}_{\mathrm{N}}{ }^{0}=2.1-2.2 \mathrm{MPa}$ for $\mathrm{CG}$ models of linear PE up to $\mathrm{C}_{1920}$ for different $\mathrm{CG}$ levels [94-96] while recently, Sgouros et al. examined the time evolution of $G(t)$ for $\mathrm{CG}$ models of linear PE up to $\mathrm{C}_{2080}$, reporting a $\mathrm{G}_{\mathrm{N}}{ }^{0}$ value of 2.0 $\mathrm{MPa}$ [97]. In both studies, the computed values of $\mathrm{G}_{\mathrm{N}}{ }^{0}$ are very close to the reported experimental range $\mathrm{G}_{\mathrm{N}}{ }^{0}=2.17 \pm 0.23 \mathrm{MPa}[70,73,158]$. From these simulations $\eta_{0}$ can be calculated as:

$$
\eta_{0}=\int_{0}^{\infty} G(t) d t
$$

By applying Equation (13) to the results obtained from these CG models (see Figure 7 , open blue symbols) we can determine the corresponding values of $\eta_{0}$, and then compare results with existing experimental values or those obtained by mapping methods. For all cases, agreement with experimental results is excellent, describing quite well the crossover between Rouse and entangled regimes up to molecular weights of around $30 \mathrm{~kg} / \mathrm{mol}$. Notwithstanding, crucial information may be lost when CG models or simple analytic theories are used [175, 176]. Masubuchi et al. recently reported atomistic MD simulations of linear PE chains using TraPPE-UA FF [176]. These authors showed that the presence or absence of entanglements affects Rouse regime duration, being shorter in the more entangled systems. This effect cannot be captured by CG models. Moreover, theories based on Gaussian statistics ignore manybody effects, which can be described by long MD atomistic simulations. 


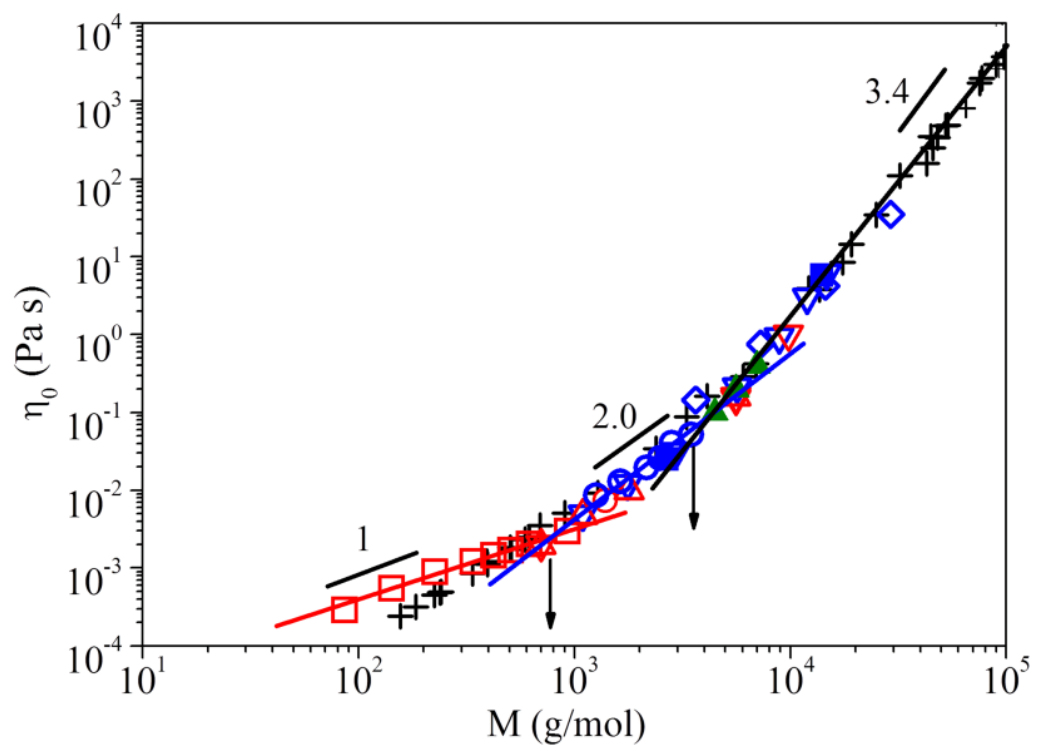

Figure 7: (十) Experimental values at $\mathrm{T}=450 \mathrm{~K}$ (see references in [171]). Mapped values: ( $\bigcirc$ ) Mapping onto Rouse and tube models [47, 48, 58, 84]; ( $)$ Mapping onto the double reptation model [61, 64] [70, 73]; ( $\boldsymbol{\Delta})$ Mapping values onto a dual constraint model [142-144]. Green-Kubo relationship: $(\nabla)$ CG-PE $[89,90] ;(\diamond)$ CG-PE [97]. NEMD simulations: $(\square)[81] ;(\bigcirc)[177,178] ;(\triangle)[179-190] ;(\nabla)[153,191]$

\subsubsection{Non-equilibrium molecular dynamics}

The rheological properties of simulated systems can also be assessed through nonequilibrium molecular dynamics (NEMD). Direct NEMD simulations have been promising as dynamics can be tracked over a window of time scales within the range of experimental conditions. This kind of simulation allows access to very interesting properties like the dependence of viscosity on shear and elongation rates (or stresses), normal stress coefficients, and even visualization of the molecular conformational changes induced by flow.

In the literature, calculations of $\eta_{0}$ from NEMD in atomistic models have been reported by Mondello et al. [81] but only for short alkanes $\left(<\mathrm{C}_{66}\right)$ at $\mathrm{T}=448 \mathrm{~K}$ and constant density $\left(\rho=0.766 \mathrm{~g} / \mathrm{cm}^{3}\right)$. Moore et al. [177, 178] examined linear $\left(\mathrm{n}-\mathrm{C}_{100} \mathrm{H}_{202}\right)$ and branched (squalene, $\mathrm{C}_{30} \mathrm{H}_{62}$ ) alkanes and found that additional branches lead to significantly increased viscosity. They also observed transition of viscosity from Newtonian to shear thinning, and this was correlated with the alignment of molecules with flow and with their rotational relaxation time. In their CG models of linear PE with chain lengths varying from $\mathrm{C}_{80}$ to $\mathrm{C}_{800}$, Padding and Briels [192] used NEMD simulations in simple shear (planar Couette flow) and predicted a transient overshoot for both shear stress and the normal stress difference at high shear rates, and also stronger shear thinning as molecular weight increased, in good agreement with experiments. Baig et al. have extensively worked on linear PE systems from $\mathrm{C}_{10}$ to $\mathrm{C}_{400}$ through NEMD simulations in both elongational [179-181] and shear flow [182-184]. They also analysed H-shaped PE [185] and more recently SCB PE [186-188]. Their intriguing results and conclusions have revealed: $(i)$ that segmental orientation is the primary molecular mechanism leading to stress overshoot in transient rheological properties in linear PE atomistic models; (ii) similar shear thinning in LCB (H-shaped) $\mathrm{PE}$ and linear PE, but stronger tension-thickening, as experimentally observed in LCB polyolefins [172]; and (iii) a fast random Brownian kinetics inherent to SCB leading to a lesser degree of structural deformation, to reduced shear-thinning, and to less elastic stress as compared with their linear counterparts. It should be noted that these kinds of simulation are being applied to confined linear PE systems [189] and to the problem of 
interfacial slip [190] which generally occurs in polymer systems and specifically in PE processing, giving rise to noteworthy distortion regimes [193-198]. Edwards et al. [153, 191] have recently extended this type of NEMD simulation to a $C_{700}$ linear PE system. We should mention that they have tested different tube models to explain the non-linear behaviour of entangled PE atomistic systems subjected to shear flow. They showed not only an additional physical phenomenon that needs to be incorporated into rheological models (periodic rotation and retraction cycles under strong steady shear flow), but also that tube orientation tensor, tube stretch, entanglement number and relaxation time changes under flow are not well implemented in quantitative prediction theories.

In all these works it is possible to directly extract the values of $\eta_{0}$ for the different systems at a low enough shear rate. Results (see Figure 7, red open symbols) have been compared with those arising from the mapping methodologies and from the GreenKubo relationship. Results are again excellent, well describing the experimental trend from molecular weights of 0.1 to $30 \mathrm{~kg} / \mathrm{mol}$. Noteworthy, the results obtained from NEMD by Mondello et al. [81] in the $\mathrm{M}_{\mathrm{w}}$ range below 1,000 $\mathrm{g} / \mathrm{mol}$ recover the Rouse model prediction. These results are inconsistent with experimental values in this region, as simulations were performed at constant density, $\rho$. A large increase is produced in $\rho$ in the unentangled region (see Section 2.1.), which may cause faster than Rouse scaling of the viscosity. Above 1,000 g/mol, a transition region with a higher slope (around 1.8) appears up to the critical molecular $\mathbf{M}_{\mathrm{c}}$ of around $3,500 \mathrm{~g} / \mathrm{mol}$, which is quite well described by those obtained by the mapping methodologies and the CG model of Padding and Briels. The CG simulations performed by Padding and Briels [89, 90] and Sgouros et al. [97] perfectly match the experimental results in the entangle regime, in which the slope takes on the well-known value of 3.4.

\section{Thermal transitions: glass transition and crystallization}

\subsection{Glass transition temperature of semicrystalline polyethylene}

\subsubsection{Experimental approach}

The issue of semicrystalline polymers

As with most semicrystalline polymers, PE has an amorphous fraction whose proportion depends on the molecular architecture. The amorphous fraction in all polymers shows a glass transition. However, due to the generally high crystallinity of $\mathrm{PE}$, the issue of its $\mathrm{T}_{\mathrm{g}}$ is difficult to resolve as its nature is strongly influenced by its occurrence in the crystalline surroundings. Indeed, amorphous segments are linked to the crystalline lamellae, restricting their movements. In the following subsections, we will try to explain our vision of the current state of the $T_{g}$ of PE. To this end, we first briefly describe the mechanical relaxation spectrum of PE.

\section{The mechanical relaxation spectrum of $P E$}

In general, the classification of the dynamic transitions of PE described some years ago by Poplin et al. is still widely accepted [199]. According to these authors, $\gamma$ relaxation is usually detected in the range -150 to $-120^{\circ} \mathrm{C}(123-153 \mathrm{~K}) ; \beta$ relaxation in the range -30 to $10^{\circ} \mathrm{C}(243-283 \mathrm{~K})$; and $\alpha$ relaxation in the range 30 to $120^{\circ} \mathrm{C}(303-$ $393 \mathrm{~K}$ ). However, the molecular mechanisms driving these transitions have been a long standing matter of debate. Comprehensive literature reviews by Boyd have described the experimental evidence and molecular interpretation of the characteristic relaxations of synthetic polymers [200-202]. 


\section{1. $\gamma$ relaxation}

Relaxation appearing at the lowest temperatures $(\gamma)$ is commonly small in magnitude. Some authors initially suggested that this relaxation arose from motions of disordered chains at the surface of the crystals [203], from movements of the defects in the crystalline regions [204], and from motions associated with both the crystalline and amorphous phases [205, 206]. However, Crissman demonstrated that this relaxation arises only from the amorphous phase since polycrystalline long-chain paraffins showed no $\gamma$ relaxation at all [207]. In the past, some authors have also assigned this transition to the characteristic cooperative segmental motion related to the glass transition temperature [208]. Others suggested that the low temperature $\gamma$ transition is related to local relaxation processes attributed to conformational changes involving sequences of a few bonds (crankshaft motions) in amorphous chain segments [202, 209, 210]. To conclude this short overview, it presently seems that this relaxation is not directly connected to the $\mathrm{T}_{\mathrm{g}}$.

\section{2. $\alpha$ relaxation}

The high temperature $\alpha$ (main) relaxation in ethylene-based polymers has been associated with the crystalline phase, since the magnitude of this relaxation usually increases with crystallinity. This relaxation has been assigned to crystal stem translation mechanisms [202] and reorientation motions of the chain units within the crystals [211213]. In some cases, two overlapping relaxations have been reported in this region, designated as $\alpha$ ' and $\alpha$ ' in order of increasing temperature: $\alpha$ ' is generally attributed to interlamellar slip/shear [214, 215] or segmental intra-lamellar deformation [216]; while $\alpha$ " is attributed to the uniform shear deformation of the lamellae because of the rotation and translation movements of chains along the c-axis $[215,216]$. Finally, Danch and coworkers [217] have considered the $\alpha$ relaxation in PE to take place in the interfacial regions of the semi-crystalline structure. The lamellae strongly disturb and stretch the chains within these regions. Thus, the chain constituting the bulk amorphous phase and the interphase must show different properties and mechanical behaviour.

\section{3. $\beta$ relaxation}

$\beta$ relaxation is almost negligible in conventional linear semicrystalline $\mathrm{PE}$, but gains significance in side branched polyolefins showing a moderate to low degree of crystallinity [206, 208]. This transition has been linked to various molecular mechanisms: diffusion movements of amorphous chain segments containing branch points [206], segmental motions occurring within the amorphous-crystal interfacial regions [199, 218], and cooperative motions of the amorphous phase related to the glass transition temperature [219].

Since the 1980s, considerable attention has been paid to the existence of a third intermediate phase in semicrystalline polymers forming an interfacial layer between crystalline and amorphous material. There is experimental support for this additional component, an interfacial region with intermediate order between crystalline and amorphous phases, based on proton nuclear magnetic resonance (NMR) [220, 221], Raman spectroscopy [222] and wide angle X-ray diffraction (WAXD) [223]. However, some recent works have recovered Boyer's idea (early 70s) about the coexistence of two miscellaneous amorphous fractions in semicrystalline polymers [224], a picture also supported by Cheng and co-workers in the late 80s [225]. These studies distinguish between a mobile or unconstrained amorphous fraction, and a rigid or constrained amorphous fraction. It is also noteworthy that micromechanical modelling has been 
widely used to estimate the physical properties of these semicrystalline materials through both 2- and 3-phase microstructural models. In general, 3-phase approaches give better predictions of experimental behaviour [226]. To tackle this problem, we undertook a systematic study using a series of ethylene/hexane copolymers of increasing 1-hexene contents [124]. The copolymers were structurally regular high molecular weight metallocene single-site ethylene/1-hexene random copolymers with narrow molecular weight distributions (see reference for more details).

\section{The role of the interphase}

The data listed in Table 3 [124] illustrate the variations with crystallinity (or branching content) of the characteristic $T_{g}$ and $T_{\beta}$ values obtained from DSC and DMTA experiments, respectively. In addition, the dependence of $\Delta \mathrm{C}_{\mathrm{p}}$ values from DSC measurements is also observed. Moreover, the constraining effect of crystals over the amorphous chains causes not only a reduction in chain flexibility but also provokes a decrease in intermolecular atomic distances. Thus fundamentally, the increase in $T_{g}$ with crystallinity is related to a higher local density and reduced segmental mobility in the amorphous phase produced by constraints between the amorphous segments and the crystal surfaces.

Table 3. Physical properties of ethylene/1-hexene copolymers

\begin{tabular}{|c|c|c|c|c|}
\hline Material* & \% mol 1-hexene & $\mathbf{T}_{\mathbf{g}}(\mathbf{K})$ & $\Delta \mathbf{C p}\left(\mathbf{J ~ g}^{-\mathbf{1}} \mathbf{K}^{-\mathbf{1}}\right)$ & $\mathbf{T}_{\boldsymbol{\beta}}(\mathbf{K})$ \\
\hline EH15 & 3.2 & 236.9 & 0.12 & 241.0 \\
\hline EH23 & 5.1 & 227.6 & 0.24 & 231.3 \\
\hline EH30 & 6.7 & 220.3 & 0.32 & 226.5 \\
\hline EH35 & 8.3 & 219.2 & 0.37 & 221.6 \\
\hline EH42 & 10.1 & 214.6 & 0.48 & 217.0 \\
\hline
\end{tabular}

*The notation indicates the number of SCB (butyl branches) every 1,000 C atoms

These results do support a picture of two mobile and rigid amorphous phases, at least in the materials studied here, as the amorphous phase transition detected by DSC, WAXD and DMTA is actually made up of the most flexible chains in the copolymers, but with a different degree of mobility depending upon crystallinity.

Simha and Boyer postulated an approximate empirical generalization for the relationship between $\mathrm{T}_{\mathrm{g}}$ and $\Delta \mathrm{C}_{\mathrm{p}}$ [227]:

$$
\Delta C_{p} T_{g}=K
$$

,with $\mathrm{K}$ lying within the range of values 80 and $150 \mathrm{~J} \cdot \mathrm{g}^{-1}$ with $\mathrm{T}_{\mathrm{g}}$ in Kelvin for all amorphous polymers. We used the Simha-Boyer value of $\Delta \mathrm{C}_{\mathrm{p}}=0.75 \mathrm{~J} \cdot \mathrm{g}^{-1}{ }^{\circ} \mathrm{C}^{-1}$ obtained from Equation (14) considering $\mathrm{T}_{\mathrm{g}}=-78{ }^{\circ} \mathrm{C}(195 \mathrm{~K})$ for amorphous PE. In conclusion, $\mathrm{T}_{\mathrm{g}}$ ( or $\mathrm{T}_{\beta}$ ) values increase as crystallinity does. This behaviour is associated with reduced segmental mobility and an increased density of the amorphous phase as the degree of crystallinity increases. This contention is supported by the fact that the average molecular distance in the amorphous regions, as determined by X-ray scattering, decreases with increasing crystallinity. A comparison with results from the literature indicates that the free volume does not play a significant role in the $T_{g}$ values 
measured in these samples. This picture supports the idea that the whole amorphous phase is actually restricted by the crystals.

Analysis of $\Delta \mathrm{C}_{\mathrm{p}}$ values at $\mathrm{T}_{\mathrm{g}}$ suggests that there exists a fraction of chains restrained by the presence of the crystals that does not contribute to the heat capacity change produced at the glass transition temperature. This fraction of the material has a higher rigidity than the restricted amorphous phase, and should correspond to an interfacial region between amorphous and crystalline. The amount of this interphase is shown to be comparable to that of the crystalline regions, and is found to increase as the branching content decreases. The interphase cannot be independently distinguished from the amorphous or crystalline phases during heating above $\mathrm{T}_{\mathrm{g}}$ by means of DSC or WAXD, but it may contribute to the mechanical response of the material, as revealed by DMTA experiments [124]. If we plot $\mathrm{T}_{\mathrm{g}}$ as a function of pure amorphous content, we obtain an extrapolated value for $\mathrm{T}_{\mathrm{g}}$ of approximately $-76^{\circ} \mathrm{C}(197 \mathrm{~K})$ for a pure amorphous PE (see Figure 8).

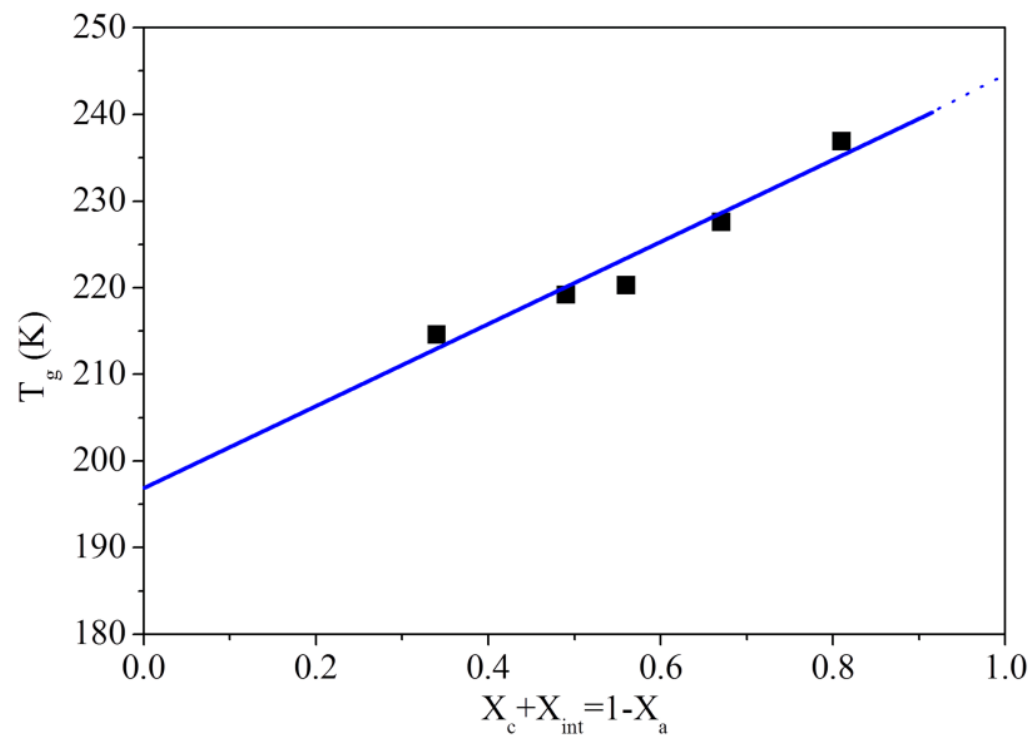

Figure 8. Glass transition temperature, $\mathrm{T}_{\mathrm{g}}$, versus crystal core and interfacial content, $1-\mathrm{X}_{\mathrm{a}}$. The line represents the linear extrapolation to $100 \%$ amorphous content

\subsubsection{Computer simulation approach}

In one of our recent studies, we were able to determine the $\mathrm{T}_{\mathrm{g}}$ value of PE. For this purpose, an equilibrated $\mathrm{C}_{192}$ melt system was cooled down at different cooling rates, $\Gamma$ $=1$ to $2,000 \mathrm{~K} \cdot \mathrm{ns}^{-1}$ (high enough to prevent polymer crystallization). Then, the specific volume as a function of temperature was measured using two different FFs. A change of the slope of the curves, i.e. the thermal expansion coefficient, indicates the apparent $\mathrm{T}_{\mathrm{g}}$, which is known to be dependent on the cooling rate [228]. The high cooling rates used in simulations generally lead to a higher estimate of $\mathrm{T}_{\mathrm{g}}$ compared to experiments.

The plot of apparent $T_{g}$ versus cooling rate is shown in Figure 9 for the systems studied. The figure reveals that $\mathrm{T}_{\mathrm{g}}$ decreases nonlinearly with the logarithm of the cooling rate, $\Gamma$, in both cases; this having been already observed in the literature both in experiments [228] and simulations [229-232]. In general, it was shown that the dependency resulted in a non-linear equation, adopting the form:

$$
T_{g}=T_{g}^{0}+\frac{A}{\ln (B \Gamma)}
$$


This expression can be extracted from the assumption of a Vogel-Fulcher dependence of relaxation times. The application of Equation (15) to experimental data provides the value of $\mathrm{T}_{\mathrm{g}}{ }^{0}$ for the system under study. Similar extrapolation procedures have been also applied in other computer simulations. [229-232].

The application of Equation (15) to the results in Figure 9 results in an estimated $\mathrm{T}_{\mathrm{g}}{ }^{0}=187.0 \mathrm{~K}$ and $214.1 \mathrm{~K}$ for TraPPE-UA and PYS FFs, respectively [73]. This last value is very close to those reported by Yi et al. using also PYS FFs of 215.2 and 223.0 $\mathrm{K}$ for a $\mathrm{C}_{150}$ and $\mathrm{C}_{1000}$ polymer, respectively [233]. Further, values of $\mathrm{T}_{\mathrm{g}}$ of $195.0 \mathrm{~K}$ [234] and $200.0 \mathrm{~K}$ [235] have been reported for $\mathrm{C}_{100}$ and $\mathrm{C}_{300}$ polymer models using explicit FFs, respectively. The values of $\mathrm{A}$ and $\mathrm{B}$ in Equation (15) are nearly the same for the two FFs, $A=368.7 \mathrm{~K}$ and $\mathrm{B}=0.023 \mathrm{~ns} \cdot \mathrm{K}^{-1}$. In fact it is possible to obtain a master curve from the two simulation sets by simply plotting $\mathrm{T}_{\mathrm{g}}-\mathrm{T}_{\mathrm{g}}{ }^{0}$ versus $\Gamma$, as may be observed in the inset to Figure 9. It should be noted that $\mathrm{T}_{\mathrm{g}}$ in polymers depends on factors such as chain stiffness, chain bulkiness (free volume) and the strength of polymer chain-chain interactions. All of these features are depicted by the specific FF used in the simulation.

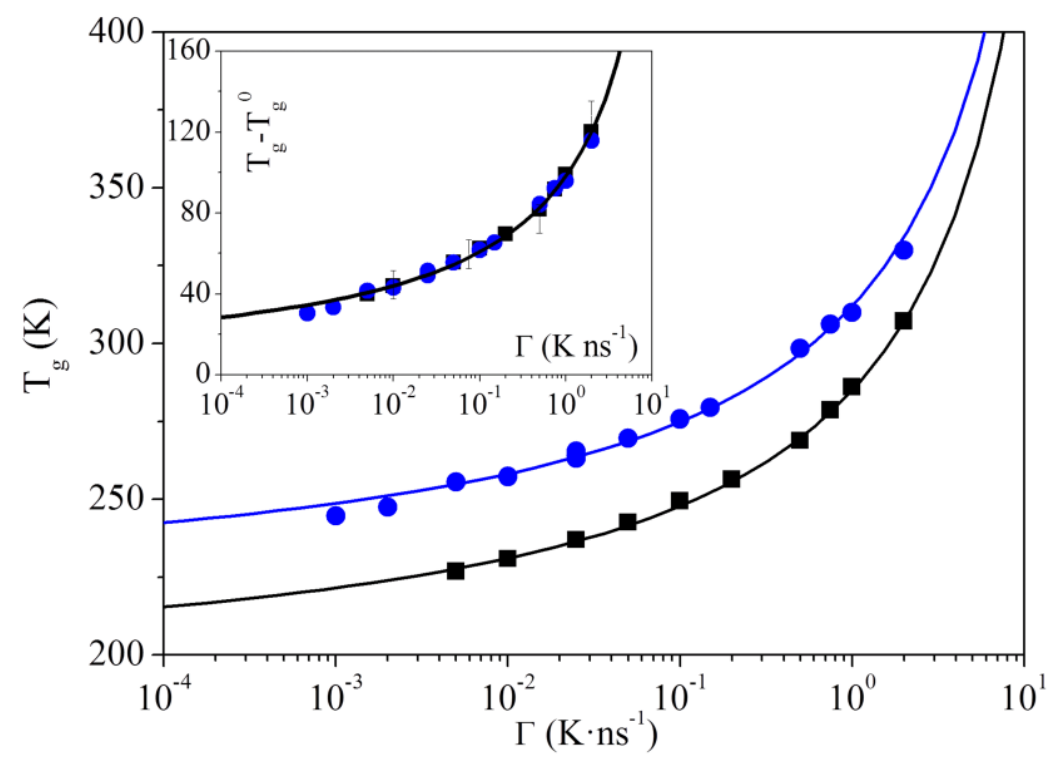

Figure 9. Apparent glass transition temperature versus cooling rate for the atomistic model $\mathrm{C}_{192}$ obtained from MD simulations using (O) PYS-UA and ( $\mathbf{a})$ TraPPE-UA force fields. The inset shows the master curve obtained by only shifting the data horizontally using the extrapolated glass transition, $\mathrm{T}_{\mathrm{g}}{ }^{0}$ (see text). The lines represent the fit to Equation (15) [73]. Reprinted with permission from J. Ramos, J.F. Vega, J. Martinez-Salazar. Macromolecules, 48:2015, 5016-5027. Copyright 2015 American Chemical Society.

It should be mentioned here that a recent theory is also able to predict the values of $\mathrm{T}_{\mathrm{g}}$ for different polyolefins [236]. In fact, this theory predicts a value of $\mathrm{T}_{\mathrm{g}}=196 \mathrm{~K}$ for LPE and also a so-called "internal plasticization" phase wherein fragility decreases as the number of flexible side group units increases.

The effect of SCB has only been recently explored but in hyperbranched dendritic PE models using MD simulations with TraPPE-UA FF [237]. The expected decrease of $\mathrm{T}_{\mathrm{g}}$ with branching content is obtained in agreement with parallel experimental results.

\subsection{The semicrystalline structure of polyethylene}




\subsubsection{The orthorhombic unit cell}

Density functional theory (DFT) has proved successful at accurately describing the electronic structure [238], crystalline structure and mechanical properties of crystalline PE [239]. DFT calculations on PE unit cell parameters lead to $a=6.7-6.8 \AA$ and $b=$ 4.5 - $4.6 \AA$ [240], much lower than those obtained in the experiments for bulk semicrystalline PE and single crystals [122, 241-244] likely due to overestimated intermolecular bond strengths. From all the calculated results of the above contributions, it can be deduced that mechanical properties, bulk modulus, elastic constants and Young's moduli are significantly larger than the experimental values, but consistent with inelastic neutron scattering spectra. Barrera et al. reported that these differences may be explained by the fact that we are comparing the properties of real semicrystalline materials, which have a relatively low crystallinity, with those of $100 \%$ crystalline models [245].

The introduction of empirical terms to account for dispersion (or van der Waals) interactions (see Section 4.1) has indeed improved results [246-248]. However, in weakly coupled systems such as polymers, the proper inclusion of VdW interactions is challenging [249, 250]. This measure, besides a stabilized crystal structure, gives rise also to values of the calculated lattice parameters $(a=7.30 \AA$ and $b=5.22 \AA)$ and bulk elastic modulus $\left(\mathrm{E}_{\mathrm{c}}=7.8 \mathrm{GPa}\right)$ that are in better agreement with experimental data than prior estimations without empirical input [248]. It should be noted that Román-Pérez et al. [251] and $\mathrm{Wu}$ et al. [252] later described a reformulation and a simplified implementation of this scheme. Further recent refinements and improvements by other authors have been reviewed in [239].

In addition, Martonak et al. applied a MC algorithm based on FF implementing global moves on the chains in addition to the local moves on the atoms to simulate crystalline linear PE. These authors obtained fairly accurate results for cell parameters, elastic constants and their temperature dependences using the FF developed by Sorensen et al. in the late 80s [253]. Mavrantzas et al. performed MD simulations on paraffins and infinite chain PE using COMPASS FF [254]. Their results were in good agreement with orthorhombic lattice parameters, the IR spectrum of crystalline PE and their dependence on temperature. [255] also conducted MD simulations using the all atom (explicit) FF of Smith and Yoon [39], also for linear PE. Since experimentally it can be clearly observed that lattice parameters depend on the disorder level in the crystals [121-123], these authors compared three different computer models considering different degrees of disorder, i.e. the infinite chain model, finite chain model and jogged chain model. This latter approach attempted to reproduce the effects of defects and chain folds by allowing a finite chain to pass through the simulation box more than once. The results of this work indicate that for the FF chosen, the jogged chain model is better suited to simulation of chain-folded PE crystals than the more ordered models, fairly well reproducing the lattice parameters and their temperature dependences. It should be noted here that quite similar results for the temperature dependences of linear PE lattice parameters can be obtained using quasi-harmonic lattice dynamics coupled to molecular mechanics FFs [256, 257]. 


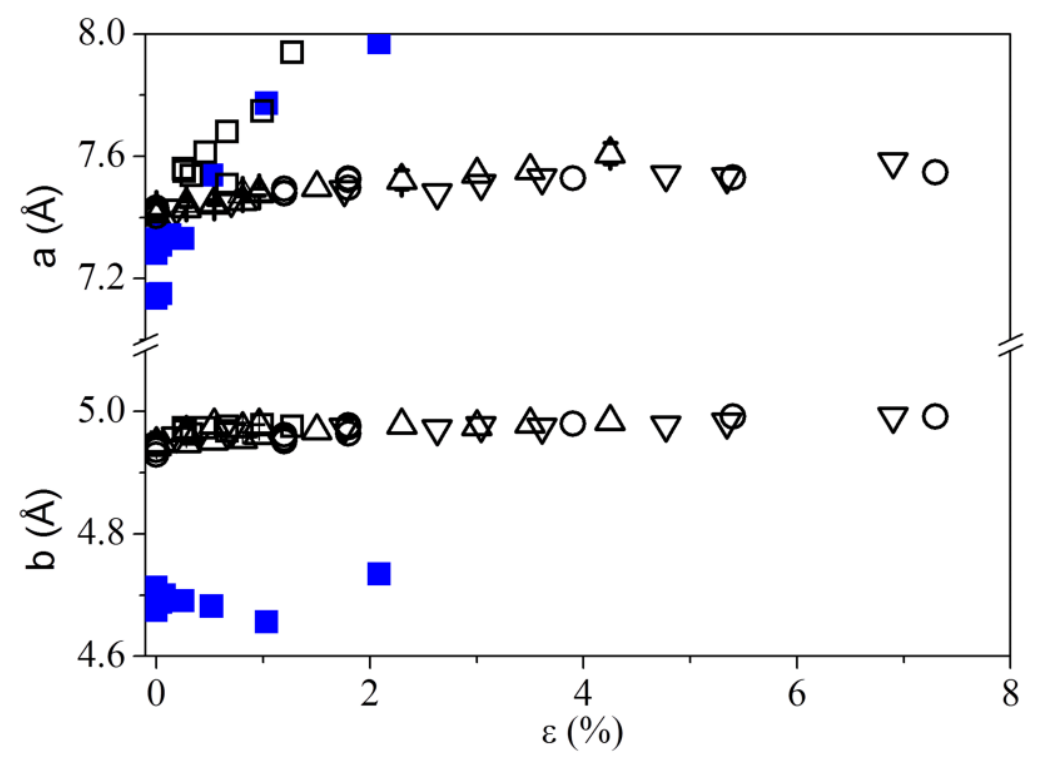

Figure 10: Orthorhombic lattice parameter variations produced with SCB in PE. Open symbols: experimental lattice parameters: $(\square)$ EP copolymers, Swan [242], (O) EB copolymers, Crist et al. [258], $(\triangle)$ branched PEs, Martínez-Salazar et al. [121-123], ( $\nabla)$ EH copolymers, Otegui et al. [243] and EH single-crystals, Vega et al. [244, 259]. Closed symbols represent computer simulations of lattice parameters including branching [255]

Less studied through computer simulations have been the impacts of SCB on orthorhombic lattice parameters. This has been a topic of much debate. On the basis of equilibrium thermodynamics, for instance, it could be argued that branches should be rejected from the crystalline regions during crystallization, as they represent a type of defect that will increase the internal energy of the crystal. However, the kinetics of crystallization is such that it is likely that some of the branches would be trapped within the crystals, suggesting some degree of partial segregation. Experimentally, it is accepted that methyl branches may be included in the crystals, and that these give rise to increased values of $a$ and $b$ due to a distorted lattice. A comparison of the classical values of Swan [242] for ethylene/propylene with those of the higher copolymers, ethyl [260], butyl [244] and longer branches [121-123] is provided in Figure 10. It may be clearly observed that the methyl branched copolymers show higher expansion of $a$ values than longer branched systems. The MD simulations conducted by Phillips and Hana [255] in orthorhombic cells including the branches in the lattice match the experimental results. The simulated $b$ parameter is always too low and attributed to errors in the $\mathrm{C}-\mathrm{H}$ bond length affecting the side-by-side packing density of the chains.

Recent DFT studies have also examined the Raman and infrared (IR) spectra of linear and branched PEs. The Raman crystallinity band appearing in linear PEs at $1415 \mathrm{~cm}^{-1}$ is thought to arise from factor group splitting, whereby the two PE chains packed in the orthorhombic unit cell cause the $\mathrm{CH}_{2}$ bending vibrational mode to split, with a second component appearing at $1440 \mathrm{~cm}^{-1}$ [261]. Changes in the crystalline phase, i.e. in the unit cell parameters, for example, as a result of temperature changes or the inclusion of SCB, could affect this phenomenon resulting in changes in interchain energy interactions [262]. The observed shift in the position of the $1415 \mathrm{~cm}^{-1}$ band towards a higher wave number as crystallinity decreases has been associated with a lower effectiveness of interchain interactions within the lattice, and hence with lateral disorder in the crystals [243]. These authors have also compared the experimental data with DFT-LDA based calculations detecting good qualitative agreement, taking into account that the models considered an infinite crystal and did not include Fermi resonance 
coupling. Moreover, in a recent study performed by Galimberti et al. [263] designed to describe the IR spectra of PE in the crystalline state as obtained through DFT calculations, it was found that intermolecular interactions occurring when chains are packed in the crystal are far from being negligible for a good prediction of solid crystalline phase spectra. Moreover, comparing spectroscopic quantities (i.e., IR and Raman intensities), predicted for an isolated unit and for a unit belonging to a crystal, requires some caution.

Recently, in a DFT study including dispersion corrections, the slip mechanisms and ground state twin configurations in crystalline orthorhombic PE were assessed to accurately characterize PE crystal defects and plasticity mechanisms [264]. Winey et al. [265] reported that the polymer chains are folded in different ways depending on the type of branching. Non polar functional groups are excluded from the crystal, which spans only the crystallisable ethylene sequence between the branches [266]. This should lead to thin crystals and switchboard-like amorphous regions. Polar functional groups may direct the chain folding in a hairpin manner near each side branch, giving rise to multiple embedded layers, in agreement with computer simulations.

\subsubsection{Crystallization of single chains}

Pioneer MD simulations were performed by Kavassalis et al. [267, 268] for single linear PE chain nucleation under vacuum using Dreiding II FF. These authors showed a clear transition in linear PE chains from a globular state to a chain-folded crystallite with a hexagonal symmetry, in which the driving force for crystallization was dominantly van der Waals attraction between constituent atoms. In subsequent studies [269-271] it was reported that: (i) although resultant interchain distances were of the order of those expected, lamellar thicknesses $\left(l_{c}=4 \mathrm{~nm}\right)$ were, however, quite lower than those derived from solution-growth single crystals $\left(l_{c}=10-12 \mathrm{~nm}\right)$ [244]; (ii) both non-adjacent and adjacent reentry were observed in a ratio of around 3:1; and (iii), two transitions were identified as coil-to-globule and globule-to-folded chain, dominated by Van der Waals and torsion energies, respectively [272, 273]. A series of MC simulations has been reported by Frenkel's groups concerning the free-energy barrier that separates the crystalline state of a homopolymer chain from the disordered state [274-276]. These observations are in close agreement with single chain experiments $[277,278]$

The effect of SCB on single PE chain crystallization in vacuo has been explored by Doran and Choi [279], Zhang et al. [280, 281] and Sharkh and Hussein [282]. In these studies, interesting phenomena were observed including a decrease in the trans/gauche ratio and crystal sizes with $\mathrm{SCB}$, branch inclusion in the crystals for systems containing the shorter branches, exclusion of longer branches from the crystals and inhibition of crystallization for systems with more than $50 \mathrm{SCB}$ per 1,000 C atoms. All these phenomena are in fairly good agreement with experimental observations in single crystals of SCB PE [244, 259]. 

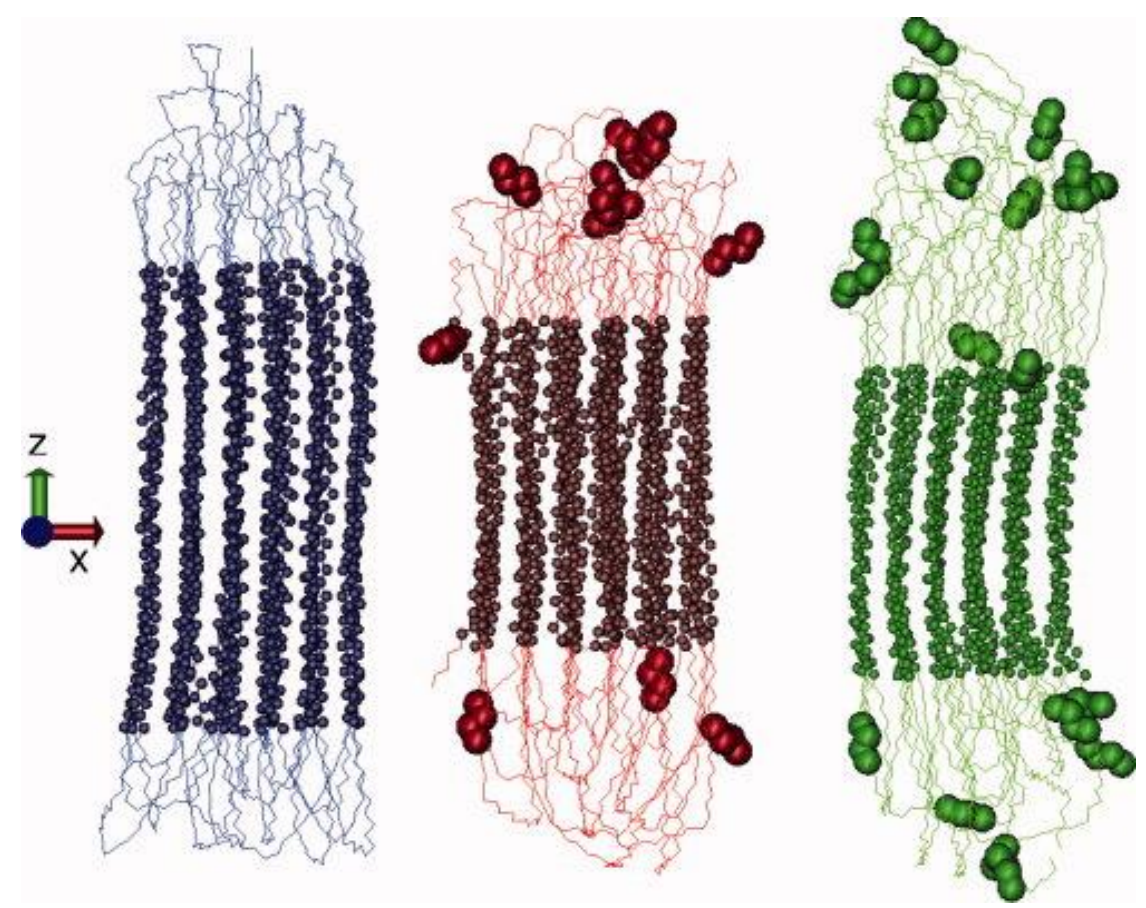

Figure 11. Selected geometries for PE-00 (blue), PE-05 (red), and PE-10 (green) models showing lamellar structures for a single-chain. Reprinted with permission from J. Ramos, J. Martinez-Salazar. J. Polym Phys., 49:2011,431-430. Copyright 2011 Wiley Periodicals, Inc.

However, a more realistic description of the systems that explicitly takes into account solvent molecules is not an easy computational task. A possible approximation is to consider only solvent viscosity using a Brownian-dynamics (BD) or Langevin dynamics (LD) method. Muthukumar et al. considered crystallization of a single chain by the LD-method [283, 284]. In their simulations, a nucleation and growth mechanism gives birth to some initial crystal nuclei (baby nuclei) in the early stages of homogeneous crystallization. Free energy barriers dictate initial lamellar thickness. Once the baby nucleus has formed, chains diffuse to the growth front where they are simultaneously adsorbed and crystallographically attached. This step is not hindered by a barrier, in contradiction with the underlying assumptions of the LH theory. The newly added folded chains undergo a rearrangement on the growth front to form stems that are commensurate with crystal thickness at the growth front. Meanwhile, chain dynamics within the crystal results in overall thickening of the lamella. This model has been widely used to examine certain aspects of single chain crystallization in solution such as the nature of the loops [285], the free-energy landscape [285-287], and the effect of SCB [288, 289]. Stems, branch positions, and folding surfaces are shown in Figure 11 for selected final structures of random ethylene/1-hexene $\mathrm{C}_{2000}$ chains with different contents of 1-hexene (from 0 to $10 \mathrm{SCB}$ every 1,000 $\mathrm{C}$ atoms).

As may be observed, the butyl branches are not incorporated within the crystalline region, rather they remain in the amorphous phase. On the contrary, it was found that methyl branches can be partially included in the crystals. On close inspection of the semicrystalline state through the local bond-orientational parameter, we can define the crystalline stem length and overall crystallinity of the models: both magnitudes decrease with SCB content, as in experiments [244, 259]. Recently, the effects of both different types and contents of precisely placed SCB and chain length (up to $\mathrm{C}_{10000}$ and polydisperse systems) have been explored [290, 291]. Results confirm that the branch acts as a defect both for nucleation and growth processes, decreasing the crystallization 
rate, crystallinity, and lamellar thickness. Moreover, both SCB and chain length have strong effects on the final morphology of the crystals. These authors showed also that branch length seems not to influence crystallization kinetics. They also found a critical SCB content for a morphological change from lamellar to bundle structures, at around $20 \mathrm{SCB}$ per 1,000 $\mathrm{C}$ atoms. Interestingly, they detected a trans-rich phenomenon in the pre-crystalline state and a trans-state critical population for crystallization onset that is independent of SCB content and length.

\subsubsection{Crystallization from the melt}

So far, reports exist of Monte Carlo as well as atomistic and CG MD simulations of the crystallization of flexible polymers, especially PE. Through MC simulations, $\mathrm{Hu}$ [292] and Xu and Mattice [293] identified interesting features such as integral folding of short chains in crystallites and a constant linear crystal growth rate, along with roughness of the local growth front. They also assumed that crowding of dangling ends on the fold surface was the main mechanism for suppressing the lateral growth front, proposing the mechanism of chain folding. These studies also showed that a high degree of crystallinity can be achieved in a reasonable computer time. In addition, the simulations revealed that segregation of low-molecular-weight fractions in the crystal growth of polydisperse polymers is a general phenomenon [294] and also the typical regime transitions associated with the Lauritzen-Hoffman model [295]. However, the predicted smooth crystal growth front in regime I was not shown by these simulations. MC methodologies have been also applied to different types of copolymer bearing crystallizable and non-crystallizable units [296]. In this case, the overall composition and statistical distribution of the monomers affect phase-transition, resultant relative crystallinity and crystal morphology, as indicated by the experiments. More recently, Gao et al. [297] reported a strong memory effect of crystallization in ethylene-based homogeneous random copolymers after their annealing at temperatures higher than the equilibrium melting point. This phenomenon has been associated with sequence-length segregation which leads to a heterogeneous melt in which the local concentration of long sequences raises the melting point.

For atomistic simulations, two different FFs (PYS-UA and TraPPE-UA) are being widely used to examine the crystallization process. Rutledge's group has extensively used PYS-FF, with minor modifications. These authors reported that their version captures essential qualitative aspects of the initial stages of the crystallization of nalkanes and short chains of linear PE up to $C_{100}$ [298-301]. In particular, these works have shown that homogeneous nucleation of PE models is feasible for deep supercooling using MD simulations. Further, they have characterized the crystal nucleus as a cylinder shape to calculate crystal thickness as a function of nucleus size. Their model predicts growth rate as a function of temperature and molecular weight up to the entanglement molecular weight. They also find evidence of a surface nucleus involving 4-5 chain segments approximately 20 beads long, shorter than the final thickness of the chain stem in the crystal, and involving segments from multiple chains. Remarkably, a crystal growth rate equation, parameterized from the MD [302], can be obtained, which works well for alkanes and PE using the Rouse relaxation time for n-alkanes and the local relaxation of an entangled segment to describe the kinetic barrier for crystallization. However, the model supports different growth mechanisms for fast and slow cooling. More recently these authors conducted MD simulations in longer PE systems (up to $\mathrm{C}_{1000}$ ) [233], that can calculate the nucleation rate and identify critical nuclei via a mean first-passage time analysis. The nucleation rate was found to be insensitive to chain length in the molecular range explored at deep supercooling. The 
critical nucleus was reported to contain about 150 carbons. Chain segment analysis gave rise to a broad length distribution of loops, which supports the "switchboard model" in the early stage of crystals formed in deep supercooling. Recently, refinements of the MD procedure and a kinetic model have been described to explain surface nucleation and crystal growth in $\mathrm{C}_{50}$ systems [303, 304].

Anwar et al. also applied MD methodologies using PYS-FF to explore homogeneous nucleation and growth processes in $\mathrm{PE}\left(\mathrm{C}_{20}-\mathrm{C}_{500}\right)$ [305-307]. For the nucleation process, they noted that the chains first align themselves and then straighten. As a consequence, local density increases and finally the monomer units become positionally ordered. The subsequent crystal growth process is characterized by a sliding-in motion of the chains. Chains preferably attach to the crystalline cluster with one end, and then move along the stems of already crystallized chains towards their final position. This process is cooperative, i.e., neighbouring chains tend to get attached in clusters rather than independently. Once chains are attached, the lamella thickens by sliding of the segments along the long axis of the chain from the amorphous regions into the crystalline regions. These authors do not observe the formation of any folded precursors and the entanglements do not seem to have an effect on the nucleation mechanism.

Gee et al. modified the parameters of the PYS-UA to enhance computational efficiency when examining the crystallization process in polymers [308, 309]. However, the torsion potential was substantially modified increasing the trans to gauche energy barrier as compared to the bond torsion potential of the original FF. In this way, chain stiffness increases, enhancing computational efficiency through acceleration of the process. This procedure enabled these authors to observe forced crystallization at time scales accessible via large-scale MD simulations. In the ordered domains, they identified dense growing regions similar to smectic liquid crystals. The MD simulations revealed a 'coexistence period' in ordering before crystallization, during which nucleation and growth mechanisms coexist with a phase-separation mechanism.

Yamamoto et al. used a specific FF to address different aspects of the crystallization of PE chains (up to $\mathrm{C}_{1000}$ ) from both quiescent and oriented melts in the bulk and/or between surfaces [310-313]. They adopted a simplified molecular model for polymethylene-like chains. The chain comprises $\mathrm{CH}_{2}$-like beads connected by harmonic springs, and the lowest energy conformation is a linear stretched sequence of the beads with slight bending stiffness imposed. Although they found no precursor prior to crystallization, they did nevertheless observe the growth of stacked chain-folded lamellae from the substrates. The growing lamellae showed thickening growth along the chain axis as well as usual growth perpendicular to it. They reported the characteristic bell shape dependence of crystallization rate with temperature, and a maximum around $325 \mathrm{~K}$. In addition, the fold surfaces were found to be covered by relatively short chainfolds; at least about $60-70 \%$ of the folds connected the nearest or next nearest neighbour crystalline stems. In subsequent modifications of the model, they were able to simulate larger systems [314-317]. Detailed statistics of folds and cilia revealed that the folds are rather neat and mostly make re-entries into the nearest stem positions (adjacent-reentry), whereas the cilia are generally short but with a small number of longer cilia forming thick amorphous layers. Conformational analysis of the chains forming nuclei revealed that the homogeneous nuclei are fringed micelle-like chain aggregates, but the chains as a whole have folded conformations.

The TraPPE-UA FF has been also recently used by Ramos et al. [73] to simulate the homogeneous crystallization process of linear entangled PE $\left(\mathrm{C}_{192}\right)$ from the melt at different cooling rates from $\Gamma=0.05$ to $1 \mathrm{~K} \cdot \mathrm{ns}^{-1}$. These authors described the 
characteristic drop produced in specific volume at the crystallization temperature, $\mathrm{T}_{\mathrm{c}}$. In addition, a continuous shift of the $\mathrm{T}_{\mathrm{c}}$ peak toward lower temperatures and a broadening of the transition with increasing $\Gamma$ are clearly observed. The value of $T_{c} \sim 325 \mathrm{~K}$ obtained with TraPPE-UA FF is still lower than those experimentally obtained by ultrafast calorimetry $\left(\mathrm{T}_{\mathrm{c}} \sim 370 \mathrm{~K}\right)$ for long n-alkanes $\left(\mathrm{C}_{122}, \mathrm{C}_{162}, \mathrm{C}_{390}\right)$ at $\Gamma$ of $10^{3}-10^{4}$ $\mathrm{K} \cdot \mathrm{s}^{-1}$. Likely, this is due to the fact that experimental $\Gamma$ values are 4 orders of magnitude lower than those accessible in the simulations. Interestingly, qualitative similarities can be observed between our simulations and recent crystallization experiments for linear PE performed using ultrafast calorimetry by Toda et al. [318]. Both the experimental shift to lower temperatures and transition broadening as the cooling rate increases are adequately described by both simulations. Also a considerable decrease in the degree of crystallinity is observed as the cooling rate increases. At the end of the MD simulations, the simulated chains were mostly once- $(36 \%)$ and twice(38\%) folded. Fascinatingly, a large percentage of these once- and twice-folded chains closely fold into a "quasi" integer shape (around 20\% of the total number of chains). Also, but to a lesser extent, we can observe thrice-folded (10\%) chains and chains with tie segments $(16 \%)$ with their ends passing through more than one ordered domain. Further, in the ordered domains, the adjacent folded-structure is not the only one present. The picture supports the "switchboard" re-entry model, at least for early crystallization stages at high supercooling temperatures. At these early crystallization stages, clear segregation takes place, as a process similar to that observed during crystallization from a pre-oriented melt by Ko et al. [319] and Yamamoto [320]. At the end of the simulation process, it is possible to observe different ordered and disordered layers. In the amorphous layer, the tie, cilia and loop segments of the chains are concentrated, resembling the model proposed by Ungar and co-workers for quenched long-alkanes of similar molecular length $\left(\mathrm{C}_{198}\right.$ and $\left.\mathrm{C}_{210}\right)$ [2, 321-324]. In this model, a mixed-integer folded-extended phase forms instead of the pure once-folded phase typical of high n-alkanes. Rather than re-entering the existing crystal layer, cilia from neighbouring non-integer folded (NIF) lamellae gather and form an intercalated layer.

The MD simulations have also enabled assessment of primary fold stem length, $1_{\mathrm{g}}{ }^{*}$. The most common definition of stem length is that given by Lacevic and co-workers [309]. It has been long recognized that $1_{\mathrm{g}}{ }^{*}$ in melt-crystallized PE is exclusively dependent on the extent of supercooling [325-330]. Results obtained from atomistic MD simulations of the melt are in good quantitative agreement with experiments, as may be observed in Figure 12.

The classic Gibbs-Thomson-Hoffman theory has led to a well-known expression for the critical thickness $1_{\mathrm{g}}{ }^{*}=\mathrm{K} / \Delta \mathrm{T}+\delta$, where $\mathrm{K}=2 \sigma_{\mathrm{e}} \mathrm{T}_{\mathrm{m}}{ }^{0} / \Delta \mathrm{H}_{\mathrm{f}}{ }^{0}$ (where $\sigma_{\mathrm{e}}$ is the fold surface energy, $\mathrm{T}_{\mathrm{m}}{ }^{0}$ is the equilibrium melting temperature and $\Delta \mathrm{H}_{\mathrm{f}}{ }^{0}$ is the heat of fusion at $\mathrm{T}_{\mathrm{m}}{ }^{0}$ ) and $\Delta \mathrm{T}=\mathrm{T}_{\mathrm{m}}{ }^{0}-\mathrm{T}_{\mathrm{c}}$ is the level of supercooling. The parameter $\delta$ is empirical, and it was introduced to account for the non-zero value of $1_{\mathrm{g}} *$ at large supercooling upon linear extrapolation of experiments to $[1 / \Delta \mathrm{T}]=0[331]$. The linear correlation explains well the experimental results for $T_{c}$ values from $401 \mathrm{~K}$ to $360 \mathrm{~K}$ (Figure 12). A value for $1_{\mathrm{g}}{ }^{*}$ of $6 \mathrm{~nm}$ has been recently obtained in single lamella PE nanoparticles synthesized at $\mathrm{T}=288 \mathrm{~K}$ [332]. This value deviates from the linear correlation (open circle in Figure 12), and the authors argued that this is not exactly the initial fold length at that temperature, as some degree of thickening is expected at the temperature at which the thickness was measured. At this large level of supercooling we find the advantage of MD simulations. Recent simulations have reported values for $1_{\mathrm{g}} *$ decreasing from $6 \mathrm{~nm}$ to $3 \mathrm{~nm}$ for $\mathrm{T}_{\mathrm{c}}$ values of $370 \mathrm{~K}$ down to $280 \mathrm{~K}$ [64, 306, 320, 321] (solid symbols in Figure 12). The trend in Figure 12a shows a levelling off $1_{\mathrm{g}}{ }^{*}$ as $\mathrm{T}_{\mathrm{c}}$ 
decreases towards $\mathrm{T}_{\mathrm{g}}$ (around $187 \mathrm{~K}$ in our simulations), similar to that anticipated by the experimental linear extrapolation. However, a slight deviation is shown by these recent simulations at lower temperatures. It would be desirable to explore a larger degree of supercooling in order to determine the trend in the vicinity of the glass transition. However, these MD simulations represent a challenge as crystallization kinetics is very slow in the vicinity of the glass transition.
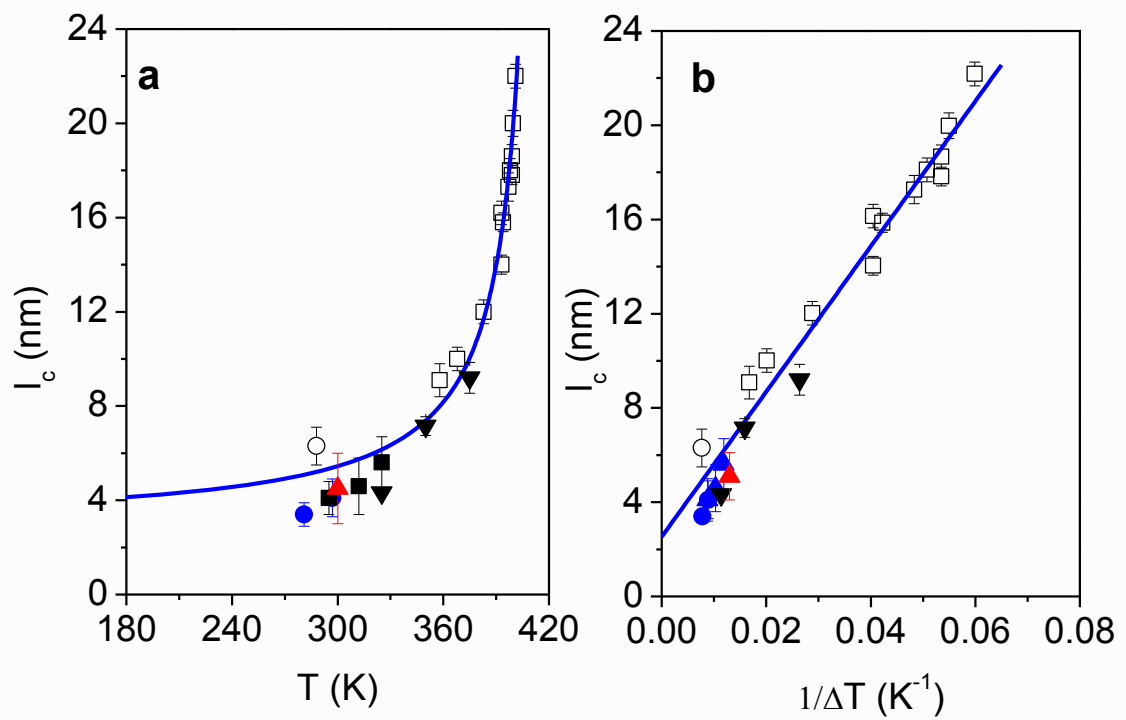

Figure 12: Initial fold length against (a) crystallization temperature and (b) the reciprocal level of supercooling, obtained for simulated crystalline structures: C192 (O) PYS-UA and ( $\mathbf{\square})$ TraPPE-UA [73]; single PE chains in solution $(\boldsymbol{\Delta})$ simulations [333, 334]; $(\boldsymbol{\nabla})$ quenched preoriented melts [319] and experiments: ( $\square$ ) PE [325-327] (O) single PE lamella [332]. The solid line is the best fit of the experimental results. Reprinted with permission from J. Ramos, J.F. Vega, J. Martinez-Salazar. Macromolecules, 48:2015, 5016-5027. Copyright 2015 American Chemical Society.

As mentioned in the preceding section, the effect of SCB in the crystallization process has been also explored through MD simulations. Notwithstanding, most results correspond to single chain systems. Further, according to most results, the branches act as nucleation seeds, which are later rejected during crystal growth. The number, length and flexibility of the branches play an important role in determining the formation of the lamellar structure. The longer the branch length and fewer the branch sites, the more perfect the formed lamellar structure will be. In all these works, the characteristic general trends observed experimentally in SCB polyolefins are noted, such as rejection to the amorphous regions of branches longer than methyl, and a decrease in crystal size and melting temperatures with SCB content [121-123, 243, 244, 335-337].Very few simulations can be found to study the effects of SCB on polymer crystallization from the melt. Choi et al. examined solid-state structures formed in the early stage of crystallization of different homogeneous and heterogeneous LLDPE models by means of MD simulations using the DREIDING FF [338, 339]. These simulation results show that for homogenously branched systems, SCBs are distributed in both the interphase and the amorphous regions. However, the SCBs are more likely to be located in the amorphous phase for heterogeneous systems. Simulation results also indicate that, compared with homogeneous systems, heterogeneous ones have a higher degree of crystallinity and a longer average crystalline stem length. 
a)
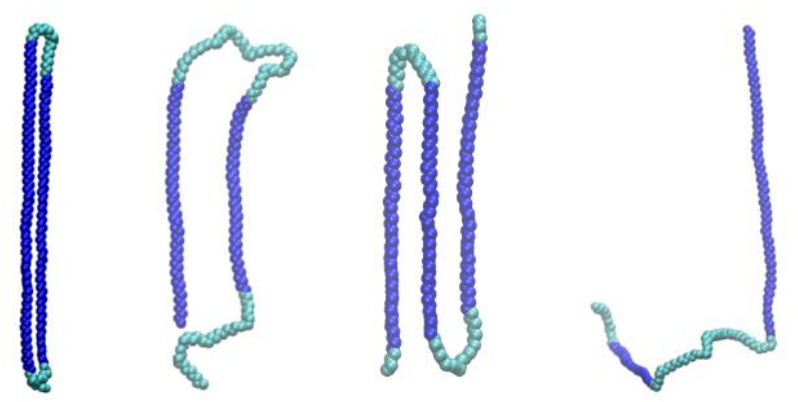

b)
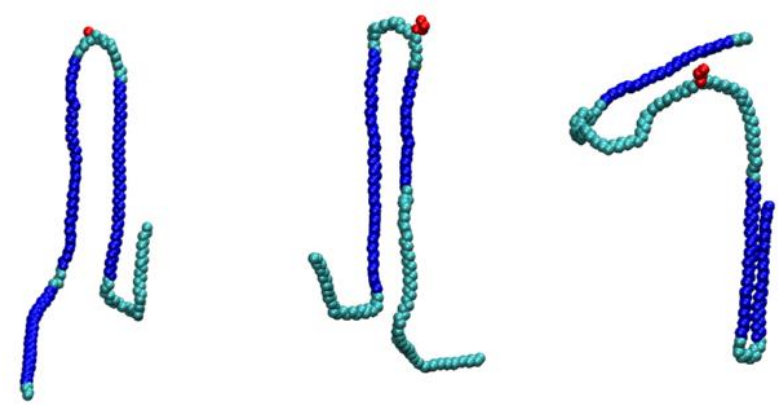

Figure 13. Chain conformational features at the end of simulation runs from the melt for: (a) linear and (b) butyl branched C192. The ordered stems and branch are shown in blue and red, respectively. The chains were extracted from the whole system and segments from other chains are not shown for clarity. Reprinted with permission from S. Sanmartín, J. Ramos, J.F. Vega, J. Martinez-Salazar. European Polymer Journal (2014) 50, 190-199. Copyright 2013 Elsevier Ltd.

Sanmartín et al. recently undertook MD simulations on a series of "precisely" branched ultralong $n$-alkanes to shed light on the early stage processes of crystallization from the melt at high undercooling temperatures using TraPPE-UA FF [340]. This study considers a linear $\left(\mathrm{C}_{191} \mathrm{H}_{384}\right)$ and two symmetrically branched $\left[\mathrm{C}_{96} \mathrm{H}_{193} \mathrm{CH}\left(\mathrm{CH}_{3}\right) \mathrm{C}_{94} \mathrm{H}_{189}\right.$ and $\left.\mathrm{C}_{96} \mathrm{H}_{193} \mathrm{CH}\left(\mathrm{C}_{4} \mathrm{H}_{9}\right) \mathrm{C}_{94} \mathrm{H}_{189}\right]$ ultralong n-alkane samples. First of all, these authors observed that SCB provokes a delay in crystallization as compared to the linear chain. The process is further delayed as branch length increases. The time evolution of the structure factor $S(q)$ as the materials are undercooled, revealed that both crystal thickness and chain packing were dramatically affected by the presence of SCB. Crystallinity also decreases as SCB length increases. The folding observed in the linear system was closer to the irregular model of folding at least for the early stages of crystal nucleation and formation. The length of the branch clearly determines its inclusion or not in the nuclei, and consequently disturbs the formation of crystals in a different way. The chain conformational analysis indicates that most of the chains in the linear model adopt once-fold configurations from the initial stages of the nucleation process, but tie, and twice-fold chains are also already present. Most interestingly, the number of carbon atoms involved in the most perfect folds are some $8 \pm 2$, which entirely matches the results reported experimentally by Grasso and Titman [341]. In the branched samples, the once-fold morphology seems predominant, obviously because of the presence of the branch in the middle of the chains, which favours once-fold chain structures from the process onset (see Figure 13).

Certain aspects of the impacts of molecular architecture on the crystallization and the subsequent annealing process remain practically unexplored. We have extensively studied this phenomenon and its effect on macroscopic properties in the past $[342,343]$ yet computer simulations are still a challenge, as both chain dynamics and morphology in these events cover a wide range of time and length scales that are outside the scope of conventional atomistic simulations. 


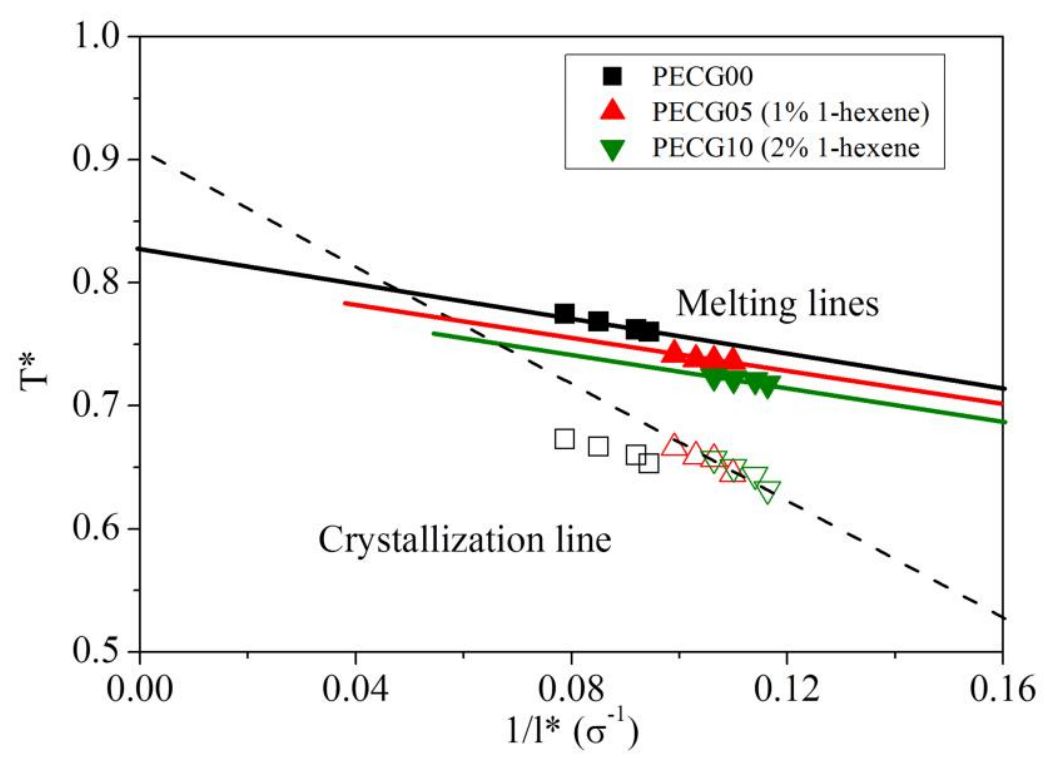

Figure 14: Simulated crystallization (at different cooling rates) and melting (at a heating rate of $\Gamma=5.0 \times 10^{-6} \tau^{-1}$ ) lines for three CG models of PE: linear, $1 \% 1$-hexene and $2 \% 1$-hexene. Reprinted with permission from J. Ramos, J.F. Vega, S. Sanmartín, J. Martinez-Salazar. European Polymer Journal (2016) 85, 478-488. Copyright 2016 Elsevier Ltd.

To analyze such large scales, CG simulations are an interesting option, as they allow us to increase not only the size of the systems under study, but also the observation time window. Basic studies have reported interesting results, mainly with regard to the crystallization process in CG models of polyvinyl alcohol (PVA) [344, 345]. In these studies, the characteristic chain folding and lamellar structure along with the typical features of a first order transition are observed. Remarkably, the relationship between crystallization and melting temperatures with the inverse of lamellar thickness is reproduced [346]. In addition, typical phenomena of flexible polymers have been reproduced, such as the coexistence of melting and growth during heating and crystal thickening during isothermal crystallization [347-350]. More recently, interesting MD simulations have been performed using CG-PVA [351-353] and CG-PE models (see supporting information in [354]) connecting the crystallization process with the entangled polymer state. Topological analysis reveals the existence of disentanglement processes during crystallization that compete with the tightening of entanglements during cooling caused by local stiffening of chains. In addition, a direct relationship emerges between the temperature dependence of entanglement properties and crystal thickness. These results highlight the importance of the study of entanglement properties at different temperatures in crystallisable polymers of different molecular architectures to establish the complex relationship between entanglement state, crystallization and final lamellar structure. 


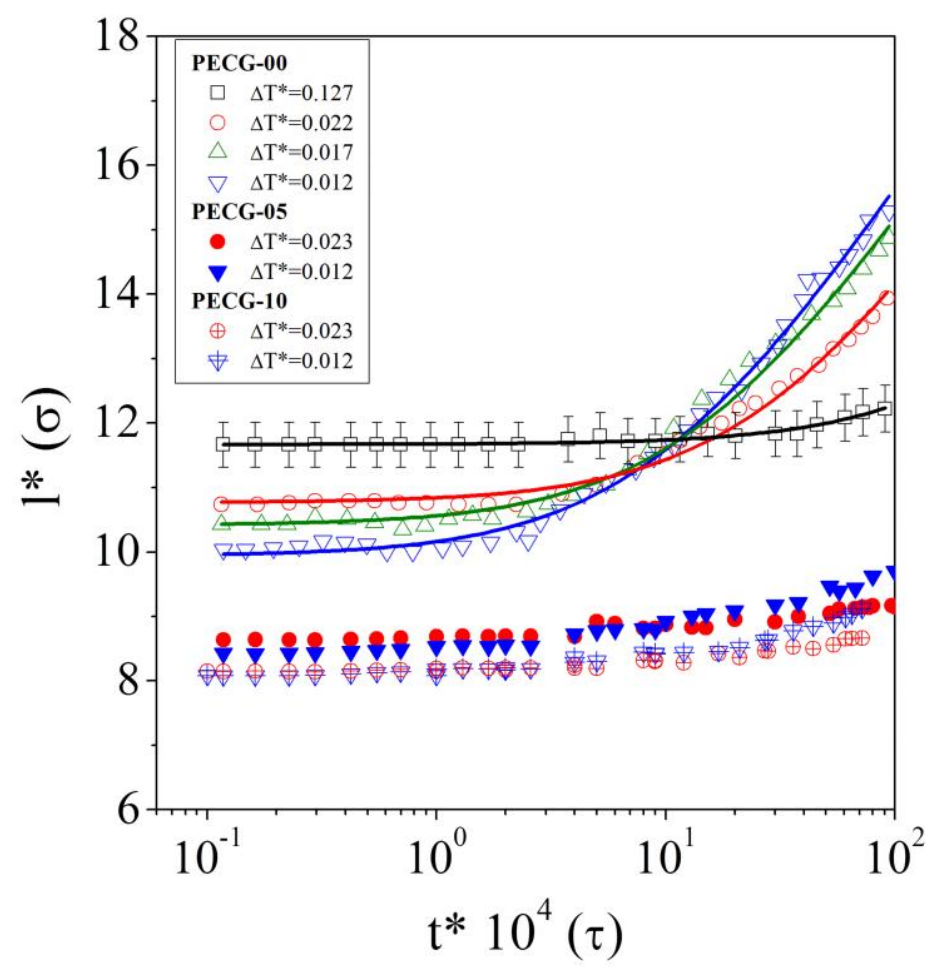

Figure 15: Stem length time evolution according to the degree of undercooling for three CG models of PE systems. The same colour means the same undercooling. Reprinted with permission from J. Ramos, J.F. Vega, S. Sanmartín, J. Martinez-Salazar. European Polymer Journal (2016) 85, 478-488. Copyright 2016 Elsevier Ltd.

Vettorel et al. [355] and Ramos et al. [356] have developed and optimized CG models for linear and branched PE. These authors used these models to examine the crystallization of short linear chains (mainly, n-alkanes) and branched PEs of up to 10 SCB per 1,000 C atoms. It has been shown that crystallization and subsequent melting temperatures are related linearly to the inverse of lamellar thickness. Different melting lines are obtained according to the SCB content, and a trend is observed toward a single crystallization line. These data qualitatively match the experimental lines obtained in model homogeneous ethylene/ $\alpha$-olefin copolymers [357], as illustrated in Figure 14.

Additionally, a strong lamellar thickening process is reported for the linear model at temperatures below the melting point, which follows the classic interpretation reported in PE, whereby a logarithmic increase in crystal thickness occurs with time (see Figure 15) [358-362]. However, for branched samples, thickening is impaired at the same degree of undercooling. In branched models, SCB are excluded from the crystal and remain on the surface and in amorphous regions. This causes the pinning of these regions, minimizing crystal chain diffusion and hindering crystal stem thickening, in qualitative agreement with the experimental data [357]. It should be noted that the recent formulation of Salerno et al. for "coarser" CG PE models might also be adequate to address the crystallization of linear and SCB PE systems [94, 95]. These authors have already reported a pronounced density increase for CG levels of $\lambda \leq 4$ (see Section 4.3.1) and cooling rates of $1.4 \mathrm{~K} / \mathrm{ns}$ related to crystallization processes around temperatures of $250-330 \mathrm{~K}$ for the $\mathrm{C}_{96}$ chains. These transitions are observed in the same range of temperatures as those detected in atomistic MD simulations of a $\mathrm{C}_{192}$ longer system for cooling rates within the range $1-0.05 \mathrm{~K} / \mathrm{ns}$ [73]. Thus, these CG models represent an advanced simulation methodology to allow for longer time-length scales to examine the crystallization process in PEs with different topologies 


\subsubsection{Nature of the amorphous region in lamellar polyethylene}

Semicrystalline PE typically consists of amorphous layers sandwiched between crystalline lamellae. This morphology is present in both linear and SCB PE, at least up to comonomer contents of around 6 mole $-\%$, or crystallinity around 30\% [363]. The non-crystalline region, also known as the interlamellar phase, has no clear structure, but there is general agreement it can be viewed as a central amorphous core between rigid amorphous layers (interphase) near to the crystals (see Figure 16) [364].

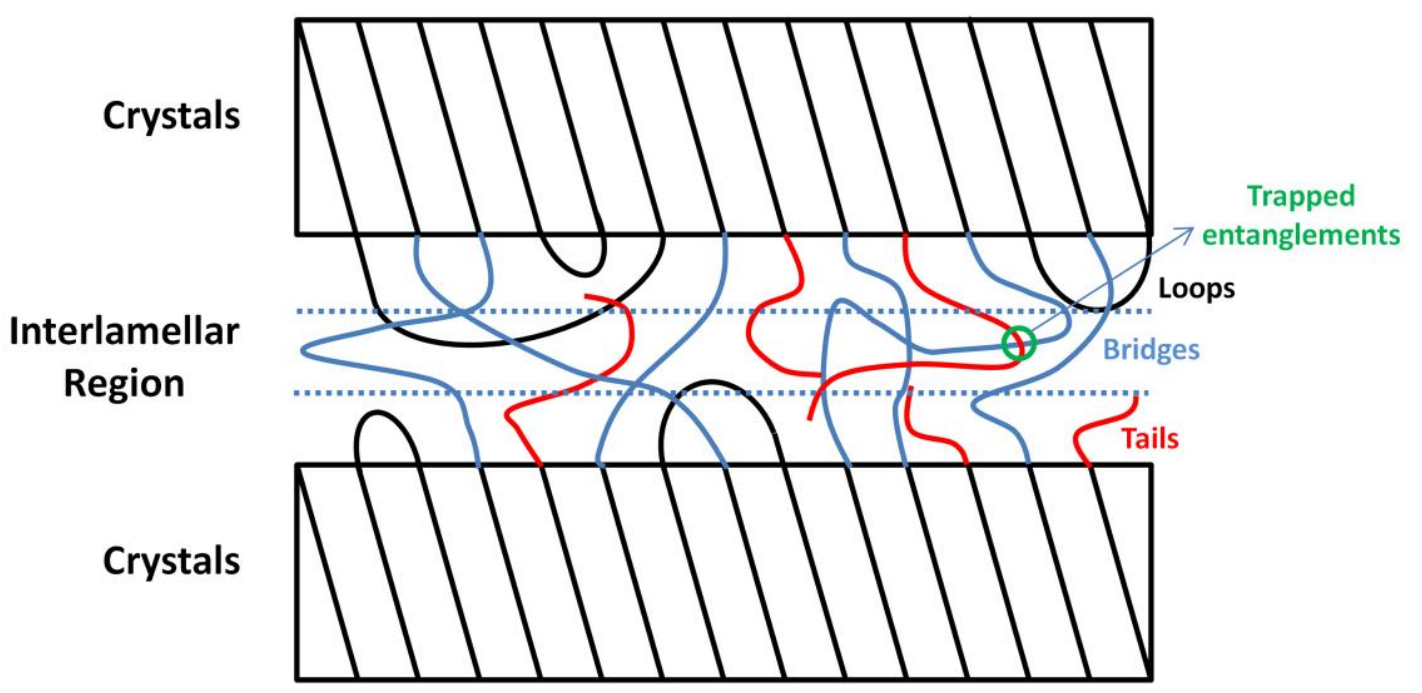

Figure 16. Diagram illustrating the semi-crystalline structure of PE with examples of tails, bridges, loops and trapped entanglements within the interlamellar region.

The rigid amorphous region is not well known, but there has been long interest in identifying its conformational and mechanical properties. The topological characteristics of the chain segments within this region are the following: (i) chain segments that connect different crystals in the interlamellar region (tie chains or "bridges"), (ii) segments that enter back into the same crystal ("loops"), and (iii) segments that end in the interlamellar region ("tails"). These topological features together with the characteristic layer stacking are the foundations of the molecular picture of the semicrystalline state, which is well established in linear model PE samples [365].

Notwithstanding, a detailed study of the complex relationships between chain molecular and topological aspects, structure, morphology and properties has always been a challenge for this implicit picture. A first level of description of the mechanical properties of semicrystalline PE is to consider three independent but coupled phases. Sedighiamiri et al. applied micromechanical modelling to semicrystalline PE by considering an interphase with intermediate properties between amorphous and crystalline regions [226]. Martín et al. also modelled PE as a three component system in which interface properties are monitored by its rigid amorphous fraction content, dependent on molecular structure, as determined through Raman experiments [366]. This kind of micromechanical modelling is useful to empirically reproduce the properties of the interphase, and to extract information about the effect of molecular architecture on the amorphous regions.

To gain further insight into the molecular features of this region, MC and MD simulations are being intensively applied to the case of PE. Hütter and 't Veld et al. 
undertook the first theoretical estimates of the thermal and mechanical properties of the "interlamellar phase" of semicrystalline linear PE by MC simulations using the PYSUA FF or modified versions [367, 368]. Using this interface MC (IMC) model and micromechanical homogenization approaches [369], it is possible to dissociate interfacial stiffness from that of the whole interlamellar region. The interphase thickness and conformational aspects of the chain segments in this region, together with the phase volumes, can be also deduced. These simulations have shown that this phase exhibits properties that are intermediate between those of the crystal phase and the amorphous melt, but verification is still not possible, as no direct access exists to experimentally measure these properties. The crystalline region and its related properties during deformation are quite well understood as direct access to structural features is possible [370]. However, straight characterization of the amorphous regions in terms of loops, bridges, tie chains, and more importantly, trapped entanglements is much more difficult. Through IMC plus MD, both the conformational aspects of the interlamellar region can be examined and the time evolution of this component can be followed in conditions of tension [371, 372], compression and shear [373]. Today, the complexity of systems increases, as the effect of layer thickness and chain tilt in crystalline regions is under examination [374]. It these works, it has been shown that the initial interlamellar phase in semi-crystalline PE is more entangled than the amorphous PE melt. It is speculated that the origin of this greater entanglement density is the confinement of the amorphous component between crystalline lamellae. During the deformation process, the density of entanglements is reported to distinctly vary with the content of non-crystalline material, the mode and the rate of deformation. In general, it has been shown that there is competition between the thermodynamic stability of the crystals and displacement of the chain segments in the different regions. At high strain rates, the stress-strain curve shows the typical yield accompanied by cavitation in the amorphous region, but at lower strain rates, cavitation is suppressed and strain hardening emerges, as in experiments. Simulations suggest that higher levels of topological constraints in the interphase such as short "bridge" segments can be correlated with this strain hardening. Hence, changes in the interphase could lead to differences in strain hardening behaviour after the yield. This is quite interesting as the nature of the interface region can be modulated by the molecular architecture of the polymer. As molecular weight and SCB content affects both crystallinity and the nature of the amorphous regions, not only the elastic modulus and microhardness [375-377] but also yield behaviour and strain hardening will strongly vary with SCB [258, 363, 366, 378, 379]. An example is presented in Figure 17, in which experimental nominal tensile stress is plotted against the nominal tensile strain, in percent, for a series of rapidly quenched SCB PE samples with increasing SCB contents yet the same molecular weight $\left(\sim \mathrm{M}_{\mathrm{w}}=130 \mathrm{~kg} / \mathrm{mol}\right)$ and molecular weight distributions $\left(\mathrm{M}_{\mathrm{w}} / \mathrm{M}_{\mathrm{n}} \sim 2\right)$ (model single-site ethylene/1-hexene copolymers) [366]. All the samples in Figure 17 display ductile behaviour, the yield being well-defined. Thus, the yield becomes more diffuse as SCB increases and the value of the yield stress decreases. The same trend is found in linear PE samples as molecular weight increases [378]. This behaviour is a replication of decreasing crystallinity level with SCB content. Subsequent to the yield point, stress falls, after which there is a region samples where stress changes little with the applied strain. This region is followed by strain hardening behaviour. With increasing SCB, the plateau region beyond yield decreases, and the slope of the strain hardening region becomes steeper, with a levelling off beyond 8 branches every 1,000 $\mathrm{C}$ atoms. This general behaviour is observed in high molecular weight SCB PE [379]. 


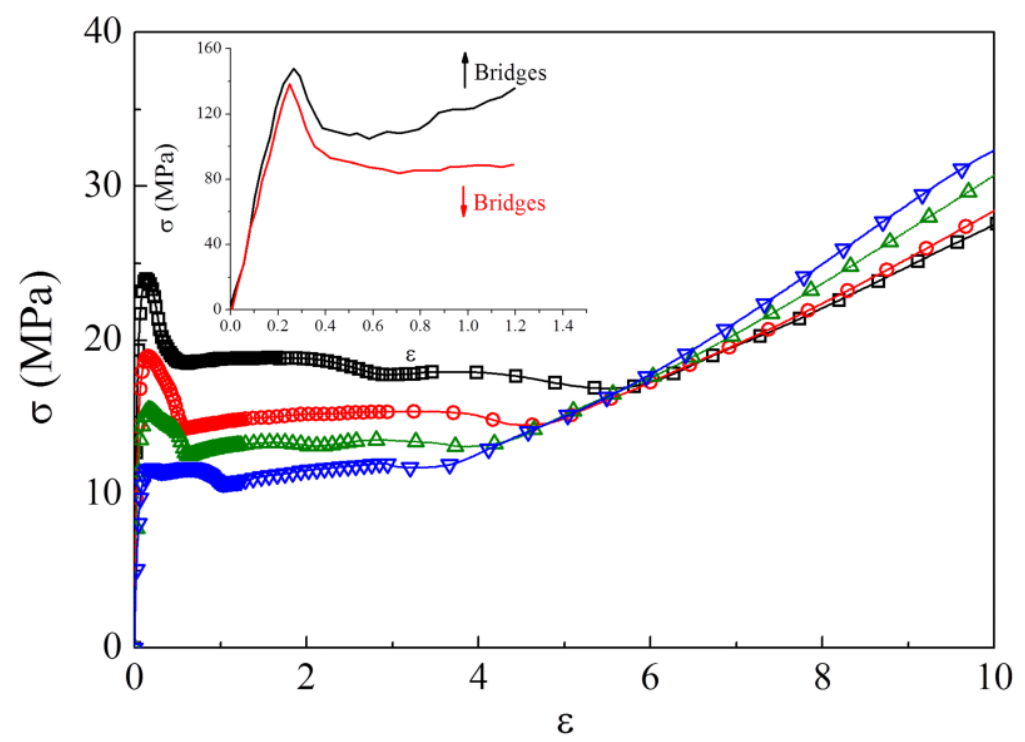

Figure 17. Tensile stress-strain curves for representative SCB PE samples (ethylene/1-hexene copolymers) of different SCB contents at room temperature: ( $\square)$ PEH0, (O) PEH3, $(\triangle)$ PEH5, ( $\nabla)$ PEH8 and $(\diamond)$ PEH10. Stress and strain are based on undeformed sample dimensions. The results shown in the figure are extended data to those presented in reference [366]. The inset shows the simulations performed by Yeh et al. [374] for semicrystalline PE containing different concentration of tie chains.

Recent computer simulations performed on linear PE by Yeh et al. [374] suggest that higher levels of topological constraints in the interphase lead to enhanced strain hardening behaviour (see inset to Figure 17). The question that arises is whether the rejected SCB in the amorphous phase could give rise to more constraining in this region. There are very few studies in the literature describing computer simulations for the specific case of SCB PE samples, but interesting works have been recently reported by the groups of Gedde [380, 381] and Rutledge [382]. Gedde's group has developed an MC code to assess tie chain and trapped entanglements concentrations in the interlamellar region in linear and SCB PE. This study shows an increase in both tie chains (bridges) and trapped entanglements, and therefore of interlamellar connections, as SCB and molecular weight increase. This is undoubtedly related to the greater strain hardening behaviour observed experimentally in SCB PE samples (Figure 17) and improved crack growth resistance reported in the past [383]. In this regard, the more recent IMC simulations using PYS-UA FF by Rutledge's group have generated opposing results. Thus, these authors found that SCB induce conformational behaviour and a preference for gauche states of the backbone segments. This directly affects the distribution of loops and bridges, which emerged to be less likely in SCB PE than linear PE. As indicated by Kumar et al. [382], more research is needed in this direction as the specific microstructure (size of the crystals and thickness of the amorphous phase) of the systems has an effect on the conformational populations of chains in the interlamellar regions.

\section{Brief description of the computational methodologies used}

Molecular modelling consists of a set of theoretical and computational techniques used to mimic the behaviour of molecules and materials. Computational tools can be understood as a series of algorithms implemented in a computer to numerically solve a model. Currently, computer modelling plays a prominent role in understanding the 
relations existing between the atomic structure and the macroscopic properties of polymers [33-38].

In the following sections, we provide a brief description of the main computational methods, their applicability to PE-based polymers and their limitations. Owing to space restrictions, rather than reviewing all simulation studies of PE properties, we here focus only on those that will help understand the topics covered in the previous sections. There are plenty of simulation studies using phenomenological models in which only some key aspects are captured to describe desired macroscopic behaviour. However, these do not have a clear or explicit connection to polymer chemistry. These models rather describe the universal behaviours of polymers. Thus, simulations based on general polymer models, albeit significant in polymer physics, are not covered here. Interested readers are referred to excellent reviews of simulation methods for polymers [30, 31, 384-387]. As a clear example of this kind of model, we should mention the bead-spring model proposed by Kremer and Grest [149], which has been widely used for investigating, among other properties, the complex dynamics of polymer systems due to the topological constraints (entanglements).

\subsection{Quantum scale. Ab initio quantum (QM) and density functional theory (DFT) methods}

Electrons and nuclei represent the degrees of freedom at the quantum scale. The characteristic length and time scales used in QM methods are around $10^{-10} \mathrm{~m}(1 \AA)$ and $10^{-12} \mathrm{~s}(1 \mathrm{ps})$, respectively.

The atomistic scale problem by means of traditional QM methods needs to solve the Schrödinger wave-function equation for $\mathrm{n}$ electrons and $\mathrm{N}$ nuclei within the BornOppenheimer strategy (wave-function formalism). The simplest approximation is the Hartree-Fock (HF) method, where it is assumed that each electron in a molecule is interacting with the average field created by the other electrons and the nuclei. However, the important correlation effects are not taken into account in the HF method, and to solve this problem more elaborate methodologies are needed either based on the perturbation theory (MP2, MP4, ...), coupled cluster (CC) or configuration interaction (CI) approaches. The interested reader is referred to the specialised books for more detailed descriptions [388]. In any case, the computational cost of these traditional QM approaches makes impractical its application to systems containing more than 20-30 atoms.

Another way to deal with the many-body problem is density functional theory (DFT). In DFT methods, we avoid calculating the n-electron wave function by using the electron density (density formalism) and Hohenberg-Kohn (HK) theorem within the Kohn-Sham procedure [389, 390]. The HK theorem shows that the exact ground state energy functional exists, depending only on electron density. However, the precise form of the potential is still unknown, and the construction of the exchange-correlation functional, $\mathrm{E}_{\mathrm{XC}}$, is the most challenging requirement. Thus, the selection of $\mathrm{E}_{\mathrm{XC}}$ is the most important issue when using DFT methods. There are a vast number of $\mathrm{E}_{\mathrm{XC}}$ functionals, among others, the most popular are the so-called local density approximation (LDA), generalized gradient approximation (GGA), meta-GGA and hybrid functionals [391]. The accuracy of calculation is mostly determined by the choice of this $\mathrm{E}_{\mathrm{XC}}$ functional.

However, traditional $\mathrm{E}_{\mathrm{XC}}$ functionals are unable to capture long-range weak dispersion (vdW) interactions. As a result, a range of extensions has been formulated to include such weak dispersive interactions in the DFT methods. Liu et al. have recently 
shown the importance of dispersion corrections for the accurate determination of structural properties of several polymers, among them PE [249].

Further, a basis set to describe the shape of the orbitals in an atom is required. The most used functions are, among others, linear combination of Slater-type orbitals, Gaussiantype orbitals or plane waves. Basis-set convergence is crucial for the accuracy of the calculations. The size and quality of the basis sets are identified using abbreviations, though these are too numerous to be described here [392].

Both QM and DFT methods are suitable to study processes involving the breaking/creation of bonds, changes in electron configurations and transfer charge phenomena. However, DFT methods have the advantage of a favourable computational cost/accuracy ratio compared with traditional correlated wave function QM methods [393]. Thus, larger molecular systems can be treated with adequate accuracy using DFT methods. Currently, systems containing about one hundred atoms can be simulated with DFT standard techniques. As a result, DFT is currently by far the most used quantum methodology

In summary, these methods are considered among the most accurate computational methods but are still computationally too expensive to apply to an ensemble of large polymer chains. In addition, many commercial and open-source computational codes are available for QM and DFT calculations [394, 395]. Some of the applications of firstprinciple methods, mainly DFT, to PE models are conformational and mechanical properties of single chain models [240, 245, 246, 248, 396-408], equilibrium unit cell, [239, 245-249, 396, 409], electronic properties [238, 401, 402, 407, 409-417], infrared and/or Raman spectra of the crystal state [243, 246, 263, 415, 418], inelastic neutron scattering spectra [245, 419], and Shock Hugoniot studies of PE crystals (studies of the material's behaviour under extreme conditions) [420, 421].

\subsection{Atomistic models based on classic mechanics}

Atomistic models are represented by the position of either all atoms (explicit or allatom models, AA) or interaction sites, grouping the $\mathrm{C}$ atom and its bonded $\mathrm{H}$ atoms (united atom models, UA) linked by a series of bonds, bend angles, and dihedral (proper or improper) angles. In these models, electrons are not explicitly included in the calculation, thus the energy of a system is calculated as a function of nuclear positions and their velocities. In this case, interactions between the sites are modelled using an empirical molecular potential, the so-called force field, which is a function only of the atom positions. The FF is generally divided in bonded (intramolecular) and non-bonded (intermolecular and intramolecular) parts. An example of FF is given in Equation (16). Terms in this expression are parameterized for each type of atom, bond, angle and torsion in the polymer chain. The types represent atoms, bonds, valance angles or torsion angles that are similar enough in the physical and chemical sense to be treated as identical in different molecular environments (e.g. an $\mathrm{O}$ atom in a carbonyl group, $\mathrm{C}=\mathrm{O}$ bond in a carboxylate group and so on). In other words, the principle of transferability of the same chemical groups is assumed [422]. The parameters can be obtained either by fitting experimental data or by high-level QM calculations of model systems containing the atom types. The accuracy of these models is highly dependent on the method used to parameterize the FF to describe a given physical phenomenon. Thus, validation of the model is of vital importance before using it to explain or make predictions of a physical phenomenon.

Simulations based on atomistic models are much faster that quantum mechanics and their computational requirements do not grow as fast with the number of atoms in 
the simulation. Thus, these models typically involve a number of atoms in the range of thousands to a few millions $\left(10^{3}-10^{6}\right.$ atoms $)$ packed in a simulation box spanning hundred of nanometres using periodic boundary conditions (PBC) to avoid spurious surface effects [78]. Time lengths are in the range of ps to $\mu \mathrm{s}\left(10^{-12}-10^{-6} \mathrm{~s}\right)$ for the largest simulations.

$$
\left.\begin{array}{rl}
V(\overrightarrow{\boldsymbol{r}})= & \sum_{i=1}^{\text {nbonds }} k_{i, \text { bondtype }}\left(l_{i}-l_{0, \text { bondtype }}\right)^{2}+ \\
& \sum_{j=1}^{\text {nangles }} k_{j, \text { angletype }}\left(\theta-\theta_{0, \text { angletype }}\right)^{2}+ \\
& \sum_{k=1}^{n \text { torsions }} \sum_{n=1}^{4} \frac{V_{k, n, \text { torstype }}}{2}\left(1-(-1)^{n} \cos \left(n \varphi-\delta_{n}\right)\right)+
\end{array}\right\}
$$

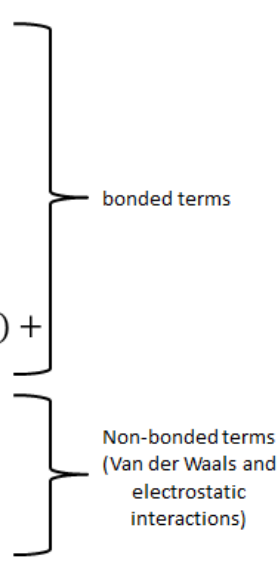

\subsubsection{Molecular mechanics (MM): optimization algorithms}

Optimization algorithms try to search out low-energy conformations for a given initial geometry and FF. In this case, an energy function is minimized as a function of the nuclear coordinates, choosing a given minimization method (e.g. steepest descent, Newton-Raphson, conjugate gradient, simulated annealing etc.). The optimized structures can be used as a starting point for MD or MC simulations.

The main limitations of optimization methods are: i) a lack of convergence from a certain starting point, ii) converging to a stationary point that is not a minimum, iii) generating artificial minima caused by non-bonded truncation cut-offs or non-realistic physical models, and iv) thermal contributions are not taken into account.

In the case of PE, these methods have been used to obtain reasonable structures of amorphous [423] or crystalline [424] states for successive simulations using MD or MC methods.

\subsubsection{Molecular dynamics (MD)}

The MD method is a deterministic technique that simulates the motion of a system under the influence of a given potential [FF, as that shown in Equation (16)] by following molecular conformations according to Newton's equation of motion. The system of equations of motion of $\mathrm{N}$ particles is:

$$
\overrightarrow{\boldsymbol{f}_{\boldsymbol{\imath}}}\left(\overrightarrow{\boldsymbol{r}_{\boldsymbol{\imath}}}\right)=-\nabla V\left(\overrightarrow{\boldsymbol{r}_{\boldsymbol{\imath}}}\right)=-m_{i} \frac{d^{2} \overrightarrow{\boldsymbol{r}_{\boldsymbol{\imath}}}}{d t^{2}}
$$

The time evolution of the particles (trajectory) is obtained by integrating the equations of motion [Equation (17)] over discrete steps in time (time-step, $\Delta \mathrm{t}$ ) using a finite difference method. The time-step must be sufficiently small to avoid discrete integration errors. Typical time-steps in atomistic MD simulations are in the order of 1 fs, thus many millions of steps are needed for a single simulation in the nanoscale. The Verlet algorithm [425] is the most widely used integration scheme due to its simplicity 
and ease of implementation. Using a Taylor expansion, we can calculate the particle's position at a later time, $\mathbf{r}(\mathrm{t}+\Delta \mathrm{t})$, as a function of positions in the current and previous steps $(\mathbf{r}(\mathrm{t})$ and $\mathbf{r}(\mathrm{t}-\Delta \mathrm{t})$, respectively) and accelerations in time for the current step $\mathbf{a}(\mathrm{t})$. Velocities at a later time $\mathbf{v}(\mathrm{t}+\Delta \mathrm{t})$ can be calculated similarly from $\mathbf{r}(\mathrm{t})$ and $\mathbf{r}(\mathrm{t}-\Delta \mathrm{t})$.

By far, MD simulations are the most used computational techniques to study PE properties. Among others, MD simulations have been performed to calculate static equilibrium properties (radius of gyration, end-to-end distances, PVT properties, static structure factor, pair-distribution functions etc.) [47, 48, 53, 58, 61-74, 81, 85, 86, 100, 112], dynamic properties (mean-square displacements, diffusion coefficient, dynamic structure factors, etc.) and rheological properties (intrinsic viscosity, entanglement molecular weight, entanglement relaxation time etc.) [70, 72-74, 76, 142-144, 153]. Crystallization [73, 308, 356] and melting [273, 315, 349, 356, 426] processes for semicrystalline PE have been also simulated using atomistic MD methods. Many of these properties can be directly compared with experimental data, as shown in the preceding sections. Also, comparisons with experimental data can be used to validate the FF used in the simulations [73].

MD equilibrium simulations can be used to study non-equilibrium properties through Green-Kubo formalism [427, 428]. However, this approach is based on the computation of time correlation functions, and thus computing requirements might be larger than the available computational time. Further, non-equilibrium properties may only be addressed in the linear regime, thus remaining close to equilibrium. These limitations have prompted the development of non-equilibrium molecular dynamics (NEMD) algorithms. The basis of NEMD simulations is to produce a non-equilibrium steady state in a molecular system by applying a suitable external field (i.e. planar Couette flow). In the case of polymers, the SLLOD algorithm is particularly useful and allows for NEMD simulations examining rheological properties [180, 429]. This algorithm applies a planar Couette flow field at a strain rate resulting in strain-rate dependent shear-thinning viscosity. Some applications of NEMD to study rheological properties of PE have been discussed in the previous sections [153, 177, 178, 183, 186].

There many available commercial and open-source packages for the MD simulation of polymers, among others those supplied by Materials Studio [430], GROMACS [431], LAMMPS [432] and HOOMD-Blue [433].

\subsubsection{Monte Carlo (MC)}

MC algorithms stochastically generate trial configurations according to the distribution probability of a working statistical ensemble (e.g. NVT, NPT). MC algorithms are composed of a set of MC moves that generate a Markov chain of states. That is, the new configurations are generated with probabilities that depend only on the current state (no history dependence). Each MC move is determined by the transition probability of moving from an old to a new state $\left(\pi_{o \rightarrow n}\right)$, which can be written as:

$$
\pi_{o \rightarrow n}=\alpha_{o \rightarrow n} p_{o \rightarrow n}
$$

where $\alpha_{o \rightarrow n}$ is the conditional probability of a trial move from the old (o) to a new state (n) and $p_{o \rightarrow n}$ is the acceptance probability. Let us denote the equilibrium density probability in the working ensemble of finding the system in new or old states as $\rho_{n}$ or $\rho_{o}$, respectively. The rule that generates $\mathrm{n}$ from o must ensure that an arbitrary initial distribution eventually tends to the stationary (equilibrium) distribution density. Further, if the rule obeys the detailed balance condition: 


$$
\rho_{o} \pi_{o \rightarrow n}=\rho_{n} \pi_{n \rightarrow o}
$$

then an equilibrium process is approached, and the sampling is correct to generate a set of configurations. By substituting Equation 19 in Equation 18, we obtain:

$$
\rho_{o} \alpha_{o \rightarrow n} p_{o \rightarrow n}=\rho_{n} \alpha_{n \rightarrow o} p_{n \rightarrow o}
$$

Applying the asymmetric Metropolis rule [33, 34, 38, 434, 435], the probability of acceptance of a MC move is given by:

$$
p_{o \rightarrow n}=\min \left(1, \frac{\alpha_{n \rightarrow o}}{\alpha_{o \rightarrow n}} \frac{\rho_{n}}{\rho_{o}}\right)
$$

Biased MC moves can be formulated to improve sampling. In these moves, the MC trial move is biased toward reasonable configurations and to approach the right distributions, the conditional probabilities $\left(\alpha_{o \rightarrow n}\right.$ and $\left.\alpha_{n \rightarrow 0}\right)$ need to be subsequently corrected.

For polymers, and in particular for both linear and branched PE, there are a number of MC moves that can be applied to generate the conformations. These can be divided into two major subgroups [436-439]: i) non-altering connectivity moves (flip, concerted rotation, end-chain rotation and configurational bias of end-segment moves), and ii) altering connectivity moves (end-bridge, double bridge etc.). Altering connectivity moves have proved to be efficient for equilibrating long polymer chains and their combination with MD simulations opens the door to calculate rheological properties for industrial-relevant PE [58, 69, 71].

MC simulations are not constrained by the restrictions of solving Newton's equation of motion, and this is their main advantage. This lack of restrictions allows the design of MC moves that generate trial configurations within a given statistical ensemble. Although implementing the moves may be nontrivial, they may lead to high speedups when sampling equilibrium properties. However, as the equation of motions is not solved and moves can alter conformations in a dynamically unphysical way to accelerate sampling, dynamic information cannot be obtained from typical MC simulations. Most importantly, for dense polymer systems such as PE melts, MC moves significantly alter the system's coordinates. This likely turns out in a great overlap of atoms and thus in low acceptance of the trial configurations. Unlike MD simulations, the implementation of MC moves is difficult for general cases and this explains the lack of available software to perform MC simulations on polymer systems. Two remarkable exceptions are Cassandra [440, 441] and MCCCS Towhee [442]. These programmes are open-source MC software for molecules and could represent a good starting point to implement MC moves for polymers, especially PE.

Classically, lattice MC models have been proposed to simulate polymers [33]. Mattice's group has developed a dynamical MC lattice model to study the dynamics and crystallization of polymers. This approach is based on both a high coordination lattice and the rotational isomeric states (RIS) model to calculate the potential energy of the chain [443-446]. Other popular lattice method is the bond fluctuation model (BFM), which is based on a mapping of the real polymer onto a lattice model, where each repeat unit or effective monomer of the lattice is a unit cube on the simple cubic lattice. The Hamiltonian used in the simulation is parametrized using certain geometric and kinetic data of the polymer under study [447]. The BFM model has been applied to study PE systems [230, 448]. 
These models retain sufficient detail to enable distinction of the precise chemical composition of the polymers. Through these approaches have inherent limitations to represent chain motions and conformations, they allow very efficient and fast implementations. Thus, they can contribute to the understanding of the polymer physics, especially where other techniques, as off-lattice MC moves above described, cannot be applied due to its high computational cost or low acceptance ratio.

\subsubsection{The problem of equilibrating long polyethylene chains}

The dynamics of PE (or in general of polymers) can span many decades of time. On one hand, local conformational fluctuations typically occur in the nanosecond regime and are almost independent of the polymer $\mathrm{M}_{\mathrm{w}}$, while on the other hand, the long molecular relaxations (i.e. reptational diffusion of the chain) of entangled PE strongly depend on $\mathrm{M}_{\mathrm{w}}$ and may take place in the order of seconds. Unfortunately, with the currently available computational resources, atomistic MD simulations are limited to times of a few microseconds in the best of the cases. Thus, it is clear that brute-force atomistic MD simulations of high $\mathrm{M}_{\mathrm{w}}$ polymers are out of the question, even to obtain a well-equilibrated system. In extreme cases, most of the computer time is used for the equilibration stage before any useful data can be generated from the simulation.

A number of authors have proposed strategies to speed-up the equilibration stage of relatively high $\mathrm{M}_{\mathrm{w}}$ systems:

- A first strategy consists of so-called hierarchical MC/MD simulations. Firstly, a number of unphysical MC moves (such as the altering connectivity moves discussed in the previous section) are used to accelerate dynamics and provide a well-equilibrated system. Subsequently, these well-equilibrated structures are used as input for relatively short MD simulations, which reproduce real physical polymer dynamics $[64,69,70,72,76,84]$. The main benefit of this strategy is that equilibration is accelerated several order of magnitudes. However, the complex MC moves required can be difficult to implement for non-linear topologies (i.e. SCB or LCB PE systems).

- Algorithms using soft non-bonded potentials and a push-off process [449, 450] allow the chains to overlap and cross each other, accelerating the relaxation process at large length-scales. At the same time, these algorithms should keep connectivity and small- and medium length scales close to equilibrium values [449]. Finally, full non-bonded potentials are recovered and the production simulation can start.

- Sequential back-mapping from a coarse-grained (CG) to a fine-grained (FG) representation until atomistic details are recovered. A first method is that proposed by Zhang et al. [451], starting from a very CG representation of the polymer [452] applied sequentially to introduce details in the model. The method has proved to be computationally efficient since in each step the introduction of details only involves local polymer relaxation; thus it does not depend on the $\mathrm{M}_{\mathrm{w}}$ of the polymer. However, as far as we know, this method has not been applied to obtain a full PE system; rather a CG Kremer-Grest representation is obtained [149]. A second method has been proposed by Carbone et al. [86] for the long length-scale. In this case, the chains are built as a random walk using the characteristic Kuhn length of the polymer under study. Atomistic details are introduced by substituting Kuhn fragments with atomistic ones, and then several MD simulations using soft-core potentials are performed to equilibrate intermediate- and short length scales. Recently, Svaneborg et al. 
proposed an effective multiscale method for equilibrating Kremer-Grest model polymer melts [149]. In this case, the melt structure is progressively equilibrated above the tube scale, inside the tube, and finally recovers details at monomeric scales. Extensions to branched or polydisperse chains are straightforward [453].

- Hybrid AA/SCF is a theoretical scheme for a simulation technique whereby self-consistent field theory (SCF) and MD simulations are concurrent in a molecular model $[454,455]$. These methodologies are more computationally efficient to equilibrate polymer configurations than brute force MD [456]. This is because intermolecular nonbonded forces are replaced by the calculation of interaction forces between independent particles in an average density field.

All these strategies are useful to obtain well-equilibrated PE systems. However, once equilibration is reached, very long MD simulations may still be needed to examine the slow reptation dynamics characteristic of entangled PE. As detailed in the next section, mesoscale and multiscale simulations try to alleviate this problem.

\subsubsection{Polyethylene force fields}

As seen above, FFs are a key ingredient of classic atomistic simulations. Several FFs have been used in the literature to simulate PE-based polymers. The most important characteristics of the typical FFs for PE are the following:

- OPLS (optimized potentials for liquid simulations). The UA [43] and the AA $[457,458]$ versions of this FF have been optimized in Jorgensen's lab by fitting experimental properties such as density and vaporization heat. Torsion potentials are optimized to fit the torsional profiles in gas-phase, generally obtained from QM calculations. In the AA version, electrostatic charges, often from QM calculations, are used to obtain Coulombic forces. A recent version used for PE [95] is the optimization of parameters for long chain n-alkanes [459].

- PYS-UA (Paul-Yoon-Smith United Atom) [46] and PYS-AA [39, 40]. This FF was optimized to study long PE chains. The original version uses a constrained potential for bond length $(\mathrm{C}-\mathrm{C}=1.54 \AA$ ) and a harmonic potential to reproduce the $\mathrm{C}-\mathrm{C}-\mathrm{C}$ angle of $110^{\circ}$. Torsion parameters are based on Raman experiments of pentane. Non-bonded $\mathrm{CH}_{3}$ and $\mathrm{CH}_{2}$ are modelled using the same LennardJones parameters. Electrostatic potential is not used in this FF. An AA version has been also optimized and used to simulate PE [39, 40].

- HMT-UA (Harmandaris-Mavrantzas-Theodorou). These authors use a FF, borrowing parameters from different sources [460-462]. In their simulations, a successful description of conformational and dynamic properties was obtained, including crossover between the Rouse and reptation regimes [47, 48, 58, 8284].

- SKS-UA (Siepmann-Karborni-Smit) [44]. This FF was created to reproduce the vapour-liquid equilibrium of alkanes of different length. It can be considered a primitive version of the TraPPE-UA FF.

- TraPPE-UA (TRAnsferable Potentials for Phase Equilibria) [50, 51]. This FF was developed by Siepmann's group. Bonded, angle and dihedral interactions are fitted using either experimental data or QM calculations. Then, the nonbonded potentials of representative simple molecules are found through empirical fitting to reproduce experimental phase equilibrium data. TraPPE-UA 
arranges transferability and accuracy in the calculations of phase equilibria and thermophysical properties. MC and MD simulations using TraPPE-UA have been widely used for the study of static and dynamic properties of n-alkanes and PE melts, including the impacts of molecular length [81], miscibility [100], molecular architecture in tri-arm stars, H-shaped and pompom [66-68], SCB [61, $67-69,71,72,74]$ and basic properties of the entangled nature of PE [70, 73], as seen in the previous sections.

- TraPPE-EH (TRAnsferable Potentials for Phase Equilibria-Extended Hydrogen) [41]. It is an explicit-hydrogen model for alkanes. This explicit representation yields considerably better agreement with experimental results that the UA version for short alkanes, but the price to pay is a higher computational cost, which limits its applicability for high-molecular weight alkanes. The performance of other models, such as TraPPE-UA, OPLS-AA and OPLS-UA, to calculate some thermodynamics properties has been compared with the TraPPEEH model [41].

- NERD-UA (revised by Nath-Escobedo-de Pablo) [49]. This FF is similar to TraPPE-UA and SKS-UA FFs.

- KMT-UA (Karayiannis-Mavrantzas-Theodorou). This FF is a modified TraPPEUA FF using a different torsion expression based on the Toxvaerd potential [463]. This FF has been used in the study of n-alkanes, linear [53, 62-64], LCB [75, 76], SCB [77] and cyclic ("ring") [65] PE.

- Dreiding II. It is a generic force field for predicting structures and dynamics of organic, biological, and main-group inorganic molecules [464]. This FF has been used to study the folding of PE chains [267-271, 279, 280, 282]. This force field has a flat gauche well and a cis barrier too high in comparison with other force fields [465]. This leads to a very rigid model predicting a persistence length of $50 \AA$ [267] in comparison with the $6 \AA$ obtained in SANS experiments [108]. For this reason, this FF has been criticized as unrealistic, in comparison with others of similar computational cost, such as PYS-UA [319].

- COMPASS (Condensed-Phase optimized molecular potentials for atomistic simulation studies) [466]. This is an explicit hydrogen force field. It was parametrized using quantum-molecular calculations enabling a reliable prediction of the structural, conformational and thermophysical properties for a broad range of molecules and polymers in condensed phase. However, its complexity (i.e., cross terms) in comparison to the classical force fields limited its applicability to PE long chains, due to its high computational cost.

It should be noted that UA-FFs and CG models have some limitations to simulate the crystal phase of polymers in comparison with the explicit hydrogen FFs [254, 467, 468]. For example, it is well known that UA-FFs favour the hexagonal crystal phase of PE over the more commonly observed orthorhombic crystal phase [233, 468]. However, FFs such as PYS-UA [73, 233], TraPPE-UA [73] and CG potentials derived from IBI $[355,356]$ have shown to be realistic studying the early stages of the crystallization process of PE.

\subsection{Mesoscale models}

Many spatial and dynamic phenomena in polymer materials (in particular PE) take place in length-time scales which cannot be directly addressed by conventional MD based on FFs. The mesoscopic regime covers intermediate scales between the AA 
simulations depending on the chemical details and the continuum representation that characterizes the thermodynamic properties of the material. As an example, the full dynamics of entangled PE materials from sub-diffusive local motions, passing through segment motions between entanglements, to the full reptation of chains cannot be captured using atomistic MD simulations alone. Simulations that capture the whole dynamic regime are of utmost importance to understand and predict both the rheological and mechanical properties of PE-based materials.

In general, mesoscale methods are based on statistical mechanics, whose purpose is to establish a link between the microscopy description and macroscopic thermodynamic properties. In practice, these methods are based on a CG process of averaging out "nonrelevant" microscopy degrees of freedom, which can be attained by lumping atoms into larger particles (known as mapping process), thereby reducing the number of degrees of freedom used to describe the system. After this mapping, an effective potential that acts between the CG particles is needed. Further, it is important to note that these CG potentials depending on temperature, density and pressure, among other factors, are often due to the average-out process in the mesoscale $[469,470]$.

The theory underlying CG dynamics is the Mori-Zwanzig formalism, whereby a projection operator technique leads to a generalized Langevin equation (GLE) governing the dynamics of the coarse variables. The GLE is stochastic in nature and includes memory and dissipation (friction) effects, which need to be taken into account during the CG process (please see reference [32] for details). In the following subsections, we offer a succinct explanation of various mesoscopic methods covering the intermediate length-time scales linking atomistic and macroscale techniques. Of the numerous existing mesoscale methods, we have selected those used to simulate PEbased materials. Interested readers are referred to the excellent reviews written by Padding et al. [32] and Guenza [471].

In general, in a particle-based approach, a number of atoms are lumped or grouped on a super-atom particle or CG bead particle in a process called "mapping". This number is known as the degree of CG, $\lambda$. Depending on both, the mapping method and $\lambda$, an effective interaction potential or potential of mean force (PMF) of variable softness is obtained. Next, the time evolution of the system is followed using the appropriate equation of motions, which fundamentally depends on the softness of the PMF.

\subsubsection{Coarse-grained molecular dynamics (CGMD)}

The softness or hardness of the interaction between CG sites depends on the number of atoms, $\lambda$, lumped onto a CG bead. The higher the number of atoms grouped in a CG bead the softer the PMF between beads will be. Averaging-out degrees of freedom leads to smoothened free-energy surfaces, which can be offset using friction and random forces. However, the precise treatment of dissipation requires a sophisticated generalized Langevin equation [32]. It is well-known that ignoring this dissipation leads to a faster dynamics compared to either atomistic or experimental reference data. This is usually beneficial for the equilibration process, but requires rescaling of the time scales when a quantitative comparison with real dynamics is desired. Furthermore, it is assumed that the number and nature of entanglements are conserved under these conditions of low $\lambda$ values, whereby the relatively hard PMF avoids unphysical overlapping and crossing of bonds between different chains.

CGMD methods can be classified into two groups (see references therein in [472]): i) force matching methods, and ii) structure-based methods (e.g. iterative Boltzmann 
inversion (IBI), inverse MC and Newton inversion methods). The force matching method employs a variation procedure to deduce effective $\mathrm{CG}$ interactions directly from the force information obtained from atomically detailed simulations. To the best of our knowledge these methods have not been used for PE. In contrast, structure-based methods construct the CG potential to reproduce a structural property calculated from short AA simulations such as angle distributions or pair distribution functions. By far, IBI is the most used method in the case of polymers.

Boltzmann inversion (BI) reads as $U_{P M F}^{C G}(b, \theta, \varphi, r)=k_{B} T \exp (-P(b, \theta, \varphi, r))$, where $P(b, \theta, \varphi, r)$ is the total probability distribution function. In practical terms, it is assumed that all probability distributions are independent, that is $P(b, \theta, \varphi, r)=$ $P(b) P(\theta) P(\varphi) P(r)$. This assumption needs to be confirmed "a posteriori" and in the case of dependence between probabilities, corrections are needed. Each of the distributions is calculated by the normalized histogram obtained from AA simulations of the bond, angle and torsion distributions for the bonded interactions and of the pair distribution function for the non-bonded interactions. These histograms are the target distributions in the CG process. Frequently, one simple BI is not sufficient to match the target distributions, then an iterative algorithm is run in which each iteration improves the calculated CG potential to reproduce the target distribution according to:

$$
U_{P M F, i+1}^{C G}=U_{P M F, i}^{C G}+\alpha k_{B} T \frac{P_{i}(q)}{P_{\text {target }}(q)}, \text { where } q \in\{b, \theta, \varphi, r\}
$$

,parameter $\alpha$ is a relaxation factor between 0 and 1, which helps stabilize the numerical scheme.

Chen et al. used a CG degree of 8 monomers ( $16 \mathrm{CH}_{2}$ units) per single bead, noting that, even for entangled PE, Rouse rather than reptation dynamics was obtained in the simulation. This is due to the unphysical crossing of chains of the soft CG potential used for $\lambda=16$ [473]. Recently, Salerno et al. [94, 95] systematically studied the CG level reproducing the dynamics of PE. These authors found that up to two monomers ( $\lambda$ =4) can be lumped into a single CG bead to qualitatively describe the dynamic regimes characteristic of long entangled PE chains. Quantitative agreement is reached applying a time-scaling process to all atomistic reference data. In contrast, when three monomers $(\lambda=6)$ are grouped in a single CG bead, topological constraints are not conserved, that is, the chains cross each other giving rise to an unphysical dynamics. To avoid this and keep the topological constraints, an algorithm needs to be implemented [474].

Finally, Carbone et al. [469] observed that polymer chain length does not affect the temperature transferability of the CG PMF. However, the $\lambda$ value is critical as its increase causes a sharp decrease in the temperature's transferability. Thus special care should be taken choosing this value. In the case of PE, Vettorel et al. [355] claimed that as a first approximation, $C G$ potentials can be used with $\lambda=2$ mapped at a single temperature from an all-atom simulation but only when using iteratively optimized models as shown in their paper. Fukunaga et al. reported that the CG model $\lambda=3$ reproduces the radii of gyration and various distribution functions of the $\mathrm{CG}$ variables over a wide temperature range [475].

\subsubsection{Ultra coarse-grained stochastic dynamics (CGSD)}

When $\lambda$ is sufficiently large, other techniques need to be considered as effective potentials start to become very soft. A value of $\lambda \geq 10$ backbone units in polymers has been suggested by Padding and Briels [32]. Under this condition, friction and stochastic 
forces are so important that they cannot be simply ignored as done in the previous section. The lack of treatment of both friction and stochastic forces in those soft potential models cannot prevent unphysical overlapping and crossing of atoms during simulation thus leading to unreal dynamics. Next, these forces need to be explicitly treated in the equation of motions.

When treating friction and stochastic forces, we may be met with significant complications. Firstly, following the theory of Mori-Zwanzig, a GLE can be formulated introducing friction and random terms. However, at least three problems need to be addressed from the simulation's perspective: i) the GLE has a memory kernel and it is not known how to generate it, ii) this force depends on the position and velocity of all particles, and iii) the solutions to GLE are difficult to implement numerically. Usually, these problems are overcome by assuming a pairwise additive friction coefficient between two CG beads and using a Markov approximation (no memory effects) of the time dependence of the friction. These approximations need to be carefully tested in each specific system.

Padding et al. examined melt dynamics using CG models derived from AA trajectories of short linear PE chains $\left(\mathrm{C}_{120} \mathrm{H}_{240}\right)$ as a reference $[89,476]$. A value of $\lambda=$ $20 \mathrm{CH}_{2}$ units was chosen, which is roughly $1 / 3$ of the smallest entanglement length of PE [22]. The effective PMF was calculated by fitting analytical Gaussian functions to the AA distribution functions between nonbonded and bonded centres of mass. In order to avoid the unphysical crossing of chains in the CG model, the authors introduced an uncrossability constraint in the so-called TWENTANGLEMENT algorithm. A range of PE melts from $\mathrm{C}_{80}$ to $\mathrm{C}_{1000}$ ( $\mathrm{B}_{4}$ and $\mathrm{B}_{50}$ in $\mathrm{CG}$ representation) in chain length were explored using this algorithm and it was concluded that the $\mathrm{CG}$ model can reproduce Rouse and reptation dynamics as a function of the chain length [90]. NEMD simulations using this CG model have also been used to investigate the transient and steady-state nonlinear flow properties of unentangled and moderately entangled PE melts, finding a good agreement with experiments [192]. Rheological properties of complex molecular architectures such as entangled star PE melts have been analyzed using this model and results were compared with those of their linear counterparts [477].

Guenza's group introduced a modelling approach based on the Ornstein-Zernike (OZ) equations, in which the whole polymer chain is defined as a CG soft colloidal particle of size in the scale of its radius of gyration [478]. By combining this method with the PRISM scheme [479], there seems to be thermodynamic consistency [480]. However, there is some debate concerning this thermodynamic consistency [481, 482]. In later work, appropriate scaling of the artificially fast dynamics of simulated CG polymer melts to provide realistic values was proposed [483]. This model has shown its ability to properly capture the static structural properties of PE (i.e. pair-distribution functions). In parallel, the model quantitatively agrees with the dynamic properties of untangled and moderately entangled PE models $\left(\mathrm{C}_{30}\right.$ and $\left.\mathrm{C}_{320}\right)$ at large scales, both those offered by experiments or atomistic simulations. However, rheological properties have not yet been calculated using this model.

Briels' group developed a particle based CG model of polymer melts in which each polymer chain is represented as just one particle. This model is able to reproduce both the static and dynamic properties of a PE melt $\left(\mathrm{C}_{800}\right)[484,485]$.

\subsubsection{Dissipative particle dynamics (DPD)}

The dissipative particle dynamics (DPD) method was formerly proposed by Hoogerbrugge and Koelman to describe incompressible complex fluids [486]. 
Subsequently the procedure was modified by Español and Warren [487] and Groot and Warren [488] to accomplish the fluctuation-dissipation theorem and link the atomistic scale to the mesoscale using Flory-Huggins parameters $(\chi)$ obtained in small-sized model systems simulated at atomistic scale. The time evolution of the particles is given by Newton's equations of motion, and the total force acting on a particle is expressed as the sum of pairwise repulsive conservative $\left(F_{i j}^{C}\right)$, dissipative $\left(F_{i j}^{D}\right)$, and random $\left(F_{i j}^{R}\right)$ forces, respectively. The $F_{i j}^{D}$ term represents the effects of viscosity and slows down the particles' motion with respect to each other, whereas $F_{i j}^{R}$ represents the thermal or vibrational energy of the system. DPD simulations, unlike Brownian dynamics, offer correct hydrodynamic behaviour.

DPD simulations have been widely applied to study the phase diagrams of blends of PE with other polymers [489-491] and PE with nanocomposites [492, 493]. These studies focus on the final states as a function of temperature and blend composition, thus the accelerated dynamics of DPD (due to unphysical overlap and crossing of particles) offers an advantage and dynamic properties are not studied at all. Notwithstanding, some authors have used DPD simulations to obtain the equilibrium and dynamic properties of PE. Rousseau's group in collaboration with Theodorou's group have published several studies of DPD simulations of PE properties [494-497]. These groups have examined both the effect of the degree of CG $(\lambda=6-20)$ and length of the $\mathrm{CG}$ chain $\left(\mathrm{N}=6-50\right.$ beads) on end-to-end distances $\left(\mathrm{R}_{\text {ete }}\right)$, diffusion coefficients of the polymer chain, and relaxation time of the end-to-end vectors. They also observed good agreement between the equilibrium properties (radial pair distribution functions and $\mathrm{R}_{\text {ete }}$ ) and simulations performed at the atomistic level.

However, they did not observe cross-over from Rousse to reptation. Because of the high CG level used, bead-bead intermolecular interactions are much softer than Lennard-Jones interactions, and beads can easily overlap. As a consequence, unphysical bond crossing may occur, the topological constraints imposed by chain uncrossability can disappear, and this may prevent the formation of entanglements. To avoid this problem, a segmental restraint algorithm based on the Kumar and Larson model [498] was proposed. Using this algorithm for long chain systems, the global dynamics typical of reptation were recovered and rheological properties were monitored as a function of $\mathrm{M}_{\mathrm{w}}$. Other segmental repulsive force methods to avoid bond-crossings have been proposed [474, 499, 500]. The main problems with these approaches are: i) they are computationally expensive, and ii) some parameters in the algorithms are physically unclear. The algorithm proposed by Sirk et al. has been recently used by Salerno et al. [94] to examine the rheological properties of PE. Nikunen et al. [501] have proposed a method, which introduces simple topological constraints to prevent bond-crossings. Using this approach, crossover between Rouse for short chains and reptational dynamics for long chains were recovered. Although, the model has been successfully applied in some simulations [502, 503], it has recently shown some deficiencies when applied to systems with many DPD beads, which could limit its application to PE systems [504]. A highly CG model has been proposed by Zhu et al. [505] to simulate the entangled polymer melts. In this model, a polymer chain is considered as a single CG particle, and the creation and annihilation of entanglements are regarded as stochastic events in proper time intervals according to certain rules and possibilities. The authors applied this model to simulate the $\mathrm{C}_{1000} \mathrm{H}_{2002}$ system at $450 \mathrm{~K}$, validating the model by comparing results to experimental data and to other simulation results. 


\subsection{Multiscale modelling of polymers}

As shown in the previous sections, polymer materials span their behaviour across a very large length-time scale. Thus, no single computational tool is able to encompass the physics and chemistry that govern the properties of PE-based materials on these diverse scales. Therefore, depending on the physical process under study, it is necessary to choose the suitable computational method capable of describing the polymer at a given scale. Figure 18 shows a representation of the hierarchical organization of computational methods commonly used in polymer modelling.

The bottom-up approach uses methods from a detailed length-time scale to calculate parameters which can be used as inputs in a coarser scale. In contrast, the top-down view uses macroscopic properties to parametrize models at a lower scale. As a typical example, a FF for atomistic simulations could be obtained from methods arising from smaller scales such as QM following a bottom-up approach. Also, thermodynamics properties such as density or heat of vaporization can be obtained from an equation of state in a top-down approach to capture the parameters for the FF.

The holy grail of multiscale simulations for polymers according to very basic principles should be able to: i) understand fundamental mechanics, such as assembly or crystallization processes, and ii) predict the properties of new polymers to design new materials.

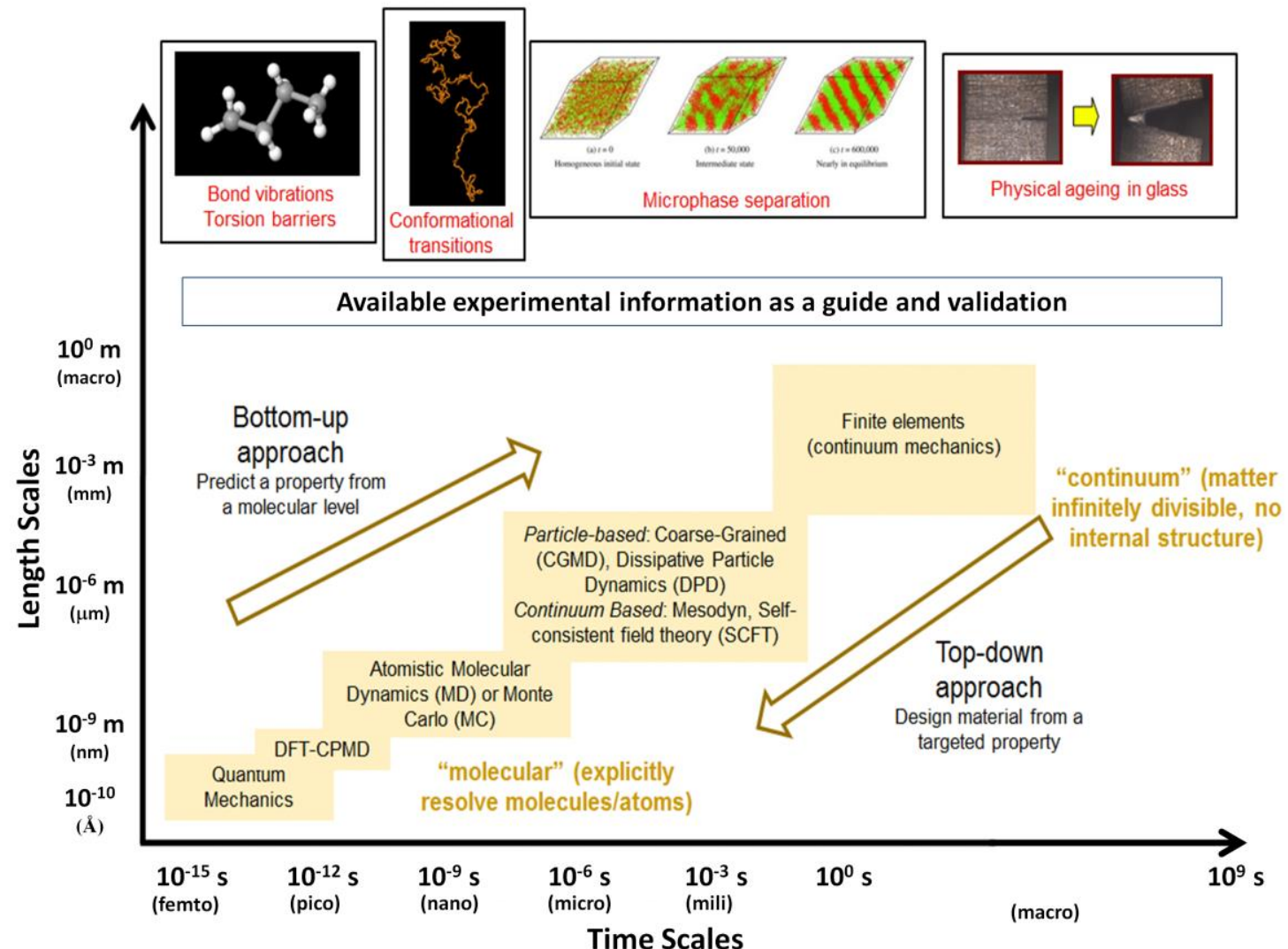

Figure 18. Multiscale view of polymer modelling.

\subsubsection{Concurrent coupling}

Concurrent coupling makes reference to link two (or more) different scale methods (i.e. atomistic MD and CGMD) within a single simulation. For a concurrent simulation, a spatial decomposition of the domain between the two methods needs to be formulated. These domains have different resolution and accuracy. For example, atomistic 
simulation is performed in a localized region where atomistic details are driven by specific chemical-physical interactions, and a CG resolution is used for the remaining regions. The great challenge of concurrent simulations is treatment of the linking area (aka handshakes regions) between the two scales.

Initial models do not allow for changing region resolutions during the simulation, since there is no mass transfer between scales. More sophisticated techniques, in which onthe-fly changes of resolution are allowed, have been recently proposed [506-510] [493497].

Simulations of PE using concurrent schemes are rather scarce. Nielsen et al. [509] considers coupling between the different resolutions to study the swelling of a PE chain in a theta solvent (hexane). Di Pasquale et al. [511] performed simulations of PE melt models using a hybrid model in which a mixture of AA and CG particles (AA-CG) are present in a polymer chain, for which the CG potential was obtained using the IBI procedure. The chain size and structural properties of the AA-CG PE melt are well reproduced when compared with the AA PE model, with the benefit that is less computationally demanding.

\subsubsection{Hierarchical or sequential coupling}

In hierarchical multiscale modelling, the information obtained at a level of description is used in other scales by integrating sequentially simulation techniques on different length-time scales. The bottom-up approach passes information from a more detailed scale to a less detailed scale, whereas the top-down approach goes from less to more detailed levels $[29,30]$.

In the case of PE, the most usual simulations are those that combine AA and CG simulations in a hierarchical way. At the AA level, a small system is simulated to obtain inter- or intramolecular distributions, which are used to generate mapping between AA and CG models under an effective potential. These kinds of simulation have been used to study crystallization processes [356] and structural and dynamic properties in the melt [70], among others [29, 30], as widely covered in the previous sections.

\section{Concluding remarks}

As an overall conclusion, computer simulation methodologies have reached such a level of development that we can now feasibly address real experiments in polymer systems. As an example, it was only recently that the entanglement relaxation time was directly determined experimentally using an advanced rheological instrument. The experimental result was in very good agreement with the one predicted by computer simulations.

In this review of the literature on experiments and computation, we find agreement between the length and time scales offered by both approaches. Thus, computational methods are emerging as powerful tools both to assess physical experimental properties and to drive the design of new fundamental experiments.

In the case of polyethylene, the results provided so far by computational methods are providing direction for future studies designed to examine more complex molecular architectures and even as yet non-synthesized materials such as, for example, ring polyethylene. 


\section{Acknowledgments}

This research work was supported by the CSIC (Spain) - Project PIE201360E097. J. Ramos acknowledges financial support through the Ramón y Cajal programme (MINECO, Spain) - Contract RYC-2011-09585. We are indebted to the Spanish oil company Repsol for close collaboration over many years of research into the synthesis and properties of polyolefins and rubbers. 
[1] http://www.essentialchemicalindustry.org/polymers/polyethene.html. 2017 (accessed 410-2017.2017).

[2] G. Ungar, X.-b. Zeng, Learning Polymer Crystallization with the Aid of Linear, Branched and Cyclic Model Compounds, Chemical Reviews 101(12) (2001) 4157-4188.

[3] V.L. Cruz, A. Muñoz-Escalona, J. Martinez-Salazar, Ab initio calculation of ethylene insertion in zirconocene catalyst systems: A comparative study between bridged and unbridged complexes, Polymer 37(9) (1996) 1663-1667.

[4] J. Ramos, V. Cruz, A. Muñoz-Escalona, J. Martínez-Salazar, Ab initio study of hydrogenolysis as a chain transfer mechanism in olefin polymerization catalyzed by metallocenes, Polymer 41(16) (2000) 6161-6169.

[5] J. Ramos, V. Cruz, A. Muñoz-Escalona, J. Martínez-Salazar, Computational studies of the Brookhart's type catalysts for ethylene polymerization. 1. Effect of the active site conformations on the catalyst activities, Polymer 42(19) (2001) 8019-8023.

[6] J. Ramos, V. Cruz, A. Muñoz-Escalona, J. Martínez-Salazar, A computational study of ironbased Gibson-Brookhart catalysts for the copolymerisation of ethylene and 1-hexene, Polymer 43(13) (2002) 3635-3645.

[7] J. Ramos, V. Cruz, A. Muñoz-Escalona, S. Martínez, J. Martínez-Salazar, Computational studies of the Brookhart's type catalysts for ethylene polymerisation. Part 2: ethylene insertion and chain transfer mechanisms, Polymer 44(7) (2003) 2169-2176.

[8] J. Ramos, A. Muñoz-Escalona, V. Cruz, J. Martínez-Salazar, DFT study of hydrogenolysis as a chain transfer mechanism in olefin polymerisation catalysed by nickel-diimine-type catalysts, Polymer 44(7) (2003) 2177-2186.

[9] V. Cruz, J. Ramos, A. Muñoz-Escalona, P. Lafuente, B. Peña, J. Martinez-Salazar, 3D-QSAR analysis of metallocene-based catalysts used in ethylene polymerisation, Polymer 45(6) (2004) 2061-2072.

[10] V.L. Cruz, S. Martinez, J. Martinez-Salazar, D. Polo-Cerón, S. Gómez-Ruiz, M. Fajardo, S. Prashar, 3D-QSAR study of ansa-metallocene catalytic behavior in ethylene polymerization, Polymer 48(16) (2007) 4663-4674.

[11] A. Muñoz-Escalona, J. Ramos, V. Cruz, J. Martínez-Salazar, Effect of a second ethylene molecule on the insertion of ethylene in zirconocene catalyst systems: A QM semiempirical study, Journal of Polymer Science Part A: Polymer Chemistry 38(3) (2000) 571-582.

[12] V.L. Cruz, A. Muñoz-Escalona, J. Martinez-Salazar, A theoretical study of the comonomer effect in the ethylene polymerization with zirconocene catalytic systems, Journal of Polymer Science Part A: Polymer Chemistry 36(7) (1998) 1157-1167.

[13] V.L. Cruz, J. Ramos, S. Martinez, A. Muñoz-Escalona, J. Martinez-Salazar, Structure-Activity Relationship Study of the Metallocene Catalyst Activity in Ethylene Polymerization, Organometallics 24(21) (2005) 5095-5102.

[14] V.L. Cruz, S. Martínez, J. Martínez-Salazar, J. Sancho, Proposed Polymerization Termination Mechanism for 3-R-Indenyl ansa-Zirconocenes $(\mathrm{R}=\mathrm{n}$-Alkyl) Based on DFT Calculations and Experimental Observations, Macromolecules 40(20) (2007) 7413-7415.

[15] J. Martínez, V. Cruz, J. Ramos, S. Gutiérrez-Oliva, J. Martínez-Salazar, A. Toro-Labbé, On the Nature of the Active Site in bis(imino)Pyridyl Iron, a Catalyst for Olefin Polymerization, The Journal of Physical Chemistry C 112(13) (2008) 5023-5028.

[16] S. Martínez, V.L. Cruz, J. Ramos, J. Martínez-Salazar, Polymerization Activity Prediction of Zirconocene Single-Site Catalysts Using 3D Quantitative Structure-Activity Relationship Modeling, Organometallics 31(5) (2012) 1673-1679.

[17] J. Ramos, V.L. Cruz, J. Martínez-Salazar, M. Brasse, P. Palma, J. Campora, Density functional study for the polymerization of ethylene monomer using a new nickel catalyst, Journal of Polymer Science Part A: Polymer Chemistry 48(5) (2010) 1160-1165.

[18] V.L. Cruz, S. Martinez, J. Ramos, J. Martinez-Salazar, 3D-QSAR as a Tool for Understanding and Improving Single-Site Polymerization Catalysts. A Review, Organometallics 33(12) (2014) 2944-2959. 
[19] V.L. Cruz, J. Ramos, J. Martínez-Salazar, S. Gutiérrez-Oliva, A. Toro-Labbé, Theoretical Study on a Multicenter Model Based on Different Metal Oxidation States for the Bis(imino)pyridine Iron Catalysts in Ethylene Polymerization, Organometallics 28(20) (2009) 5889-5895.

[20] J.F. Vega, M. Aguilar, J. Martínez-Salazar, Model linear metallocene-catalyzed polyolefins: Melt rheological behavior and molecular dynamics, Journal of Rheology 47(6) (2003) 15051521.

[21] J.F. Vega, D. Polo-Cerón, S. Gómez-Ruíz, S. Prashar, M. Fajardo, J. Martínez-Salazar, Viscoelasticity and macromolecular topology in single-site catalyzed polyethylene, Journal of Materials Science 43(5) (2008) 1745-1748.

[22] J.M. Carella, W.W. Graessley, L.J. Fetters, Effects of chain microstructure on the viscoelastic properties of linear polymer melts: polybutadienes and hydrogenated polybutadienes, Macromolecules 17(12) (1984) 2775-2786.

[23] L.J. Fetters, W.W. Graessley, R. Krishnamoorti, D.J. Lohse, Melt Chain Dimensions of Poly(ethylene-1-butene) Copolymers via Small Angle Neutron Scattering, Macromolecules 30(17) (1997) 4973-4977.

[24] H. Rachapudy, G.G. Smith, V.R. Raju, W.W. Graessley, Properties of amorphous and crystallizable hydrocarbon polymers. III. Studies of the hydrogenation of polybutadiene, Journal of Polymer Science: Polymer Physics Edition 17(7) (1979) 1211-1222.

[25] G.C. Berry, T.G. Fox, The viscosity of polymers and their concentrated solutions, Fortschritte der Hochpolymeren-Forschung, Springer Berlin Heidelberg, Berlin, Heidelberg, 1968, pp. 261-357.

[26] W.W. Graessley, Effect of long branches on the flow properties of polymers, Accounts of Chemical Research 10(9) (1977) 332-339.

[27] J.M. Carella, J.T. Gotro, W.W. Graessley, Thermorheological effects of long-chain branching in entangled polymer melts, Macromolecules 19(3) (1986) 659-667.

[28] J.F. Vega, J. Martínez-Salazar, Rheological features and molecular architecture of polyethylenes, Polymer Bulletin 50(3) (2003) 197-204.

[29] Y. Li, B. Abberton, M. Kröger, W. Liu, Challenges in Multiscale Modeling of Polymer Dynamics, Polymers 5(2) (2013) 751.

[30] A. Karatrantos, N. Clarke, M. Kröger, Modeling of Polymer Structure and Conformations in Polymer Nanocomposites from Atomistic to Mesoscale: A Review, Polymer Reviews 56(3) (2016) 385-428.

[31] C. Tzoumanekas, D.N. Theodorou, From atomistic simulations to slip-link models of entangled polymer melts: Hierarchical strategies for the prediction of rheological properties, Current Opinion in Solid State and Materials Science 10(2) (2006) 61-72.

[32] J.T. Padding, W.J. Briels, Systematic coarse-graining of the dynamics of entangled polymer melts: the road from chemistry to rheology, Journal of Physics: Condensed Matter 23(23) (2011) 233101.

[33] K. Binder, Monte Carlo and molecular dynamics simulations in polymer science, Oxford University Press, New York 1995.

[34] D.N.Theodorou, M.Kotelyanskii, Simulation Methods for polymer, Second ed., Marcel Dekker, Inc 2004.

[35] M.Laso, E.A.Perpete, Multiscale Modelling of Polymer Properties, First ed., Elsevier 2006. [36] P.D.Gujrati, A.I.Leonov, Modeling and Simulation in Polymers, Wiley-VCH, Weinheim 2010. [37] V. Mittal, Modeling and predicition of polymer nanocomposite properties, Wiley-VCH, Weinheim, Germany 2013.

[38] J.A.f.C. Innovation, Computer simulation of polymeric materials, Springer, Singapore 2016. [39] G.D. Smith, D.Y. Yoon, Equilibrium and dynamic properties of polymethylene melts from molecular dynamics simulations. I. n-Tridecane, The Journal of Chemical Physics 100(1) (1994) 649-658. 
[40] W. Paul, G.D. Smith, D.Y. Yoon, Static and Dynamic Properties of a n-C100H202 Melt from Molecular Dynamics Simulations, Macromolecules 30(25) (1997) 7772-7780.

[41] B. Chen, J.I. Siepmann, Transferable Potentials for Phase Equilibria. 3. Explicit-Hydrogen Description of Normal Alkanes, The Journal of Physical Chemistry B 103(25) (1999) 5370-5379. [42] D.Y. Yoon, G.D. Smith, T. Matsuda, A comparison of a united atom and an explicit atom model in simulations of polymethylene, The Journal of Chemical Physics 98(12) (1993) 1003710043.

[43] W.L. Jorgensen, J.D. Madura, C.J. Swenson, Optimized intermolecular potential functions for liquid hydrocarbons, Journal of the American Chemical Society 106(22) (1984) 6638-6646. [44] J.I. Siepmann, S. Karaborni, B. Smit, Simulating the critical behaviour of complex fluids, Nature 365(6444) (1993) 330-332.

[45] B. Smit, S. Karaborni, J.I. Siepmann, Computer simulations of vapor-liquid phase equilibria of n-alkanes, The Journal of Chemical Physics 102(5) (1995) 2126-2140.

[46] W. Paul, D.Y. Yoon, G.D. Smith, An optimized united atom model for simulations of polymethylene melts, The Journal of Chemical Physics 103(4) (1995) 1702-1709.

[47] V.G. Mavrantzas, D.N. Theodorou, Atomistic Simulation of Polymer Melt Elasticity: Calculation of the Free Energy of an Oriented Polymer Melt, Macromolecules 31(18) (1998) 6310-6332.

[48] V.A. Harmandaris, V.G. Mavrantzas, D.N. Theodorou, Atomistic Molecular Dynamics Simulation of Polydisperse Linear Polyethylene Melts, Macromolecules 31(22) (1998) 79347943.

[49] S.K. Nath, F.A. Escobedo, J.J. de Pablo, On the simulation of vapor-liquid equilibria for alkanes, The Journal of Chemical Physics 108(23) (1998) 9905-9911.

[50] M.G. Martin, J.I. Siepmann, Transferable Potentials for Phase Equilibria. 1. United-Atom Description of n-Alkanes, The Journal of Physical Chemistry B 102(14) (1998) 2569-2577.

[51] M.G. Martin, J.I. Siepmann, Novel Configurational-Bias Monte Carlo Method for Branched Molecules. Transferable Potentials for Phase Equilibria. 2. United-Atom Description of Branched Alkanes, The Journal of Physical Chemistry B 103(21) (1999) 4508-4517.

[52] L.D. Schuler, X. Daura, W.F. van Gunsteren, An improved GROMOS96 force field for aliphatic hydrocarbons in the condensed phase, Journal of Computational Chemistry 22(11) (2001) 1205-1218.

[53] N.C. Karayiannis, V.G. Mavrantzas, D.N. Theodorou, A Novel Monte Carlo Scheme for the Rapid Equilibration of Atomistic Model Polymer Systems of Precisely Defined Molecular Architecture, Physical Review Letters 88(10) (2002) 105503.

[54] T.G. Fox, S. Loshaek, Influence of molecular weight and degree of crosslinking on the specific volume and glass temperature of polymers, Journal of Polymer Science 15(80) (1955) 371-390.

[55] G.T. Dee, T. Ougizawa, D.J. Walsh, The pressure-volume-temperature properties of polyethylene, poly(dimethyl siloxane), poly(ethylene glycol) and poly(propylene glycol) as a function of molecular weight, Polymer 33(16) (1992) 3462-3469.

[56] S.J. Han, D.J. Lohse, P.D. Condo, L.H. Sperling, Pressure-Volume-Temperature properties of polyolefin liquids and their melt miscibility, Journal of Polymer Science Part B: Polymer Physics 37(20) (1999) 2835-2844.

[57] D.S. Pearson, G. Ver Strate, E. Von Meerwall, F.C. Schilling, Viscosity and self-diffusion coefficient of linear polyethylene, Macromolecules 20(5) (1987) 1133-1141.

[58] V.G. Mavrantzas, T.D. Boone, E. Zervopoulou, D.N. Theodorou, End-Bridging Monte Carlo: A Fast Algorithm for Atomistic Simulation of Condensed Phases of Long Polymer Chains, Macromolecules 32(15) (1999) 5072-5096.

[59] K. Hur, R.G. Winkler, D.Y. Yoon, Comparison of Ring and Linear Polyethylene from Molecular Dynamics Simulations, Macromolecules 39(12) (2006) 3975-3977. 
[60] K. Hur, C. Jeong, R.G. Winkler, N. Lacevic, R.H. Gee, D.Y. Yoon, Chain Dynamics of Ring and Linear Polyethylene Melts from Molecular Dynamics Simulations, Macromolecules 44(7) (2011) 2311-2315.

[61] E.K. Boland, J. Liu, J.K. Maranas, A molecular picture of motion in polyolefins, The Journal of Chemical Physics 132(14) (2010) 144901.

[62] N.C. Karayiannis, A.E. Giannousaki, V.G. Mavrantzas, D.N. Theodorou, Atomistic Monte Carlo simulation of strictly monodisperse long polyethylene melts through a generalized chain bridging algorithm, The Journal of Chemical Physics 117(11) (2002) 5465-5479.

[63] K. Foteinopoulou, N.C. Karayiannis, V.G. Mavrantzas, M. Kröger, Primitive Path Identification and Entanglement Statistics in Polymer Melts: Results from Direct Topological Analysis on Atomistic Polyethylene Models, Macromolecules 39(12) (2006) 4207-4216.

[64] K. Foteinopoulou, N.C. Karayiannis, M. Laso, M. Kröger, Structure, Dimensions, and Entanglement Statistics of Long Linear Polyethylene Chains, The Journal of Physical Chemistry B 113(2) (2009) 442-455.

[65] G. Tsolou, N. Stratikis, C. Baig, P.S. Stephanou, V.G. Mavrantzas, Melt Structure and Dynamics of Unentangled Polyethylene Rings: Rouse Theory, Atomistic Molecular Dynamics Simulation, and Comparison with the Linear Analogues, Macromolecules 43(24) (2010) 1069210713.

[66] L.D. Peristeras, I.G. Economou, D.N. Theodorou, Structure and Volumetric Properties of Linear and Triarm Star Polyethylenes from Atomistic Monte Carlo Simulation Using New Internal Rearrangement Moves, Macromolecules 38(2) (2005) 386-397.

[67] C. Baig, O. Alexiadis, V.G. Mavrantzas, Advanced Monte Carlo Algorithm for the Atomistic Simulation of Short- and Long-Chain Branched Polymers: Implementation for Model H-Shaped, A3AA3 Multiarm (Pom-Pom), and Short-Chain Branched Polyethylene Melts, Macromolecules 43(2) (2010) 986-1002.

[68] S.H. Jeong, J.M. Kim, J. Yoon, C. Tzoumanekas, M. Kroger, C. Baig, Influence of molecular architecture on the entanglement network: topological analysis of linear, long- and short-chain branched polyethylene melts via Monte Carlo simulations, Soft Matter 12(16) (2016) 37703786.

[69] J. Ramos, L.D. Peristeras, D.N. Theodorou, Monte Carlo Simulation of Short Chain Branched Polyolefins in the Molten State, Macromolecules 40(26) (2007) 9640-9650.

[70] J. Ramos, J.F. Vega, D.N. Theodorou, J. Martinez-Salazar, Entanglement Relaxation Time in Polyethylene: Simulation versus Experimental Data, Macromolecules 41(8) (2008) 2959-2962. [71] K. Moorthi, K. Kamio, J. Ramos, D.N. Theodorou, Monte Carlo Simulation of Short Chain Branched Polyolefins: Structure and Properties, Macromolecules 45(20) (2012) 8453-8466. [72] J. Ramos, J.F. Vega, J. Martinez-Salazar, Assessment of entanglement features and dynamics from atomistic simulations and experiments in linear and short chain branched polyolefins, Soft Matter 8(23) (2012) 6256-6263.

[73] J. Ramos, J.F. Vega, J. Martínez-Salazar, Molecular Dynamics Simulations for the Description of Experimental Molecular Conformation, Melt Dynamics, and Phase Transitions in Polyethylene, Macromolecules 48(14) (2015) 5016-5027.

[74] J. Ramos, J.F. Vega, J. Martinez-Salazar, A new insight into the conformation and melt dynamics of hydrogenated polybutadiene as revealed by computer simulations, Soft Matter 12(17) (2016) 3929-3936.

[75] N.C. Karayiannis, A.E. Giannousaki, V.G. Mavrantzas, An advanced Monte Carlo method for the equilibration of model long-chain branched polymers with a well-defined molecular architecture: Detailed atomistic simulation of an $\mathrm{H}$-shaped polyethylene melt, The Journal of Chemical Physics 118(6) (2003) 2451-2454.

[76] N.C. Karayiannis, V.G. Mavrantzas, Hierarchical Modeling of the Dynamics of Polymers with a Nonlinear Molecular Architecture: Calculation of Branch Point Friction and Chain Reptation Time of H-Shaped Polyethylene Melts from Long Molecular Dynamics Simulations, Macromolecules 38(20) (2005) 8583-8596. 
[77] P. Gestoso, N.C. Karayiannis, Molecular Simulation of the Effect of Temperature and Architecture on Polyethylene Barrier Properties, The Journal of Physical Chemistry B 112(18) (2008) 5646-5660.

[78] M.P. Allen, D.J. Tildesley, Computer Simulation of Liquids Clarendon Press1987.

[79] D.J. Lohse, The Influence of Chemical Structure on Polyolefin Melt Rheology and Miscibility, Journal of Macromolecular Science, Part C 45(4) (2005) 289-308.

[80] J. Han, R.L. Jaffe, D.Y. Yoon, Conformational Characteristics of Polymethylene Chains in Melts and in Various Phantom Chains from Explicit Atom Molecular Dynamics Simulations, Macromolecules 30(23) (1997) 7245-7252.

[81] M. Mondello, G.S. Grest, E.B. Webb, P. Peczak, Dynamics of n-alkanes: Comparison to Rouse model, The Journal of Chemical Physics 109(2) (1998) 798-805.

[82] A. Uhlherr, M. Doxastakis, V.G. Mavrantzas, D.N. Theodorou, S.J. Leak, N.E. Adam, P.E. Nyberg, Atomic structure of a high polymer melt, EPL (Europhysics Letters) 57(4) (2002) 506. [83] A. Uhlherr, S.J. Leak, N.E. Adam, P.E. Nyberg, M. Doxastakis, V.G. Mavrantzas, D.N. Theodorou, Large scale atomistic polymer simulations using Monte Carlo methods for parallel vector processors, Computer Physics Communications 144(1) (2002) 1-22.

[84] V.A. Harmandaris, V.G. Mavrantzas, D.N. Theodorou, M. Kröger, J. Ramírez, H.C. Öttinger, D. Vlassopoulos, Crossover from the Rouse to the Entangled Polymer Melt Regime: Signals from Long, Detailed Atomistic Molecular Dynamics Simulations, Supported by Rheological Experiments, Macromolecules 36(4) (2003) 1376-1387.

[85] B.J. Banaszak, J.J. de Pablo, A new double-rebridging technique for linear polyethylene, The Journal of Chemical Physics 119(4) (2003) 2456-2462.

[86] P. Carbone, H. Ali Karimi-Varzaneh, F. Muller-Plathe, Fine-graining without coarsegraining: an easy and fast way to equilibrate dense polymer melts, Faraday Discussions 144(0) (2010) 25-42.

[87] J.A. Martins, N.M. Micaelo, Short-Range Order in Polyethylene Melts: Identification and Characterization, Macromolecules 46(19) (2013) 7977-7988.

[88] P.-N. Tzounis, S.D. Anogiannakis, D.N. Theodorou, General Methodology for Estimating the Stiffness of Polymer Chains from Their Chemical Constitution: A Single Unperturbed Chain Monte Carlo Algorithm, Macromolecules 50(11) (2017) 4575-4587.

[89] J.T. Padding, W.J. Briels, Uncrossability constraints in mesoscopic polymer melt simulations: Non-Rouse behavior of $\mathrm{C} 12 \mathrm{OH} 242$, The Journal of Chemical Physics 115(6) (2001) 2846-2859.

[90] J.T. Padding, W.J. Briels, Time and length scales of polymer melts studied by coarsegrained molecular dynamics simulations, The Journal of Chemical Physics 117(2) (2002) 925943.

[91] H.S. Ashbaugh, H.A. Patel, S.K. Kumar, S. Garde, Mesoscale model of polymer melt structure: Self-consistent mapping of molecular correlations to coarse-grained potentials, The Journal of Chemical Physics 122(10) (2005) 104908.

[92] P.K. Depa, J.K. Maranas, Dynamic evolution in coarse-grained molecular dynamics simulations of polyethylene melts, The Journal of Chemical Physics 126(5) (2007) 054903. [93] P.K. Depa, J.K. Maranas, Speed up of dynamic observables in coarse-grained moleculardynamics simulations of unentangled polymers, The Journal of Chemical Physics 123(9) (2005) 094901.

[94] K.M. Salerno, A. Agrawal, D. Perahia, G.S. Grest, Resolving Dynamic Properties of Polymers through Coarse-Grained Computational Studies, Physical Review Letters 116(5) (2016) 058302.

[95] K.M. Salerno, A. Agrawal, B.L. Peters, D. Perahia, G.S. Grest, Dynamics in entangled polyethylene melts, The European Physical Journal Special Topics 225(8) (2016) 1707-1722.

[96] B.L. Peters, K.M. Salerno, A. Agrawal, D. Perahia, G.S. Grest, Coarse-Grained Modeling of Polyethylene Melts: Effect on Dynamics, Journal of Chemical Theory and Computation 13(6) (2017) 2890-2896. 
[97] A.P. Sgouros, G. Megariotis, D.N. Theodorou, Slip-Spring Model for the Linear and Nonlinear Viscoelastic Properties of Molten Polyethylene Derived from Atomistic Simulations, Macromolecules 50(11) (2017) 4524-4541.

[98] C. Jeong, J.F. Douglas, Mass dependence of the activation enthalpy and entropy of unentangled linear alkane chains, The Journal of Chemical Physics 143(14) (2015) 144905. [99] J. Baschnagel, K. Qin, W. Paul, K. Binder, Monte Carlo simulation of models for single polyethylene coils, Macromolecules 25(12) (1992) 3117-3124.

[100] D. Heine, D.T. Wu, J.G. Curro, G.S. Grest, Role of intramolecular energy on polyolefin miscibility: Isotactic polypropylene/polyethylene blends, The Journal of Chemical Physics 118(2) (2002) 914-924.

[101] R. Chiang, Intrinsic Viscosity-Molecular Weight Relationship for Fractions of Linear Polyethylene1, The Journal of Physical Chemistry 69(5) (1965) 1645-1653.

[102] R. Chiang, Temperature Coefficient of the Unperturbed Dimension of Linear Polyethylene from Intrinsic Viscosity Measurements in $\theta$ Solvents, The Journal of Physical Chemistry 70(7) (1966) 2348-2352.

[103] A. Nakajima, H. Fujiwara, F. Hamada, Phase relationships and thermodynamic interactions in linear polyethylene-diluent systems, Journal of Polymer Science Part A-2: Polymer Physics 4(3) (1966) 507-518.

[104] G. Lieser, E.W. Fischer, K. Ibel, Conformation of polyethylene molecules in the melt as revealed by small-angle neutron scattering, Journal of Polymer Science: Polymer Letters Edition 13(1) (1975) 39-43.

[105] J. Schelten, D.G.H. Ballard, G.D. Wignall, G. Longman, W. Schmatz, Small-angle neutron scattering studies of molten and crystalline polyethylene, Polymer 17(9) (1976) 751-757.

[106] Y. Muraoka, K. Kamide, H. Suzuki, Direct determinations of the unperturbed dimensions of linear polyethylene molecules, British Polymer Journal 15(2) (1983) 107-109.

[107] B. Crist, J.D. Tanzer, W.W. Graessley, Small-angle neutron scattering of a model crystallizable polymer: Crystallization and size-mismatch effects, Journal of Polymer Science Part B: Polymer Physics 25(3) (1987) 545-556.

[108] J.C. Horton, G.L. Squires, A.T. Boothroyd, L.J. Fetters, A.R. Rennie, C.J. Glinka, R.A. Robinson, Small-angle neutron scattering from star-branched polymers in the molten state, Macromolecules 22(2) (1989) 681-686.

[109] A.T. Bootheroyd, A.R. Rennie, C.B. Boothroyd, Direct Measurement of the Temperature Dependence of the Unperturbed Dimensions of a Polymer, EPL (Europhysics Letters) 15(7) (1991) 715.

[110] M. Zamponi, A. Wischnewski, M. Monkenbusch, L. Willner, D. Richter, P. Falus, B. Farago, M.G. Guenza, Cooperative Dynamics in Homopolymer Melts: A Comparison of Theoretical Predictions with Neutron Spin Echo Experiments, The Journal of Physical Chemistry B 112(50) (2008) 16220-16229.

[111] T. Sun, P. Brant, R.R. Chance, W.W. Graessley, Effect of Short Chain Branching on the Coil Dimensions of Polyolefins in Dilute Solution, Macromolecules 34(19) (2001) 6812-6820.

[112] A. Neelakantan, R. Stine, J.K. Maranas, Chain Packing in Ethylene-Butene Copolymers, Macromolecules 36(10) (2003) 3721-3731.

[113] E. Panizon, D. Bochicchio, L. Monticelli, G. Rossi, MARTINI Coarse-Grained Models of Polyethylene and Polypropylene, The Journal of Physical Chemistry B 119(25) (2015) 82098216.

[114] H. Li, J.G. Curro, D.T. Wu, A. Habenschuss, X-ray Scattering of Vinyl Polyolefin Liquids and Random Copolymers: Theory and Experiment, Macromolecules 41(7) (2008) 2694-2700.

[115] N. Hadjichristidis, M. Xenidou, H. latrou, M. Pitsikalis, Y. Poulos, A. Avgeropoulos, S. Sioula, S. Paraskeva, G. Velis, D.J. Lohse, D.N. Schulz, L.J. Fetters, P.J. Wright, R.A. Mendelson, C.A. García-Franco, T. Sun, C.J. Ruff, Well-Defined, Model Long Chain Branched Polyethylene. 1. Synthesis and Characterization, Macromolecules 33(7) (2000) 2424-2436. 
[116] C.J. Stacy, R.L. Arnett, Intrinsic Viscosity of Linear Polyethylene in a $\theta$-Solvent, The Journal of Physical Chemistry 69(9) (1965) 3109-3112.

[117] P.J. Flory, A. Ciferri, R. Chiang, Temperature Coefficient of the Polyethylene Chain Conformation from Intrinsic Viscosity Measurements1, Journal of the American Chemical Society 83(5) (1961) 1023-1026.

[118] A. Ciferri, C.A.J. Hoeve, P.J. Flory, Stress-Temperature Coefficients of Polymer Networks and the Conformational Energy of Polymer Chains1, Journal of the American Chemical Society 83(5) (1961) 1015-1022.

[119] C. Price, F.-P. Wolf, Thermodynamics of Rubber Elasticity [and Discussion], Proceedings of the Royal Society of London. A. Mathematical and Physical Sciences 351(1666) (1976) 331350.

[120] B.J. Banaszak, R. Faller, J.J. de Pablo, Simulation of the effects of chain architecture on the sorption of ethylene in polyethylene, The Journal of Chemical Physics 120(23) (2004) 11304-11315.

[121] F.J.B. Calleja, J.C.G. Ortega, J.M. de Salazar, Distribution of chain defects and microstructure of melt crystallized polyethylene, Polymer 19(9) (1978) 1094-1099.

[122] J. Martinez De Salazar, F.J. BaltáCalleja, Influence of chain defects on the crystallization of polyethylene with reference to crystal size and perfection, Journal of Crystal Growth 48(2) (1980) 283-294.

[123] J.M. Salazar, F.J.B. Calleja, On the inclusion of chain defects in the polyethylene lattice a statistical approach, Polymer Bulletin 2(3) (1980) 163-167.

[124] S. Martín, J.F. Vega, M.T. Expósito, A. Flores, J. Martínez-Salazar, A three-phase microstructural model to explain the mechanical relaxations of branched polyethylene: a DSC, WAXD and DMTA combined study, Colloid and Polymer Science 289(3) (2011) 257-268.

[125] M. Doi, S.F. Edwards, The Theory of Polymer Dynamics, Clarendon Press 1988.

[126] P.G. de Gennes, Scaling Concepts in Polymer Physics, Cornell University Press1979.

[127] W.W. Graessley, S.F. Edwards, Entanglement interactions in polymers and the chain contour concentration, Polymer 22(10) (1981) 1329-1334.

[128] L.J. Fetters, D.J. Lohse, D. Richter, T.A. Witten, A. Zirkel, Connection between Polymer Molecular Weight, Density, Chain Dimensions, and Melt Viscoelastic Properties,

Macromolecules 27(17) (1994) 4639-4647.

[129] L.J. Fetters, D.J. Lohse, W.W. Graessley, Chain dimensions and entanglement spacings in dense macromolecular systems, Journal of Polymer Science Part B: Polymer Physics 37(10)

(1999) 1023-1033.

[130] L.J. Fetters, D.J. Lohse, S.T. Milner, W.W. Graessley, Packing Length Influence in Linear

Polymer Melts on the Entanglement, Critical, and Reptation Molecular Weights,

Macromolecules 32(20) (1999) 6847-6851.

[131] L.J. Fetters, D.J. Lohse, C.A. García-Franco, P. Brant, D. Richter, Prediction of Melt State Poly( $\alpha$-olefin) Rheological Properties: The Unsuspected Role of the Average Molecular Weight per Backbone Bond, Macromolecules 35(27) (2002) 10096-10101.

[132] J.F. Vega, J. Ramos, J. Martínez-Salazar, Effect of short chain branching in molecular dimensions and Newtonian viscosity of ethylene/1-hexene copolymers: matching conformational and rheological experimental properties and atomistic simulations, Rheologica Acta 53(1) (2014) 1-13.

[133] J.F. Vega, S. Martín, M.T. Expósito, J. Martínez-Salazar, Entanglement network and relaxation temperature dependence of single-site catalyzed ethylene/1-hexene copolymers, Journal of Applied Polymer Science 109(3) (2008) 1564-1569.

[134] C.A. García-Franco, B.A. Harrington, D.J. Lohse, Effect of Short-Chain Branching on the Rheology of Polyolefins, Macromolecules 39(7) (2006) 2710-2717.

[135] C.A. García-Franco, B.A. Harrington, D.J. Lohse, On the rheology of ethylene-octene copolymers, Rheologica Acta 44(6) (2005) 591-599. 
[136] X. Chen, F.J. Stadler, H. Münstedt, R.G. Larson, Method for obtaining tube model parameters for commercial ethene/ $\alpha$-olefin copolymers, Journal of Rheology 54(2) (2010) 393406.

[137] A.E. Likhtman, T.C.B. McLeish, Quantitative Theory for Linear Dynamics of Linear Entangled Polymers, Macromolecules 35(16) (2002) 6332-6343.

[138] R. Everaers, S.K. Sukumaran, G.S. Grest, C. Svaneborg, A. Sivasubramanian, K. Kremer, Rheology and Microscopic Topology of Entangled Polymeric Liquids, Science 303(5659) (2004) 823-826.

[139] Q. Zhou, R.G. Larson, Primitive Path Identification and Statistics in Molecular Dynamics Simulations of Entangled Polymer Melts, Macromolecules 38(13) (2005) 5761-5765.

[140] M. Kröger, Shortest multiple disconnected path for the analysis of entanglements in twoand three-dimensional polymeric systems, Computer Physics Communications 168(3) (2005) 209-232.

[141] C. Tzoumanekas, D.N. Theodorou, Topological Analysis of Linear Polymer Melts: A Statistical Approach, Macromolecules 39(13) (2006) 4592-4604.

[142] P.S. Stephanou, C. Baig, G. Tsolou, V.G. Mavrantzas, M. Kröger, Quantifying chain reptation in entangled polymer melts: Topological and dynamical mapping of atomistic simulation results onto the tube model, The Journal of Chemical Physics 132(12) (2010) 124904.

[143] P.S. Stephanou, C. Baig, V.G. Mavrantzas, Projection of atomistic simulation data for the dynamics of entangled polymers onto the tube theory: calculation of the segment survival probability function and comparison with modern tube models, Soft Matter 7(2) (2011) 380 395.

[144] P.S. Stephanou, V.G. Mavrantzas, Quantitative predictions of the linear viscoelastic properties of entangled polyethylene and polybutadiene melts via modified versions of modern tube models on the basis of atomistic simulation data, Journal of Non-Newtonian Fluid Mechanics 200(Supplement C) (2013) 111-130.

[145] D.J. Read, K. Jagannathan, A.E. Likhtman, Entangled Polymers: Constraint Release, Mean Paths, and Tube Bending Energy, Macromolecules 41(18) (2008) 6843-6853.

[146] W. Bisbee, J. Qin, S.T. Milner, Finding the Tube with Isoconfigurational Averaging, Macromolecules 44(22) (2011) 8972-8980.

[147] J. Qin, S.T. Milner, Tubes, Topology, and Polymer Entanglement, Macromolecules 47(17) (2014) 6077-6085.

[148] J. Cao, J. Qin, S.T. Milner, Simulating Constraint Release by Watching a Ring Cross Itself, Macromolecules 47(7) (2014) 2479-2486.

[149] K. Kremer, G.S. Grest, Dynamics of entangled linear polymer melts: A moleculardynamics simulation, The Journal of Chemical Physics 92(8) (1990) 5057-5086.

[150] M. Pütz, K. Kremer, G.S. Grest, What is the entanglement length in a polymer melt?, Europhys. Lett. 49(6) (2000) 735-741.

[151] T. Kreer, J. Baschnagel, M. Müller, K. Binder, Monte Carlo Simulation of Long Chain Polymer Melts: Crossover from Rouse to Reptation Dynamics, Macromolecules 34(4) (2001) 1105-1117.

[152] J.-X. Hou, Note: Determine entanglement length through monomer mean-square displacement, The Journal of Chemical Physics 146(2) (2017) 026101.

[153] M.H. Nafar Sefiddashti, B.J. Edwards, B. Khomami, Steady shearing flow of a moderately entangled polyethylene liquid, Journal of Rheology 60(6) (2016) 1227-1244.

[154] J. van Meerveld, A method to extract the monomer friction coefficient from the linear viscoelastic behavior of linear, entangled polymer melts, Rheologica Acta 43(6) (2004) 615623.

[155] J.F. Vega, S. Rastogi, G.W.M. Peters, H.E.H. Meijer, Rheology and reptation of linear polymers. Ultrahigh molecular weight chain dynamics in the melt, Journal of Rheology 48(3) (2004) 663-678. 
[156] P. Schleger, B. Farago, C. Lartigue, A. Kollmar, D. Richter, Clear Evidence of Reptation in Polyethylene from Neutron Spin-Echo Spectroscopy, Physical Review Letters 81(1) (1998) 124127.

[157] A. Wischnewski, M. Monkenbusch, L. Willner, D. Richter, A.E. Likhtman, T.C.B. McLeish, B. Farago, Molecular Observation of Contour-Length Fluctuations Limiting Topological Confinement in Polymer Melts, Physical Review Letters 88(5) (2002) 058301.

[158] L. Szántó, R. Vogt, J. Meier, D. Auhl, E. Van Ruymbeke, C. Friedrich, Entanglement relaxation time of polyethylene melts from high-frequency rheometry in the mega-hertz range, Journal of Rheology 61(5) (2017) 1023-1033.

[159] J.T. Padding, W.J. Briels, A time-integrated estimate of the entanglement mass in polymer melts in agreement with the one determined by time-resolved measurements, The Journal of Chemical Physics 120(6) (2004) 2996-3002.

[160] A. Wischnewski, M. Monkenbusch, L. Willner, D. Richter, G. Kali, Direct Observation of the Transition from Free to Constrained Single-Segment Motion in Entangled Polymer Melts, Physical Review Letters 90(5) (2003) 058302.

[161] D. Richter, B. Farago, R. Butera, L.J. Fetters, J.S. Huang, B. Ewen, On the origins of entanglement constraints, Macromolecules 26(4) (1993) 795-804.

[162] F. Bueche, Viscosity, Self-Diffusion, and Allied Effects in Solid Polymers, The Journal of Chemical Physics 20(12) (1952) 1959-1964.

[163] J. Luettmer-Strathmann, Effect of small-scale architecture on polymer mobility, The Journal of Chemical Physics 112(12) (2000) 5473-5479.

[164] G.D. Smith, W. Paul, D.Y. Yoon, A. Zirkel, J. Hendricks, D. Richter, H. Schober, Local dynamics in a long-chain alkane melt from molecular dynamics simulations and neutron scattering experiments, The Journal of Chemical Physics 107(12) (1997) 4751-4755.

[165] M. Guenza, Intermolecular Effects in the Center-of-Mass Dynamics of Unentangled Polymer Fluids, Macromolecules 35(7) (2002) 2714-2722.

[166] M. Guenza, Cooperative Dynamics in Unentangled Polymer Fluids, Physical Review Letters 88(2) (2001) 025901.

[167] M. Guenza, Many chain correlated dynamics in polymer fluids, The Journal of Chemical Physics 110(15) (1999) 7574-7588.

[168] N. Di Pasquale, P. Carbone, Local and global dynamics of multi-resolved polymer chains: Effects of the interactions atoms-beads on the dynamic of the chains, The Journal of Chemical Physics 146(8) (2017) 084905.

[169] T.C.B. McLeish, R.G. Larson, Molecular constitutive equations for a class of branched polymers: The pom-pom polymer, Journal of Rheology 42(1) (1998) 81-110.

[170] E. Lee, S. Kim, Y. Jung, Slowing Down of Ring Polymer Diffusion Caused by Inter-Ring Threading, Macromolecular Rapid Communications 36(11) (2015) 1115-1121.

[171] V.A. Harmandaris, V.G. Mavrantzas, D.N. Theodorou, Atomistic Molecular Dynamics Simulation of Stress Relaxation upon Cessation of Steady-State Uniaxial Elongational Flow, Macromolecules 33(21) (2000) 8062-8076.

[172] J. Vega, M. Aguilar, J. Peón, D. Pastor, J. Martínez-Salazar, Effect of long chain branching on linear-viscoelastic melt properties of polyolefins, e-Polymers 2(1) (2002).

[173] J.F. Vega, J. Otegui, J. Ramos, J. Martínez-Salazar, Effect of molecular weight distribution on Newtonian viscosity of linear polyethylene, Rheologica Acta 51(1) (2012) 81-87.

[174] J. Otegui, J. Ramos, J.F. Vega, J. Martínez-Salazar, Effect of high molar mass species on linear viscoelastic properties of polyethylene melts, European Polymer Journal 49(9) (2013) 2748-2758.

[175] A.E. Likhtman, S.K. Sukumaran, J. Ramirez, Linear Viscoelasticity from Molecular Dynamics Simulation of Entangled Polymers, Macromolecules 40(18) (2007) 6748-6757.

[176] K. Takahashi, R. Nishimura, K. Yasuoka, Y. Masubuchi, Molecular Dynamics Simulations for Resolving Scaling Laws of Polyethylene Melts, Polymers 9(1) (2017) 24. 
[177] J.D. Moore, S.T. Cui, H.D. Cochran, P.T. Cummings, A molecular dynamics study of a short-chain polyethylene melt.: I. Steady-state shear, Journal of Non-Newtonian Fluid Mechanics 93(1) (2000) 83-99.

[178] J.D. Moore, S.T. Cui, H.D. Cochran, P.T. Cummings, A molecular dynamics study of a short-chain polyethylene melt.: II. Transient response upon onset of shear, Journal of NonNewtonian Fluid Mechanics 93(1) (2000) 101-116.

[179] C. Baig, B.J. Edwards, D.J. Keffer, H.D. Cochran, Rheological and structural studies of liquid decane, hexadecane, and tetracosane under planar elongational flow using nonequilibrium molecular-dynamics simulations, The Journal of Chemical Physics 122(18) (2005) 184906.

[180] C. Baig, B.J. Edwards, D.J. Keffer, H.D. Cochran, A proper approach for nonequilibrium molecular dynamics simulations of planar elongational flow, The Journal of Chemical Physics 122(11) (2005) 114103.

[181] C. Baig, B.J. Edwards, D.J. Keffer, H.D. Cochran, V.A. Harmandaris, Rheological and structural studies of linear polyethylene melts under planar elongational flow using nonequilibrium molecular dynamics simulations, The Journal of Chemical Physics 124(8) (2006) 084902.

[182] C. Baig, B.J. Edwards, D.J. Keffer, A molecular dynamics study of the stress-optical behavior of a linear short-chain polyethylene melt under shear, Rheologica Acta 46(9) (2007) 1171-1186.

[183] C. Baig, V.G. Mavrantzas, M. Kröger, Flow Effects on Melt Structure and Entanglement Network of Linear Polymers: Results from a Nonequilibrium Molecular Dynamics Simulation Study of a Polyethylene Melt in Steady Shear, Macromolecules 43(16) (2010) 6886-6902. [184] S. Jeong, J.M. Kim, C. Baig, Effect of Chain Orientation and Stretch on the Stress Overshoot of Entangled Polymeric Materials under Start-Up Shear, Macromolecules 50(8) (2017) 3424-3429.

[185] C. Baig, V.G. Mavrantzas, Tension thickening, molecular shape, and flow birefringence of an $\mathrm{H}$-shaped polymer melt in steady shear and planar extension, The Journal of Chemical Physics 132(1) (2010) 014904.

[186] J.M. Kim, C. Baig, Precise Analysis of Polymer Rotational Dynamics, 6 (2016) 19127. [187] J.M. Kim, C. Baig, Communication: Role of short chain branching in polymer structure and dynamics, The Journal of Chemical Physics 144(8) (2016) 081101.

[188] S.H. Jeong, J.M. Kim, C. Baig, Rheological Influence of Short-Chain Branching for Polymeric Materials under Shear with Variable Branch Density and Branching Architecture, Macromolecules 50(11) (2017) 4491-4500.

[189] S. Cho, S. Jeong, J.M. Kim, C. Baig, Molecular dynamics for linear polymer melts in bulk and confined systems under shear flow, Scientific Reports 7(1) (2017) 9004.

[190] S. Jeong, S. Cho, J.M. Kim, C. Baig, Molecular mechanisms of interfacial slip for polymer melts under shear flow, Journal of Rheology 61(2) (2017) 253-264.

[191] M.H. Nafar Sefiddashti, B.J. Edwards, B. Khomami, Evaluation of reptation-based modeling of entangled polymeric fluids including chain rotation via nonequilibrium molecular dynamics simulation, Physical Review Fluids 2(8) (2017) 083301.

[192] J.T. Padding, W.J. Briels, Coarse-grained molecular dynamics simulations of polymer melts in transient and steady shear flow, The Journal of Chemical Physics 118(22) (2003) 10276-10286.

[193] M. Aguilar, M.T. Expósito, J.F. Vega, A. Muñoz-Escalona, J. Martínez-Salazar, Elimination of Extrudate Distortions in Metallocene-Catalyzed Polyethylene, Macromolecules 37(3) (2004) 681-683.

[194] M. Aguilar, S. Martín, J.F. Vega, A. Muñoz-Escalona, J. Martínez-Salazar, Processability of a metallocene-catalyzed linear PE improved by blending with a small amount of UHMWPE, Journal of Polymer Science Part B: Polymer Physics 43(21) (2005) 2963-2971. 
[195] J.F. Vega, M.T. Expósito, J. Otegui, J. Martínez-Salazar, Eliminating sharkskin distortion in polyethylene extrusion via a molecular route, Journal of Rheology 55(4) (2011) 855-873.

[196] S.G. Hatzikiriakos, Wall slip of molten polymers, Progress in Polymer Science 37(4) (2012) 624-643.

[197] M. Aguilar, J.F. Vega, E. Sanz, J. Martínez-Salazar, New aspects on the rheological behaviour of metallocene catalysed polyethylenes, Polymer 42(24) (2001) 9713-9721. [198] M. Aguilar, J.F. Vega, A. Muñoz-Escalona, J. Martínez-Salazar, Temperature and branching dependence of surface extrusion instabilities in metallocene catalysed polyethylene, Journal of Materials Science 37(16) (2002) 3415-3421.

[199] R. Popli, M. Glotin, L. Mandelkern, R.S. Benson, Dynamic mechanical studies of $\alpha$ and $\beta$ relaxations of polyethylenes, Journal of Polymer Science: Polymer Physics Edition 22(3) (1984) 407-448.

[200] R.H. Boyd, Strengths of the mechanical $\alpha, \beta$ - and $\psi$-relaxation processes in linear polyethylene, Macromolecules 17(4) (1984) 903-911.

[201] R.H. Boyd, Relaxation processes in crystalline polymers: experimental behaviour - a review, Polymer 26(3) (1985) 323-347.

[202] R.H. Boyd, Relaxation processes in crystalline polymers: molecular interpretation - a review, Polymer 26(8) (1985) 1123-1133.

[203] V.E.W. Fischer, A. Peterlin, Kernresonanzmessungen zur untersuchung der kettenbeweglichkeit in polyäthylen-einkristallen, Die Makromolekulare Chemie 74(1) (1964) 128.

[204] K.-H. Illers, Innere Rotationen in Festkörpern aus hoch- und niedrigmolekularen organischen Molekülen, Rheologica Acta 3(4) (1964) 194-202.

[205] J.A. Sayre, S.R. Swanson, R.H. Boyd, The effect of pressure on the volume and the dielectric relaxation of linear polyethylene, Journal of Polymer Science: Polymer Physics Edition 16(10) (1978) 1739-1759.

[206] D.E. Kline, J.A. Sauer, A.E. Woodward, Effect of branching on dynamic mechanical properties of polyethylene, Journal of Polymer Science 22(102) (1956) 455-462.

[207] J.M. Crissman, On the transition from paraffinic to polymeric behavior: Mechanical properties, Journal of Polymer Science: Polymer Physics Edition 13(7) (1975) 1407-1416.

[208] A.H. Willbourn, The glass transition in polymers with the $(\mathrm{CH} 2)$ group, Transactions of the Faraday Society 54(0) (1958) 717-729.

[209] T.F. Schatzki, Statistical computation of distribution functions of dimensions of macromolecules, Journal of Polymer Science 57(165) (1962) 337-356.

[210] R.F. Boyer, The Relation of Transition Temperatures to Chemical Structure in High Polymers, Rubber Chemistry and Technology 36(5) (1963) 1303-1421.

[211] R.C. Rempel, H.E. Weaver, R.H. Sands, R.L. Miller, Nuclear Magnetic Resonance Studies of Polyethylene, Journal of Applied Physics 28(10) (1957) 1082-1089.

[212] G.P. Mikhailov, S.P. Kabin, T.A. Krylova, Dielectric and mechanical losses of low-pressure polyethylene Soviet Phys.-Tech. Phys 2 (1957) 1899-1903.

[213] N.G. McCrum, B.E. Read, G. Williams, Anelastic and dïelectric effects in polymeric solids, John Wiley1967.

[214] Z.H. Stachurski, I.M. Ward, Mechanical relaxations in polyethylene, Journal of Macromolecular Science, Part B 3(3) (1969) 445-494.

[215] R.S. Stein, Rheo-optical and dielectric studies of the $\alpha$ loss region of low-density polyethylene, Journal of Macromolecular Science, Part B 8(1-2) (1973) 29-40.

[216] T. Kajiyama, T. Okada, A. Sakoda, M. Takayanagi, Analysis of the $\alpha$-relaxation process of bulk crystallized polyethylene based on that of single crystal mat, Journal of Macromolecular Science, Part B 7(3) (1973) 583-608.

[217] A. Danch, W. Osoba, F. Stelzer, On the $\alpha$ relaxation of the constrained amorphous phase in poly(ethylene), European Polymer Journal 39(10) (2003) 2051-2058. 
[218] R.F. Boyer, An apparent double glass transition in semicrystalline polymers, Journal of Macromolecular Science, Part B 8(3-4) (1973) 503-537.

[219] H. Sha, X. Zhang, I.R. Harrison, A dynamic mechanical thermal analysis (DMTA) study of polyethylenes, Thermochimica Acta 192(Supplement C) (1991) 233-242.

[220] K. Bergmann, K. Nawotki, Eine neue Interpretation der Breitlinien-Kernresonanzspektren von linearem Polyäthylen, Kolloid-Zeitschrift und Zeitschrift für Polymere 219(2) (1967) 132-

144.

[221] R. Kitamaru, F. Horii, S.H. Hyon, Proton magnetic resonance studies of the phase structure of bulk-crystallized linear polyethylene, Journal of Polymer Science: Polymer Physics Edition 15(5) (1977) 821-836.

[222] G.R. Strobl, W. Hagedorn, Raman spectroscopic method for determining the crystallinity of polyethylene, Journal of Polymer Science: Polymer Physics Edition 16(7) (1978) 1181-1193.

[223] P. Sajkiewicz, T. Hashimoto, K. Saijo, A. Gradys, 'Intermediate phase' in poly(ethylene) as elucidated by the WAXS. Analysis of crystallization kinetics, Polymer 46(2) (2005) 513-521.

[224] R.F. Boyer, Glass Temperatures of Polyethylene, Macromolecules 6(2) (1973) 288-299.

[225] S.Z.D. Cheng, B. Wunderlich, Glass transition and melting behavior of poly(ethylene 2,6naphthalenedicarboxylate), Macromolecules 21(3) (1988) 789-797.

[226] A. Sedighiamiri, T.B. Van Erp, G.W.M. Peters, L.E. Govaert, J.A.W. van Dommelen, Micromechanical modeling of the elastic properties of semicrystalline polymers: A three-phase approach, Journal of Polymer Science Part B: Polymer Physics 48(20) (2010) 2173-2184.

[227] R. Simha, R.F. Boyer, On a General Relation Involving the Glass Temperature and Coefficients of Expansion of Polymers, The Journal of Chemical Physics 37(5) (1962) 1003-1007. [228] R. Brüning, K. Samwer, Glass transition on long time scales, Physical Review B 46(18) (1992) 11318-11322.

[229] J. Buchholz, W. Paul, F. Varnik, K. Binder, Cooling rate dependence of the glass transition temperature of polymer melts: Molecular dynamics study, The Journal of Chemical Physics 117(15) (2002) 7364-7372.

[230] K. Binder, J. Baschnagel, W. Paul, Glass transition of polymer melts: test of theoretical concepts by computer simulation, Progress in Polymer Science 28(1) (2003) 115-172.

[231] A.V. Lyulin, N.K. Balabaev, M.A.J. Michels, Molecular-Weight and Cooling-Rate Dependence of Simulated Tg for Amorphous Polystyrene, Macromolecules 36(22) (2003) 85748575.

[232] P. Wolfgang, D.S. Grant, Structure and dynamics of amorphous polymers: computer simulations compared to experiment and theory, Reports on Progress in Physics 67(7) (2004) 1117.

[233] P. Yi, C.R. Locker, G.C. Rutledge, Molecular Dynamics Simulation of Homogeneous Crystal Nucleation in Polyethylene, Macromolecules 46(11) (2013) 4723-4733.

[234] R. Wu, X. Qiu, T. Zhang, K. Fu, X. Yang, Atomistic Molecular Insight into the Time Dependence of Polymer Glass Transition, The Journal of Physical Chemistry B 119(30) (2015) 9959-9969.

[235] Q. Yang, X. Chen, Z. He, F. Lan, H. Liu, The glass transition temperature measurements of polyethylene: determined by using molecular dynamic method, RSC Advances 6(15) (2016) 12053-12060.

[236] E.B. Stukalin, J.F. Douglas, K.F. Freed, Application of the entropy theory of glass formation to poly( $\alpha$-olefins), The Journal of Chemical Physics 131(11) (2009) 114905. [237] X. Luo, S. Xie, J. Liu, H. Hu, J. Jiang, W. Huang, H. Gao, D. Zhou, Z. Lu, D. Yan, The relationship between the degree of branching and glass transition temperature of branched polyethylene: experiment and simulation, Polymer Chemistry 5(4) (2014) 1305-1312. [238] A. Moyassari, M. Unge, M.S. Hedenqvist, U.W. Gedde, F. Nilsson, First-principle simulations of electronic structure in semicrystalline polyethylene, The Journal of Chemical Physics 146(20) (2017) 204901. 
[239] T.H. Pham, R. Ramprasad, H.-V. Nguyen, Density-functional description of polymer crystals: A comparative study of recent van der Waals functionals, The Journal of Chemical Physics 144(21) (2016) 214905.

[240] B. Montanari, P. Ballone, R.O. Jones, Density functional study of molecular crystals: Polyethylene and a crystalline analog of bisphenol-A polycarbonate, The Journal of Chemical Physics 108(16) (1998) 6947-6951.

[241] C.W. Bunn, T.C. Alcock, The texture of polythene, Transactions of the Faraday Society 41(0) (1945) 317-325.

[242] P.R. Swan, Polyethylene unit cell variations with branching, Journal of Polymer Science 56(164) (1962) 409-416.

[243] J. Otegui, J.F. Vega, S. Martín, V. Cruz, A. Flores, C. Domingo, J. Martínez-Salazar, The unit cell expansion of branched polyethylene as detected by Raman spectroscopy: an experimental and simulation approach, Journal of Materials Science 42(3) (2007) 1046-1049.

[244] J.F. Vega, N. Jargour, R. Núñez-Ramírez, G. Liu, D. Wang, M. Trujillo, A.J. Müller, J. Martínez-Salazar, The influence of short-chain branching on the morphology and structure of polyethylene single crystals, Journal of Polymer Science Part B: Polymer Physics 53(24) (2015) 1751-1762.

[245] G.D. Barrera, S.F. Parker, A.J. Ramirez-Cuesta, P.C.H. Mitchell, The Vibrational Spectrum and Ultimate Modulus of Polyethylene, Macromolecules 39(7) (2006) 2683-2690.

[246] S. Serra, S. Iarlori, E. Tosatti, S. Scandolo, G. Santoro, Dynamical and thermal properties of polyethylene by ab initio simulation, Chemical Physics Letters 331(2-4) (2000) 339-345.

[247] V. Barone, M. Casarin, D. Forrer, M. Pavone, M. Sambi, A. Vittadini, Role and effective treatment of dispersive forces in materials: Polyethylene and graphite crystals as test cases, Journal of Computational Chemistry 30(6) (2009) 934-939.

[248] J. Kleis, B.I. Lundqvist, D.C. Langreth, E. Schröder, Towards a working density-functional theory for polymers: First-principles determination of the polyethylene crystal structure, Physical Review B 76(10) (2007) 100201.

[249] C.-S. Liu, G. Pilania, C. Wang, R. Ramprasad, How Critical Are the van der Waals Interactions in Polymer Crystals?, The Journal of Physical Chemistry A 116(37) (2012) 93479352.

[250] M. Dion, H. Rydberg, E. Schröder, D.C. Langreth, B.I. Lundqvist, Van der Waals Density Functional for General Geometries, Physical Review Letters 92(24) (2004) 246401.

[251] G. Román-Pérez, J.M. Soler, Efficient Implementation of a van der Waals Density Functional: Application to Double-Wall Carbon Nanotubes, Physical Review Letters 103(9) (2009) 096102.

[252] J. Wu, F. Gygi, A simplified implementation of van der Waals density functionals for firstprinciples molecular dynamics applications, The Journal of Chemical Physics 136(22) (2012) 224107.

[253] R.A. Sorensen, W.B. Liau, L. Kesner, R.H. Boyd, Prediction of polymer crystal structures and properties: polyethylene and poly(oxymethylene), Macromolecules 21(1) (1988) 200-208. [254] I.-E. Mavrantza, D. Prentzas, V.G. Mavrantzas, C. Galiotis, Detailed atomistic moleculardynamics simulation of the orthorhombic phase of crystalline polyethylene and alkane crystals, The Journal of Chemical Physics 115(8) (2001) 3937-3950.

[255] T.L. Phillips, S. Hanna, A comparison of computer models for the simulation of crystalline polyethylene and the long n-alkanes, Polymer 46(24) (2005) 11003-11018.

[256] D.J. Lacks, G.C. Rutledge, Simulation of the temperature dependence of mechanical properties of polyethylene, The Journal of Physical Chemistry 98(4) (1994) 1222-1231.

[257] M.P. Howard, S.T. Milner, Self-Consistent Real Space Free Energy Calculations for Polyethylene and Isotactic Polypropylene Crystals and Interfaces, Macromolecules 47(23) (2014) 8335-8350.

[258] B. Crist, C.J. Fisher, P.R. Howard, Mechanical properties of model polyethylenes: tensile elastic modulus and yield stress, Macromolecules 22(4) (1989) 1709-1718. 
[259] J.F. Vega, A.J. Müller, J. Martínez-Salazar, New habits in branched polyethylene single crystals, European Polymer Journal 80(Supplement C) (2016) 169-174.

[260] P.R. Howard, B. Crist, Unit cell dimensions in model ethylene-butene-1 copolymers, Journal of Polymer Science Part B: Polymer Physics 27(11) (1989) 2269-2282.

[261] D.I. Bower, W.F. Maddams, The Vibrational Spectroscopy of Polymers, Cambridge University Press 1989.

[262] J.M. Lagaron, On the use of a Raman spectroscopy band to asses the crystalline lateral packing in polyethylene, Journal of Materials Science 37(19) (2002) 4101-4107.

[263] D. Galimberti, A. Milani, L. Maschio, C. Castiglioni, Intermolecular modulation of IR intensities in the solid state. The role of weak interactions in polyethylene crystal: A computational DFT study, The Journal of Chemical Physics 145(14) (2016) 144901.

[264] P.A.T. Olsson, E. Schröder, P. Hyldgaard, M. Kroon, E. Andreasson, E. Bergvall, Ab initio

and classical atomistic modelling of structure and defects in crystalline orthorhombic polyethylene: Twin boundaries, slip interfaces, and nature of barriers, Polymer 121(Supplement C) (2017) 234-246.

[265] E.B. Trigg, M.J. Stevens, K.I. Winey, Chain Folding Produces a Multilayered Morphology in a Precise Polymer: Simulations and Experiments, Journal of the American Chemical Society 139(10) (2017) 3747-3755.

[266] S. Hosoda, Y. Nozue, Y. Kawashima, K. Suita, S. Seno, T. Nagamatsu, K.B. Wagener, B. Inci, F. Zuluaga, G. Rojas, J.K. Leonard, Effect of the Sequence Length Distribution on the Lamellar Crystal Thickness and Thickness Distribution of Polyethylene: Perfectly Equisequential ADMET Polyethylene vs Ethylene/ $\alpha$-Olefin Copolymer, Macromolecules 44(2) (2011) 313-319.

[267] T.A. Kavassalis, P.R. Sundararajan, A molecular-dynamics study of polyethylene crystallization, Macromolecules 26(16) (1993) 4144-4150.

[268] P.R. Sundararajan, T.A. Kavassalis, Molecular dynamics study of polyethylene chain folding: the effects of chain length and the torsional barrier, Journal of the Chemical Society, Faraday Transactions 91(16) (1995) 2541-2549.

[269] S. Fujiwara, T. Sato, Molecular dynamics simulations of structural formation of a single polymer chain: Bond-orientational order and conformational defects, The Journal of Chemical Physics 107(2) (1997) 613-622.

[270] S. Fujiwara, T. Sato, Structure formation of a single polymer chain. I. Growth of trans domains, The Journal of Chemical Physics 114(14) (2001) 6455-6463.

[271] Q. Liao, X. Jin, Formation of segmental clusters during relaxation of a fully extended polyethylene chain at $300 \mathrm{~K}$ : A molecular dynamics simulation, The Journal of Chemical Physics 110(17) (1999) 8835-8841.

[272] T. Li, Z. Jiang, D. Yan, E. Nies, A polyethylene chain investigated with replica exchange molecular dynamics simulation: Equilibrium lamellar thickness and melting point, ordering and free energy, Polymer 51(23) (2010) 5612-5622.

[273] T. Li, X. Yang, E. Nies, A Replica Exchange Molecular Dynamics Simulation of a Single Polyethylene Chain: Temperature Dependence of Structural Properties and Chain

Conformational Study at the Equilibrium Melting Temperature, Journal of Chemical Theory and Computation 7(1) (2011) 188-202.

[274] W. Hu, D. Frenkel, V.B.F. Mathot, Free energy barrier to melting of single-chain polymer crystallite, The Journal of Chemical Physics 118(8) (2003) 3455-3457.

[275] W. Hu, D. Frenkel, V.B.F. Mathot, Intramolecular Nucleation Model for Polymer Crystallization, Macromolecules 36(21) (2003) 8178-8183.

[276] W.B. Hu, D. Frenkel, Effect of the Coil-Globule Transition on the Free-Energy Barrier for Intrachain Crystal Nucleation, The Journal of Physical Chemistry B 110(8) (2006) 3734-3737.

[277] H. Bu, Y. Pang, D. Song, T. Yu, T.M. Voll, G. Czornyj, B. Wunderlich, Single-molecule single crystals, Journal of Polymer Science Part B: Polymer Physics 29(2) (1991) 139-152.

[278] L. Liu, F. Su, H. Zhu, H. Li, E. Zhou, R. Yan, R. Qian, Single-chain single crystals of guttapercha, Journal of Macromolecular Science, Part B 36(2) (1997) 195-203. 
[279] M. Doran, P. Choi, Molecular dynamics studies of the effects of branching characteristics on the crystalline structure of polyethylene, The Journal of Chemical Physics 115(6) (2001) 2827-2830.

[280] X.-b. Zhang, Z.-s. Li, Z.-y. Lu, C.-c. Sun, Molecular dynamics simulation of the linear lowdensity polyethylene crystallization, The Journal of Chemical Physics 115(8) (2001) 3916-3922. [281] X.-b. Zhang, Z.-s. Li, H. Yang, C.-C. Sun, Molecular Dynamics Simulations on Crystallization of Polyethylene Copolymer with Precisely Controlled Branching, Macromolecules 37(19) (2004) 7393-7400.

[282] B.F. Sharkh, I.A. Hussein, MD Simulation Study of the Influence of Branch Content on Crystallization of Branched Polyethylene Chains with Uniform Branch Distribution, Journal of Polymer Research 11(3) (2004) 161-168.

[283] C. Liu, M. Muthukumar, Langevin dynamics simulations of early-stage polymer nucleation and crystallization, The Journal of Chemical Physics 109(6) (1998) 2536-2542.

[284] P. Welch, M. Muthukumar, Molecular Mechanisms of Polymer Crystallization from Solution, Physical Review Letters 87(21) (2001) 218302.

[285] L. Larini, A. Barbieri, D. Prevosto, P.A. Rolla, D. Leporini, Equilibrated polyethylene singlemolecule crystals: molecular-dynamics simulations and analytic model of the global minimum of the free-energy landscape, Journal of Physics: Condensed Matter 17(19) (2005) L199.

[286] L. Larini, D. Leporini, A manifestation of the Ostwald step rule: Molecular-dynamics simulations and free-energy landscape of the primary nucleation and melting of singlemolecule polyethylene in dilute solution, The Journal of Chemical Physics 123(14) (2005) 144907.

[287] L. Larini, D. Leporini, Free-energy effects in single-molecule polymer crystals, Journal of Non-Crystalline Solids 352(42) (2006) 5021-5024.

[288] J. Ramos, J. Martínez-Salazar, Computer modeling of the crystallization process of singlechain ethylene/1-hexene copolymers from dilute solutions, Journal of Polymer Science Part B: Polymer Physics 49(6) (2011) 421-430.

[289] S. Sanmartín, J. Ramos, J. Martínez-Salazar, Following the Crystallization Process of Polyethylene Single Chain by Molecular Dynamics: The Role of Lateral Chain Defects, Macromolecular Symposia 312(1) (2012) 97-107.

[290] R. Gao, X. He, Y. Shao, Y. Hu, H. Zhang, Z. Liu, B. Liu, Effects of Branch Content and Branch Length on Polyethylene Crystallization: Molecular Dynamics Simulation, Macromolecular Theory and Simulations 25(3) (2016) 303-311.

[291] R. Gao, X. He, H. Zhang, Y. Shao, Z. Liu, B. Liu, Molecular dynamics study of the isothermal crystallization mechanism of polyethylene chain: the combined effects of chain length and temperature, Journal of Molecular Modeling 22(3) (2016) 67.

[292] W. Hu, Chain folding in polymer melt crystallization studied by dynamic Monte Carlo simulations, The Journal of Chemical Physics 115(9) (2001) 4395-4401.

[293] G. Xu, W.L. Mattice, Study on structure formation of short polyethylene chains via dynamic Monte Carlo simulation, Computational and Theoretical Polymer Science 11(6) (2001) 405-413.

[294] W. Hu, Molecular Segregation in Polymer Melt Crystallization: Simulation Evidence and Unified-Scheme Interpretation, Macromolecules 38(21) (2005) 8712-8718.

[295] W. Hu, T. Cai, Regime Transitions of Polymer Crystal Growth Rates: Molecular Simulations and Interpretation beyond Lauritzen-Hoffman Model, Macromolecules 41(6) (2008) 2049-2061.

[296] W. Hu, V.B.F. Mathot, D. Frenkel, Phase Transitions of Bulk Statistical Copolymers Studied by Dynamic Monte Carlo Simulations, Macromolecules 36(6) (2003) 2165-2175. [297] H. Gao, M. Vadlamudi, R.G. Alamo, W. Hu, Monte Carlo Simulations of Strong Memory Effect of Crystallization in Random Copolymers, Macromolecules 46(16) (2013) 6498-6506. [298] N. Waheed, M.S. Lavine, G.C. Rutledge, Molecular simulation of crystal growth in neicosane, The Journal of Chemical Physics 116(5) (2002) 2301-2309. 
[299] N. Waheed, M.J. Ko, G.C. Rutledge, Molecular simulation of crystal growth in long alkanes, Polymer 46(20) (2005) 8689-8702.

[300] P. Yi, G.C. Rutledge, Molecular simulation of crystal nucleation in n-octane melts, The Journal of Chemical Physics 131(13) (2009) 134902.

[301] P. Yi, G.C. Rutledge, Molecular simulation of bundle-like crystal nucleation from neicosane melts, The Journal of Chemical Physics 135(2) (2011) 024903.

[302] N. Waheed, G.C. Rutledge, Crossover behavior in crystal growth rate from n-alkane to polyethylene, Journal of Polymer Science Part B: Polymer Physics 43(18) (2005) 2468-2473. [303] A. Bourque, C.R. Locker, G.C. Rutledge, Molecular Dynamics Simulation of Surface Nucleation during Growth of an Alkane Crystal, Macromolecules 49(9) (2016) 3619-3629. [304] A.J. Bourque, G.C. Rutledge, Kinetic Model for Layer-by-Layer Crystal Growth in Chain Molecules, Macromolecules 49(10) (2016) 3956-3964.

[305] M. Anwar, F. Turci, T. Schilling, Crystallization mechanism in melts of short $n$-alkane chains, The Journal of Chemical Physics 139(21) (2013) 214904.

[306] M. Anwar, J.T. Berryman, T. Schilling, Crystal nucleation mechanism in melts of short polymer chains under quiescent conditions and under shear flow, The Journal of Chemical Physics 141(12) (2014) 124910.

[307] M. Anwar, T. Schilling, Crystallization of polyethylene: A molecular dynamics simulation study of the nucleation and growth mechanisms, Polymer 76(Supplement C) (2015) 307-312. [308] R.H. Gee, N. Lacevic, L.E. Fried, Atomistic simulations of spinodal phase separation preceding polymer crystallization, Nat Mater 5(1) (2006) 39-43.

[309] N. Lacevic, L.E. Fried, R.H. Gee, Heterogeneous directional mobility in the early stages of polymer crystallization, The Journal of Chemical Physics 128(1) (2008) 014903.

[310] A. Koyama, T. Yamamoto, K. Fukao, Y. Miyamoto, Molecular dynamics studies on local ordering in amorphous polyethylene, The Journal of Chemical Physics 115(1) (2001) 560-566.

[311] A. Koyama, T. Yamamoto, K. Fukao, Y. Miyamoto, Molecular dynamics simulation of polymer crystallization from an oriented amorphous state, Physical Review E 65(5) (2002) 050801.

[312] A. Koyama, T. Yamamoto, K. Fukao, Y. Miyamoto, Molecular Dynamics Studies on Polymer Crystallization from a Stretched Amorphous State, Journal of Macromolecular Science, Part B 42(3-4) (2003) 821-831.

[313] T. Yamamoto, Molecular dynamics modeling of polymer crystallization from the melt, Polymer 45(4) (2004) 1357-1364.

[314] T. Yamamoto, Molecular dynamics simulations of steady-state crystal growth and homogeneous nucleation in polyethylene-like polymer, The Journal of Chemical Physics 129(18) (2008) 184903.

[315] T. Yamamoto, Molecular Dynamics of Reversible and Irreversible Melting in Chain-Folded Crystals of Short Polyethylene-like Polymer, Macromolecules 43(22) (2010) 9384-9393.

[316] T. Yamamoto, Molecular dynamics simulations of polymer crystallization in highly supercooled melt: Primary nucleation and cold crystallization, The Journal of Chemical Physics 133(3) (2010) 034904.

[317] T. Yamamoto, Molecular dynamics of polymer crystallization revisited: Crystallization from the melt and the glass in longer polyethylene, The Journal of Chemical Physics 139(5) (2013) 054903.

[318] A. Toda, K. Taguchi, K. Nozaki, M. Konishi, Melting behaviors of polyethylene crystals: An application of fast-scan DSC, Polymer 55(14) (2014) 3186-3194.

[319] M.J. Ko, N. Waheed, M.S. Lavine, G.C. Rutledge, Characterization of polyethylene crystallization from an oriented melt by molecular dynamics simulation, The Journal of Chemical Physics 121(6) (2004) 2823-2832.

[320] T. Yamamoto, Molecular dynamics in fiber formation of polyethylene and large deformation of the fiber, Polymer 54(12) (2013) 3086-3097. 
[321] G. UNGAR, J. STEJNY, A. KELLER, I. BIDD, M.C. WHITING, The Crystallization of Ultralong Normal Paraffins: The Onset of Chain Folding, Science 229(4711) (1985) 386-389.

[322] G. Ungar, A. Keller, Time-resolved synchrotron X-ray study of chain-folded crystallization of long paraffins, Polymer 27(12) (1986) 1835-1844.

[323] G. Ungar, X.B. Zeng, S.J. Spells, Non-integer and mixed integer forms in long $n$-alkanes observed by real-time LAM spectroscopy and SAXS, Polymer 41(25) (2000) 8775-8780.

[324] G. Ungar, S.J. Organ, A. Keller, X-ray evidence for sharp chain folds in crystalline linear alkanes, Journal of Polymer Science Part C: Polymer Letters 26(6) (1988) 259-262.

[325] B. Wunderlich, Macromolecular Physics, Elsevier Science2012.

[326] P.J. Barham, R.A. Chivers, A. Keller, J. Martinez-Salazar, S.J. Organ, The supercooling dependence of the initial fold length of polyethylene crystallized from the melt: unification of melt and solution crystallization, Journal of Materials Science 20(5) (1985) 1625-1630.

[327] J. Martinez-Salazar, P.J. Barham, A. Keller, The identification of the initial lamellar thickness of polyethylene crystals grown from the melt using synchrotron X-radiation, Journal of Materials Science 20(5) (1985) 1616-1624.

[328] P.J. Barham, R.A. Chivers, D.A. Jarvis, J. Martinez-Salazar, A. Keller, A new look at the crystallization of polyethylene. I. The initial fold length of melt-crystallized material, Journal of Polymer Science: Polymer Letters Edition 19(11) (1981) 539-547.

[329] R.A. Chivers, P.J. Barham, J. Martinez-Salazar, A. Keller, A new look at the crystallization of polyethylene. II. Crystallization from the melt at low supercoolings, Journal of Polymer Science: Polymer Physics Edition 20(9) (1982) 1717-1732.

[330] J. Martinez-Salazar, P.J. Barham, A. Keller, Studies on polyethylene crystallized at unusually high supercoolings: Fold length, habit, growth rate, epitaxy, Journal of Polymer Science: Polymer Physics Edition 22(6) (1984) 1085-1096.

[331] J.D. Hoffman, G.T. Davis, J.I. Lauritzen, The Rate of Crystallization of Linear Polymers with Chain Folding, in: N.B. Hannay (Ed.), Treatise on Solid State Chemistry: Volume 3 Crystalline and Noncrystalline Solids, Springer US, Boston, MA, 1976, pp. 497-614.

[332] C.H.M. Weber, A. Chiche, G. Krausch, S. Rosenfeldt, M. Ballauff, L. Harnau, I. GöttkerSchnetmann, Q. Tong, S. Mecking, Single Lamella Nanoparticles of Polyethylene, Nano Letters 7(7) (2007) 2024-2029.

[333] Y.-K. Lan, A.-C. Su, Nucleation of Polymer Crystals: The "ס Mystery", Macromolecules 43(19) (2010) 7908-7912.

[334] Y.-K. Lan, A.-C. Su, Primary nucleation of polyethylene: Embryogenesis from a semidilute solution, Polymer 55(14) (2014) 3087-3092.

[335] M. Sánchez Cuesta, J. Martinez Salazar, F.J. Baltá Calleja, X-Ray diffraction study of lattice distortions in branched polyethylene rapidly quenched from the melt, Polymer Bulletin 17(1) (1987) 23-30.

[336] J. Martinez-Salazar, J. Garc'ia Peña, F.J. Baltá Calleja, On the relation between hardness and intermolecular forces in polyethylene crystals, Polymer Communications 26 (1985) 57-59. [337] J. Martinez-Salazar, M. Sánchez Cuesta, F.J. Baltá Calleja, Equilibrium and nonequilibrium melting of branched polyethylene relating to defect incorporation within crystals, Colloid and Polymer Science 265(3) (1987) 239-245.

[338] M. Zhang, F. Yuen, P. Choi, Differences in the Solid-State Structures of Single-Site and Ziegler-Natta Linear Low-Density Polyethylenes As Revealed by Molecular Dynamics Simulation, Macromolecules 39(24) (2006) 8517-8525.

[339] C. Li, P. Choi, Molecular Dynamics Study of the Solid-State Structures of Linear-LowDensity Polyethylenes with Blocky Branches, Macromolecules 41(19) (2008) 7109-7114. [340] S. Sanmartín, J. Ramos, J.F. Vega, J. Martínez-Salazar, Strong influence of branching on the early stage of nucleation and crystal formation of fast cooled ultralong n-alkanes as revealed by computer simulation, European Polymer Journal 50(Supplement C) (2014) 190199. 
[341] G. Grasso, J.J. Titman, Chain Folding and Diffusion in Monodisperse Long n-Alkanes by Solid-State NMR, Macromolecules 42(12) (2009) 4175-4180.

[342] D.R. Rueda, J. Martinez-Salazar, F.J.B. Calleja, Lamellar diffusion during heat treatment in meltcrystallized low-density polyethylene, Journal of Polymer Science: Polymer Physics Edition 22(10) (1984) 1811-1820.

[343] D.R. Rueda, J. Martinez-Salazar, F.J. Baltá-Calleja, Annealing effects in lamellar linear polyethylene as revealed by microhardness, Journal of Materials Science 20(3) (1985) 834-838. [344] H. Meyer, F. Müller-Plathe, Formation of chain-folded structures in supercooled polymer melts, The Journal of Chemical Physics 115(17) (2001) 7807-7810.

[345] H. Meyer, F. Müller-Plathe, Formation of Chain-Folded Structures in Supercooled Polymer Melts Examined by MD Simulations, Macromolecules 35(4) (2002) 1241-1252.

[346] G.R. Strobl, The Physics of Polymers: Concepts for Understanding Their Structures and Behaviour, Springer1997.

[347] C. Luo, J.-U. Sommer, Coding coarse grained polymer model for LAMMPS and its application to polymer crystallization, Computer Physics Communications 180(8) (2009) 13821391.

[348] C. Luo, J.-U. Sommer, Coexistence of Melting and Growth during Heating of a Semicrystalline Polymer, Physical Review Letters 102(14) (2009) 147801.

[349] J.-U. Sommer, C. Luo, Molecular dynamics simulations of semicrystalline polymers: Crystallization, melting, and reorganization, Journal of Polymer Science Part B: Polymer Physics 48(21) (2010) 2222-2232.

[350] C. Luo, J.-U. Sommer, Growth Pathway and Precursor States in Single Lamellar Crystallization: MD Simulations, Macromolecules 44(6) (2011) 1523-1529.

[351] C. Luo, J.-U. Sommer, Disentanglement of Linear Polymer Chains Toward Unentangled Crystals, ACS Macro Letters 2(1) (2013) 31-34.

[352] C. Luo, J.-U. Sommer, Frozen Topology: Entanglements Control Nucleation and Crystallization in Polymers, Physical Review Letters 112(19) (2014) 195702.

[353] C. Luo, M. Kröger, J.-U. Sommer, Molecular dynamics simulations of polymer crystallization under confinement: Entanglement effect, Polymer 109(Supplement C) (2017) 71-84.

[354] C. Luo, J.-U. Sommer, Role of Thermal History and Entanglement Related Thickness Selection in Polymer Crystallization, ACS Macro Letters 5(1) (2016) 30-34.

[355] T. Vettorel, H. Meyer, Coarse Graining of Short Polythylene Chains for Studying Polymer Crystallization, Journal of Chemical Theory and Computation 2(3) (2006) 616-629.

[356] J. Ramos, J.F. Vega, S. Sanmartín, J. Martínez-Salazar, Coarse-grained simulations on the crystallization, melting and annealing processes of short chain branched polyolefins, European Polymer Journal 85(Supplement C) (2016) 478-488.

[357] T.Y. Cho, B. Heck, G. Strobl, Equations describing lamellar structure parameters and melting points of polyethylene-co-(butene/octene)s, Colloid and Polymer Science 282(8) (2004) 825-832.

[358] W.O. Statton, P.H. Geil, Recrystallization of polyethylene during annealing, Journal of Applied Polymer Science 3(9) (1960) 357-361.

[359] E.W. Fischer, G.F. Schmidt, Über Langperioden bei verstrecktem Polyäthylen, Angewandte Chemie 74(15) (1962) 551-562.

[360] A. Peterlin, Thickening of polymer single crystals during annealing, Journal of Polymer Science Part B: Polymer Letters 1(6) (1963) 279-284.

[361] F.R. Anderson, Morphology of Isothermally Bulk-Crystallized Linear Polyethylene, Journal of Applied Physics 35(1) (1964) 64-70.

[362] A. Peterlin, Crystallization and annealing of polyethylene, Die Makromolekulare Chemie 74(1) (1964) 107-128. 
[363] H.Y. Chen, S.P. Chum, A. Hiltner, E. Baer, Comparison of semicrystalline ethylene-styrene and ethylene-octene copolymers based on comonomer content, Journal of Polymer Science Part B: Polymer Physics 39(14) (2001) 1578-1593.

[364] A.M.E. Baker, A.H. Windle, Evidence for a partially ordered component in polyethylene from wide-angle X-ray diffraction, Polymer 42(2) (2001) 667-680.

[365] A. Alizadeh, A. Muñoz-Escalona, P. Lafuente, J.V. Garcia Ramos, J. Martínez-Salazar, The fine structure of metallocene-based linear polyethylenes: Part 1. A model grounded on molecular mobility, Polymer 40(15) (1999) 4345-4352.

[366] S. Martín, M.T. Expósito, J.F. Vega, J. Martínez-Salazar, Microstructure and properties of branched polyethylene: Application of a three-phase structural model, Journal of Applied Polymer Science 128(3) (2013) 1871-1878.

[367] M. Hütter, P.J. in 't Veld, G.C. Rutledge, Polyethylene $\{201\}$ crystal surface: interface stresses and thermodynamics, Polymer 47(15) (2006) 5494-5504.

[368] P.J. in 't Veld, M. Hütter, G.C. Rutledge, Temperature-Dependent Thermal and Elastic Properties of the Interlamellar Phase of Semicrystalline Polyethylene by Molecular Simulation, Macromolecules 39(1) (2006) 439-447.

[369] A. Ghazavizadeh, G.C. Rutledge, A.A. Atai, S. Ahzi, Y. Rémond, N. Soltani, Micromechanical characterization of the interphase layer in semi-crystalline polyethylene, Journal of Polymer Science Part B: Polymer Physics 51(16) (2013) 1228-1243.

[370] A. Galeski, Strength and toughness of crystalline polymer systems, Progress in Polymer Science 28(12) (2003) 1643-1699.

[371] S. Lee, G.C. Rutledge, Plastic Deformation of Semicrystalline Polyethylene by Molecular Simulation, Macromolecules 44(8) (2011) 3096-3108.

[372] I.-C. Yeh, J.W. Andzelm, G.C. Rutledge, Mechanical and Structural Characterization of Semicrystalline Polyethylene under Tensile Deformation by Molecular Dynamics Simulations, Macromolecules 48(12) (2015) 4228-4239.

[373] J.M. Kim, R. Locker, G.C. Rutledge, Plastic Deformation of Semicrystalline Polyethylene under Extension, Compression, and Shear Using Molecular Dynamics Simulation, Macromolecules 47(7) (2014) 2515-2528.

[374] I.-C. Yeh, J.L. Lenhart, G.C. Rutledge, J.W. Andzelm, Molecular Dynamics Simulation of the Effects of Layer Thickness and Chain Tilt on Tensile Deformation Mechanisms of Semicrystalline Polyethylene, Macromolecules 50(4) (2017) 1700-1712.

[375] F.J.B. Calleja, J.M. Salazar, H. Čačković, J. Loboda-Čačković, Correlation of hardness and microstructure in unoriented lamellar polyethylene, Journal of Materials Science 16(3) (1981) 739-751.

[376] J. Martinez Salazar, F.J. Baltá Calleja, Correlation of hardness and microstructure in unoriented lamellar polyethylene, Journal of Materials Science 18(4) (1983) 1077-1082. [377] J. Martinez-Salazar, F.J. Balta-Calleja, Mechanical model on polyethylene blends as revealed by microhardness, Journal of Materials Science Letters 4(3) (1985) 324-326.

[378] M.A. Kennedy, A.J. Peacock, L. Mandelkern, Tensile Properties of Crystalline Polymers: Linear Polyethylene, Macromolecules 27(19) (1994) 5297-5310.

[379] M.A. Kennedy, A.J. Peacock, M.D. Failla, J.C. Lucas, L. Mandelkern, Tensile Properties of Crystalline Polymers: Random Copolymers of Ethylene, Macromolecules 28(5) (1995) 14071421.

[380] F. Nilsson, X. Lan, T. Gkourmpis, M.S. Hedenqvist, U.W. Gedde, Modelling tie chains and trapped entanglements in polyethylene, Polymer 53(16) (2012) 3594-3601.

[381] A. Moyassari, H. Mostafavi, T. Gkourmpis, M.S. Hedenqvist, U.W. Gedde, F. Nilsson, Simulation of semi-crystalline polyethylene: Effect of short-chain branching on tie chains and trapped entanglements, Polymer 72(Supplement C) (2015) 177-184.

[382] V. Kumar, C.R. Locker, P.J. in 't Veld, G.C. Rutledge, Effect of Short Chain Branching on the Interlamellar Structure of Semicrystalline Polyethylene, Macromolecules 50(3) (2017) 1206-1214. 
[383] Y.-L. Huang, N. Brown, Dependence of slow crack growth in polyethylene on butyl branch density: Morphology and theory, Journal of Polymer Science Part B: Polymer Physics 29(1) (1991) 129-137.

[384] S.C. Glotzer, W. Paul, Molecular and Mesoscale Simulation Methods for Polymer Materials, Annual Review of Materials Research 32(1) (2002) 401-436.

[385] K. Kremer, Computer Simulations for Macromolecular Science, Macromolecular Chemistry and Physics 204(2) (2003) 257-264.

[386] F. Müller-Plathe, Scale-Hopping in Computer Simulations of Polymers, Soft Materials 1(1) (2002) 1-31.

[387] C. Lorenz, N.L. Doltsinis, Molecular Dynamics Simulation: From "Ab Initio" to "Coarse Grained", in: J. Leszczynski, A. Kaczmarek-Kedziera, T. Puzyn, M. G. Papadopoulos, H. Reis, M. K. Shukla (Eds.), Handbook of Computational Chemistry, Springer International Publishing, Cham, 2017, pp. 337-396.

[388] A. Szabo, N.S. Ostlund, Modern Quantum Chemistry: Introduction to Advanced Electronic Structure Theory, Dover Publications1989.

[389] J.M. Seminario, An introduction to density functional theory in chemistry, in: J.M.

Seminario, P. Politzer (Eds.), Theoretical and Computational Chemistry, Elsevier1995, pp. 1-27. [390] D.S. Sholl, J.A. Steckel, Density Functional Theory: A Practical Introduction, John Wiley \& Sons2009.

[391] A.C. Tsipis, DFT flavor of coordination chemistry, Coordination Chemistry Reviews 272 (2014) 1-29.

[392] D.C. Young, COMPUTATIONAL CHEMISTRY: A Practical Guide for Applying Techniques to Real-World Problems, John Wiley \& Sons, Inc.2002, pp. 78-92, 231-238, 268-269.

[393] S.F. Sousa, P.A. Fernandes, M.J. Ramos, General Performance of Density Functionals, The Journal of Physical Chemistry A 111(42) (2007) 10439-10452.

[394] Wikipedia, https://en.wikipedia.org/wiki/List of quantum chemistry and solidstate physics software. 2017).

[395] S. Pirhadi, J. Sunseri, D.R. Koes, Open source molecular modeling, Journal of Molecular Graphics and Modelling 69 (2016) 127-143.

[396] J.C.L. Hageman, R.J. Meier, M. Heinemann, R.A. de Groot, Young Modulus of Crystalline Polyethylene from ab Initio Molecular Dynamics, Macromolecules 30(19) (1997) 5953-5957.

[397] J.C.L. Hageman, G.A. de Wijs, R.A. de Groot, R.J. Meier, Bond Scission in a Perfect

Polyethylene Chain and the Consequences for the Ultimate Strength, Macromolecules 33(24)

(2000) 9098-9108.

[398] F. Bartha, F. Bogár, A. Peeters, C. Van Alsenoy, V. Van Doren, Density-functional calculations of the elastic properties of some polymer chains, Physical Review B 62(15) (2000) 10142-10150.

[399] S. Suhai, Ab initio calculation of polyethylene deformation including electron correlation effects, Journal of Polymer Science: Polymer Physics Edition 21(8) (1983) 1341-1346.

[400] B. Crist, P.G. Hereña, Molecular orbital studies of polyethylene deformation, Journal of Polymer Science Part B: Polymer Physics 34(3) (1996) 449-457.

[401] A. Karpfen, Ab initio studies on polymers. V. All-trans-polyethylene, The Journal of Chemical Physics 75(1) (1981) 238-245.

[402] J.C.L. Hageman, R.A. de Groot, R.J. Meier, Ultra strong polymer fibers: Ab initio calculations on polyethylene, Computational Materials Science 10(1) (1998) 180-183.

[403] S. Suhai, Quantum mechanical calculation of the longitudinal elastic modulus and of deviations from Hooke's law in polyethylene, The Journal of Chemical Physics 84(9) (1986) 5071-5076.

[404] D.S. Boudreaux, Calculations of the strength of the polyethylene molecule, Journal of Polymer Science: Polymer Physics Edition 11(7) (1973) 1285-1292.

[405] J.C.L. Hageman, G.A. de Wijs, R.A. de Groot, R.J. Meier, Weakening of a Polyethylene Chain by Methyl Side Groups, Soft Materials 1(2) (2003) 223-233. 
[406] M.L. Zhang, M.S. Miao, A. Peeters, C. Van Alsenoy, J.J. Ladik, V.E. Van Doren, LDA calculations of the Young's moduli of polyethylene and six polyfluoroethylenes, Solid State Communications 116(6) (2000) 339-343.

[407] M.S. Miao, P.E. Van Camp, V.E. Van Doren, J.J. Ladik, J.W. Mintmire, Conformation and electronic structure of polyethylene: A density-functional approach, Physical Review B 54(15) (1996) 10430-10435.

[408] M.S. Miao, M.L. Zhang, V.E. Van Doren, C. Van Alsenoy, J.L. Martins, Density functional calculations on the structure of crystalline polyethylene under high pressures, The Journal of Chemical Physics 115(24) (2001) 11317-11324.

[409] B. Montanari, R.O. Jones, Density functional study of crystalline polyethylene, Chemical Physics Letters 272(5) (1997) 347-352.

[410] M. Meunier, N. Quirke, Molecular modeling of electron trapping in polymer insulators, The Journal of Chemical Physics 113(1) (2000) 369-376.

[411] H. Teramae, T. Yamabe, A. Imamura, Ab initio effective core potential studies on polymers, Theoretica chimica acta 64(1) (1983) 1-12.

[412] M. Springborg, M. Lev, Electronic structures of polyethylene and

polytetrafluoroethylene, Physical Review B 40(5) (1989) 3333-3339.

[413] S. Serra, E. Tosatti, S. Iarlori, S. Scandolo, G. Santoro, Interchain electron states in polyethylene, Physical Review B 62(7) (2000) 4389-4393.

[414] P. Fabien, S. Alexander, C. Andrea Dal, T. Erio, Complex band structures and decay length in polyethylene chains, Journal of Physics: Condensed Matter 15(22) (2003) 3731.

[415] C.C. Wang, G. Pilania, R. Ramprasad, Dielectric properties of carbon-, silicon-, and germanium-based polymers: A first-principles study, Physical Review B 87(3) (2013) 035103.

[416] G. Pilania, C.C. Wang, K. Wu, N. Sukumar, C. Breneman, G. Sotzing, R. Ramprasad, New Group IV Chemical Motifs for Improved Dielectric Permittivity of Polyethylene, Journal of Chemical Information and Modeling 53(4) (2013) 879-886.

[417] Z. Jing, C. Li, H. Zhao, G. Zhang, B. Han, Doping Effect of Graphene Nanoplatelets on Electrical Insulation Properties of Polyethylene: From Macroscopic to Molecular Scale, Materials 9(8) (2016) 680.

[418] E. Talebian, M. Talebian, A comparative DFT study on the differences between normal modes of polyethylene and polyethylene glycol via B3LYP Hamiltonian and the Hartree-Fock method in multiple bases, Optik - International Journal for Light and Electron Optics 125(1) (2014) 228-231.

[419] D.A. Braden, S.F. Parker, J. Tomkinson, B.S. Hudson, Inelastic neutron scattering spectra of the longitudinal acoustic modes of the normal alkanes from pentane to pentacosane, The Journal of Chemical Physics 111(1) (1999) 429-437.

[420] T.R. Mattsson, J.M.D. Lane, K.R. Cochrane, M.P. Desjarlais, A.P. Thompson, F. Pierce, G.S. Grest, First-principles and classical molecular dynamics simulation of shocked polymers, Physical Review B 81(5) (2010) 054103.

[421] T.L. Chantawansri, T.W. Sirk, E.F.C. Byrd, J.W. Andzelm, B.M. Rice, Shock Hugoniot calculations of polymers using quantum mechanics and molecular dynamics, The Journal of Chemical Physics 137(20) (2012) 204901.

[422] T. Schlick, Molecular Modeling and Simulation: An Interdisciplinary Guide, 2nd Edition, Springer Science2010.

[423] P. Choi, Q. Wang, E. Vignola, Molecular dynamics study of the conformation and dynamics of precisely branched polyethylene, Polymer 55(22) (2014) 5734-5738.

[424] P.V.K. Pant, J. Han, G.D. Smith, R.H. Boyd, A molecular dynamics simulation of polyethylene, The Journal of Chemical Physics 99(1) (1993) 597-604.

[425] L. Verlet, Computer "Experiments" on Classical Fluids. I. Thermodynamical Properties of Lennard-Jones Molecules, Physical Review 159(1) (1967) 98-103. 
[426] B.G. Sumpter, D.W. Noid, B. Wunderlich, S.Z.D. Cheng, Molecular dynamics study of the rate of melting of a crystalline polyethylene molecule: effect of chain folding, Macromolecules 23(21) (1990) 4671-4677.

[427] M.S. Green, Markoff Random Processes and the Statistical Mechanics of TimeDependent Phenomena. II. Irreversible Processes in Fluids, The Journal of Chemical Physics 22(3) (1954) 398-413.

[428] R. Kubo, M. Yokota, S. Nakajima, Statistical-Mechanical Theory of Irreversible Processes. II. Response to Thermal Disturbance, Journal of the Physical Society of Japan 12(11) (1957) 1203-1211.

[429] D.J. Evans, G.P. Morriss, Nonlinear-response theory for steady planar Couette flow, Physical Review A 30(3) (1984) 1528-1530.

[430] Dassault Systèmes BIOVIA http://accelrys.com/products/collaborative-science/bioviamaterials-studio/. 2017).

[431] M.J. Abraham, T. Murtola, R. Schulz, S. Páll, J.C. Smith, B. Hess, E. Lindahl, GROMACS: High performance molecular simulations through multi-level parallelism from laptops to supercomputers, SoftwareX 1-2 (2015) 19-25.

[432] S. Plimpton, Fast Parallel Algorithms for Short-Range Molecular Dynamics, Journal of Computational Physics 117(1) (1995) 1-19.

[433] J. Glaser, T.D. Nguyen, J.A. Anderson, P. Lui, F. Spiga, J.A. Millan, D.C. Morse, S.C. Glotzer, Strong scaling of general-purpose molecular dynamics simulations on GPUs, Computer Physics Communications 192 (2015) 97-107.

[434] N. Metropolis, A.W. Rosenbluth, M.N. Rosenbluth, A.H. Teller, E. Teller, Equation of State Calculations by Fast Computing Machines, The Journal of Chemical Physics 21(6) (1953) 10871092.

[435] M.N. Rosenbluth, A.W. Rosenbluth, Monte Carlo Calculation of the Average Extension of Molecular Chains, The Journal of Chemical Physics 23(2) (1955) 356-359.

[436] D. Frenkel, B. Smit, Chapter 13 - Biased Monte Carlo Schemes, Understanding Molecular Simulation (Second Edition), Academic Press, San Diego, 2002, pp. 321-387.

[437] D. Frenkel, B. Smit, Chapter 5 - Monte Carlo Simulations in Various Ensembles, Understanding Molecular Simulation (Second Edition), Academic Press, San Diego, 2002, pp. 111-137.

[438] D. Dubbeldam, A. Torres-Knoop, K.S. Walton, On the inner workings of Monte Carlo codes, Molecular Simulation 39(14-15) (2013) 1253-1292.

[439] D.N. Theodorou, Progress and Outlook in Monte Carlo Simulations, Industrial \& Engineering Chemistry Research 49(7) (2010) 3047-3058.

[440] J.K. Shah, E.J. Maginn, A general and efficient Monte Carlo method for sampling intramolecular degrees of freedom of branched and cyclic molecules, The Journal of Chemical Physics 135(13) (2011) 134121.

[441] J.K. Shah, E. Marin-Rimoldi, R.G. Mullen, B.P. Keene, S. Khan, A.S. Paluch, N. Rai, L.L. Romanielo, T.W. Rosch, B. Yoo, E.J. Maginn, Cassandra: An open source Monte Carlo package for molecular simulation, Journal of Computational Chemistry 38(19) (2017) 1727-1739. [442] M.G. Martin, MCCCS Towhee: a tool for Monte Carlo molecular simulation, Molecular Simulation 39(14-15) (2013) 1212-1222.

[443] P. Doruker, W.L. Mattice, Dynamics of bulk polyethylene on a high coordination lattice, Macromolecular Symposia 133(1) (1998) 47-70.

[444] R.F. Rapold, W.L. Mattice, New high-coordination lattice model for rotational isomeric state polymer chains, Journal of the Chemical Society, Faraday Transactions 91(16) (1995) 2435-2441.

[445] G. Xu, W.L. Mattice, Monte Carlo simulation of the crystallization and annealing of a freestanding thin film of n-tetracontane, The Journal of Chemical Physics 116(5) (2002) 22772283. 
[446] H. Lin, W.L. Mattice, E.D. Von Meerwall, Dynamics of polyethylene melts studied by Monte Carlo simulations on a high coordination lattice, Journal of Polymer Science Part B: Polymer Physics 44(18) (2006) 2556-2571.

[447] I. Carmesin, K. Kremer, The bond fluctuation method: a new effective algorithm for the dynamics of polymers in all spatial dimensions, Macromolecules 21(9) (1988) 2819-2823.

[448] V. Tries, W. Paul, J. Baschnagel, K. Binder, Modeling polyethylene with the bond fluctuation model, The Journal of Chemical Physics 106(2) (1997) 738-748.

[449] R. Auhl, R. Everaers, G.S. Grest, K. Kremer, S.J. Plimpton, Equilibration of long chain polymer melts in computer simulations, The Journal of Chemical Physics 119(24) (2003) 1271812728.

[450] Y.R. Sliozberg, M. Kröger, T.L. Chantawansri, Fast equilibration protocol for million atom systems of highly entangled linear polyethylene chains, The Journal of Chemical Physics 144(15) (2016) 154901.

[451] G. Zhang, L.A. Moreira, T. Stuehn, K.C. Daoulas, K. Kremer, Equilibration of High Molecular Weight Polymer Melts: A Hierarchical Strategy, ACS Macro Letters 3(2) (2014) 198203.

[452] T. Vettorel, G. Besold, K. Kremer, Fluctuating soft-sphere approach to coarse-graining of polymer models, Soft Matter 6(10) (2010) 2282-2292.

[453] C. Svaneborg, H.A. Karimi-Varzaneh, N. Hojdis, F. Fleck, R. Everaers, Multiscale approach to equilibrating model polymer melts, Physical Review E 94(3) (2016) 032502.

[454] G. Milano, T. Kawakatsu, Hybrid particle-field molecular dynamics simulations for dense polymer systems, The Journal of Chemical Physics 130(21) (2009) 214106.

[455] Y. Zhao, A. De Nicola, T. Kawakatsu, G. Milano, Hybrid particle-field molecular dynamics simulations: Parallelization and benchmarks, Journal of Computational Chemistry 33(8) (2012) 868-880.

[456] A. De Nicola, T. Kawakatsu, G. Milano, Generation of Well-Relaxed All-Atom Models of Large Molecular Weight Polymer Melts: A Hybrid Particle-Continuum Approach Based on Particle-Field Molecular Dynamics Simulations, Journal of Chemical Theory and Computation 10(12) (2014) 5651-5667.

[457] W.L. Jorgensen, D.S. Maxwell, J. Tirado-Rives, Development and Testing of the OPLS AllAtom Force Field on Conformational Energetics and Properties of Organic Liquids, Journal of the American Chemical Society 118(45) (1996) 11225-11236.

[458] M.L.P. Price, D. Ostrovsky, W.L. Jorgensen, Gas-phase and liquid-state properties of esters, nitriles, and nitro compounds with the OPLS-AA force field, Journal of Computational Chemistry 22(13) (2001) 1340-1352.

[459] S.W.I. Siu, K. Pluhackova, R.A. Böckmann, Optimization of the OPLS-AA Force Field for Long Hydrocarbons, Journal of Chemical Theory and Computation 8(4) (2012) 1459-1470.

[460] L.R. Dodd, D.N. Theodorou, Atomistic Monte Carlo simulation and continuum mean field theory of the structure and equation of state properties of alkane and polymer melts, in: L. Monnerie, U.W. Suter (Eds.), Atomistic Modeling of Physical Properties, Springer Berlin Heidelberg, Berlin, Heidelberg, 1994, pp. 249-281.

[461] P. van der Ploeg, H.J.C. Berendsen, Molecular dynamics simulation of a bilayer membrane, The Journal of Chemical Physics 76(6) (1982) 3271-3276.

[462] J.P. Ryckaert, A. Bellemans, Molecular dynamics of liquid n-butane near its boiling point, Chemical Physics Letters 30(1) (1975) 123-125.

[463] S. Toxvaerd, Equation of state of alkanes II, The Journal of Chemical Physics 107(13) (1997) 5197-5204.

[464] S.L. Mayo, B.D. Olafson, W.A. Goddard, DREIDING: a generic force field for molecular simulations, The Journal of Physical Chemistry 94(26) (1990) 8897-8909.

[465] M.S. Lavine, N. Waheed, G.C. Rutledge, Molecular dynamics simulation of orientation and crystallization of polyethylene during uniaxial extension, Polymer 44(5) (2003) 1771-1779. 
[466] H. Sun, COMPASS: An ab Initio Force-Field Optimized for Condensed-Phase ApplicationsOverview with Details on Alkane and Benzene Compounds, The Journal of Physical Chemistry B 102(38) (1998) 7338-7364.

[467] C. Li, P. Choi, P.R. Sundararajan, Simulation of chain folding in polyethylene: A comparison of united atom and explicit hydrogen atom models, Polymer 51(13) (2010) 28032808.

[468] A.L. Brayton, I.-C. Yeh, J.W. Andzelm, G.C. Rutledge, Vibrational Analysis of Semicrystalline Polyethylene Using Molecular Dynamics Simulation, Macromolecules 50(17) (2017) 6690-6701.

[469] P. Carbone, H.A.K. Varzaneh, X. Chen, F. Müller-Plathe, Transferability of coarse-grained force fields: The polymer case, The Journal of Chemical Physics 128(6) (2008) 064904.

[470] T.C. Moore, C.R. lacovella, C. McCabe, Derivation of coarse-grained potentials via multistate iterative Boltzmann inversion, The Journal of Chemical Physics 140(22) (2014) 224104.

[471] M.G. Guenza, Theoretical models for bridging timescales in polymer dynamics, Journal of Physics: Condensed Matter 20(3) (2008) 033101.

[472] V. Rühle, C. Junghans, A. Lukyanov, K. Kremer, D. Andrienko, Versatile Object-Oriented Toolkit for Coarse-Graining Applications, Journal of Chemical Theory and Computation 5(12) (2009) 3211-3223.

[473] L.-J. Chen, H.-J. Qian, Z.-Y. Lu, Z.-S. Li, C.-C. Sun, An Automatic Coarse-Graining and FineGraining Simulation Method: Application on Polyethylene, The Journal of Physical Chemistry B 110(47) (2006) 24093-24100.

[474] T.W. Sirk, Y.R. Slizoberg, J.K. Brennan, M. Lisal, J.W. Andzelm, An enhanced entangled polymer model for dissipative particle dynamics, The Journal of Chemical Physics 136(13) (2012) 134903.

[475] H. Fukunaga, J.-i. Takimoto, M. Doi, A coarse-graining procedure for flexible polymer chains with bonded and nonbonded interactions, The Journal of Chemical Physics 116(18) (2002) 8183-8190.

[476] J.T. Padding, W.J. Briels, Zero-shear stress relaxation and long time dynamics of a linear polyethylene melt: A test of Rouse theory, The Journal of Chemical Physics 114(19) (2001) 8685-8693.

[477] L. Liu, J.T. Padding, W.K. den Otter, W.J. Briels, Coarse-grained simulations of moderately entangled star polyethylene melts, The Journal of Chemical Physics 138(24) (2013) 244912. [478] J. McCarty, I.Y. Lyubimov, M.G. Guenza, Multiscale Modeling of Coarse-Grained Macromolecular Liquids, The Journal of Physical Chemistry B 113(35) (2009) 11876-11886. [479] K.S. Schweizer, K.G. Honnell, J.G. Curro, Reference interaction site model theory of polymeric liquids: Self-consistent formulation and nonideality effects in dense solutions and melts, The Journal of Chemical Physics 96(4) (1992) 3211-3225.

[480] J. McCarty, A.J. Clark, J. Copperman, M.G. Guenza, An analytical coarse-graining method which preserves the free energy, structural correlations, and thermodynamic state of polymer melts from the atomistic to the mesoscale, The Journal of Chemical Physics 140(20) (2014) 204913.

[481] Q. Wang, D. Yang, The recent structure-based coarse graining of polymer melts using PRISM theory does not give thermodynamic consistency, Polymer 111(Supplement C) (2017) 103-106.

[482] M. Dinpajooh, M.G. Guenza, Thermodynamic consistency in the structure-based integral equation coarse-grained method, Polymer 117 (2017) 282-286.

[483] I.Y. Lyubimov, J. McCarty, A. Clark, M.G. Guenza, Analytical rescaling of polymer dynamics from mesoscale simulations, The Journal of Chemical Physics 132(22) (2010) 224903. [484] P. Kindt, W.J. Briels, A single particle model to simulate the dynamics of entangled polymer melts, The Journal of Chemical Physics 127(13) (2007) 134901.

[485] W.J. Briels, Transient forces in flowing soft matter, Soft Matter 5(22) (2009) 4401-4411. 
[486] P.J. Hoogerbrugge, J.M.V.A. Koelman, Simulating Microscopic Hydrodynamic Phenomena with Dissipative Particle Dynamics, EPL (Europhysics Letters) 19(3) (1992) 155.

[487] P. Español, P. Warren, Statistical Mechanics of Dissipative Particle Dynamics, EPL (Europhysics Letters) 30(4) (1995) 191.

[488] R.D. Groot, P.B. Warren, Dissipative particle dynamics: Bridging the gap between atomistic and mesoscopic simulation, The Journal of Chemical Physics 107(11) (1997) 4423-

4435.

[489] W.-J. Lee, S.-P. Ju, Y.-C. Wang, J.-G. Chang, Modeling of polyethylene and poly (L-lactide) polymer blends and diblock copolymer: Chain length and volume fraction effects on structural arrangement, The Journal of Chemical Physics 127(6) (2007) 064902.

[490] J.-G. Gai, H.-L. Li, C. Schrauwen, G.-H. Hu, Dissipative particle dynamics study on the phase morphologies of the ultrahigh molecular weight

polyethylene/polypropylene/poly(ethylene glycol) blends, Polymer 50(1) (2009) 336-346. [491] J.-G. Gai, G.-H. Hu, H.-L. Li, S.-P. Zhu, S. Hoppe, Dissipative Particle Dynamics and Flory-Huggins Theories for Predicting the Rheological Behavior of Ultrahigh Molecular Weight Polyethylene Blends, Industrial \& Engineering Chemistry Research 49(22) (2010) 11369-11379. [492] Y.-C. Wang, S.-P. Ju, H.-Z. Cheng, J.-M. Lu, H.-H. Wang, Modeling of Polyethylene and Functionalized CNT Composites: A Dissipative Particle Dynamics Study, The Journal of Physical Chemistry C 114(8) (2010) 3376-3384.

[493] Y.-C. Wang, S.-P. Ju, T.J. Huang, H.-H. Wang, Modeling of polyethylene, poly(l-lactide), and CNT composites: a dissipative particle dynamics study, Nanoscale Research Letters 6(1) (2011) 433.

[494] X. Guerrault, B. Rousseau, J. Farago, Dissipative particle dynamics simulations of polymer melts. I. Building potential of mean force for polyethylene and cis-polybutadiene, The Journal of Chemical Physics 121(13) (2004) 6538-6546.

[495] F. Lahmar, B. Rousseau, Influence of the adjustable parameters of the DPD on the global and local dynamics of a polymer melt, Polymer 48(12) (2007) 3584-3592.

[496] F. Lahmar, C. Tzoumanekas, D.N. Theodorou, B. Rousseau, Onset of Entanglements Revisited. Dynamical Analysis, Macromolecules 42(19) (2009) 7485-7494.

[497] C. Tzoumanekas, F. Lahmar, B. Rousseau, D.N. Theodorou, Onset of Entanglements Revisited. Topological Analysis, Macromolecules 42(19) (2009) 7474-7484.

[498] S. Kumar, R.G. Larson, Brownian dynamics simulations of flexible polymers with springspring repulsions, The Journal of Chemical Physics 114(15) (2001) 6937-6941.

[499] G. PAN, C.W. MANKE, DEVELOPMENTS TOWARD SIMULATION OF ENTANGLED POLYMER MELTS BY DISSIPATIVE PARTICLE DYNAMICS (DPD), International Journal of Modern Physics B 17(01n02) (2003) 231-235.

[500] M. Yamanoi, O. Pozo, J.M. Maia, Linear and non-linear dynamics of entangled linear polymer melts by modified tunable coarse-grained level Dissipative Particle Dynamics, The Journal of Chemical Physics 135(4) (2011) 044904.

[501] P. Nikunen, I. Vattulainen, M. Karttunen, Reptational dynamics in dissipative particle dynamics simulations of polymer melts, Physical Review E 75(3) (2007) 036713.

[502] A.A. Gavrilov, A.V. Chertovich, P.G. Khalatur, A.R. Khokhlov, Study of the Mechanisms of Filler Reinforcement in Elastomer Nanocomposites, Macromolecules 47(15) (2014) 5400-5408. [503] M. Mohagheghi, B. Khomami, Elucidating the flow-microstructure coupling in the entangled polymer melts. Part I: Single chain dynamics in shear flow, Journal of Rheology 60(5) (2016) 849-859.

[504] A. Gooneie, S. Schuschnigg, C. Holzer, Coupled Orientation and Stretching of Chains in Mesoscale Models of Polydisperse Linear Polymers in Startup of Steady Shear Flow Simulations, Macromolecular Theory and Simulations 25(2) (2016) 170-186.

[505] Y.-L. Zhu, H. Liu, Z.-Y. Lu, A highly coarse-grained model to simulate entangled polymer melts, The Journal of Chemical Physics 136(14) (2012) 144903. 
[506] M. Praprotnik, L.D. Site, K. Kremer, Multiscale Simulation of Soft Matter: From Scale Bridging to Adaptive Resolution, Annual Review of Physical Chemistry 59(1) (2008) 545-571. [507] S.O. Nielsen, R.E. Bulo, P.B. Moore, B. Ensing, Recent progress in adaptive multiscale molecular dynamics simulations of soft matter, Physical Chemistry Chemical Physics 12(39) (2010) 12401-12414.

[508] M. Praprotnik, L. Delle Site, K. Kremer, Adaptive resolution molecular-dynamics simulation: Changing the degrees of freedom on the fly, The Journal of Chemical Physics 123(22) (2005) 224106.

[509] S.O. Nielsen, P.B. Moore, B. Ensing, Adaptive Multiscale Molecular Dynamics of Macromolecular Fluids, Physical Review Letters 105(23) (2010) 237802.

[510] M. Praprotnik, S. Poblete, L. Delle Site, K. Kremer, Comment on "Adaptive Multiscale Molecular Dynamics of Macromolecular Fluids", Physical Review Letters 107(9) (2011) 099801. [511] N. di Pasquale, D. Marchisio, P. Carbone, Mixing atoms and coarse-grained beads in modelling polymer melts, The Journal of Chemical Physics 137(16) (2012) 164111. 

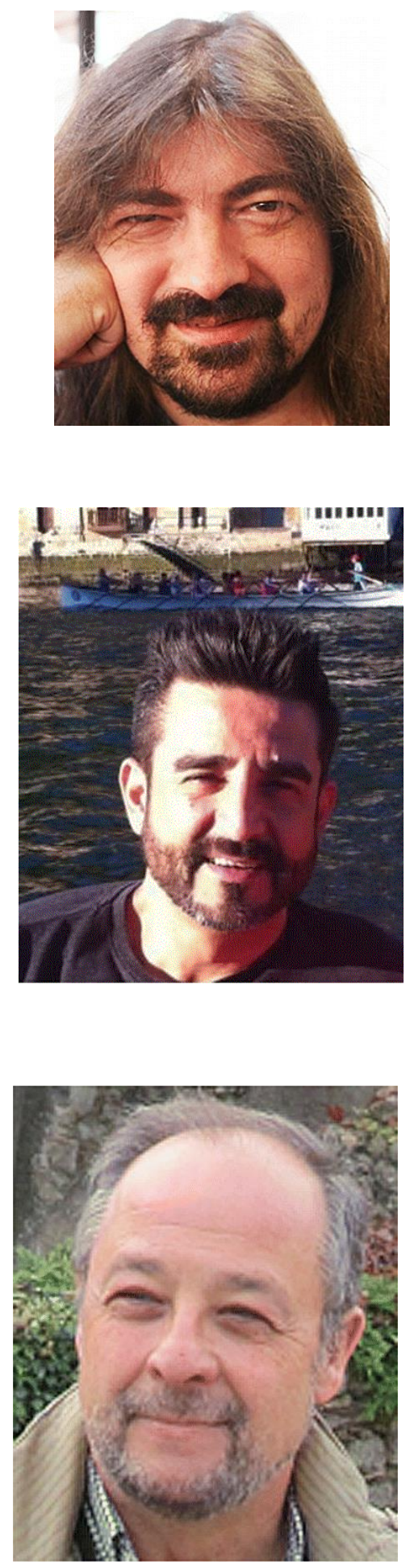

Javier Ramos holds a degree in Chemistry (Alcala University, 1998) and a degree in Technical Engineering in Computer Systems (UNED, 2011). He received his Ph.D. from Alcala University in 2002, working on computer simulations to examine olefin polymerization mechanisms using single-site catalysts in collaboration with the oil company Repsol-YPF. From 2005, he spent 2 years at Professor Theodorou's laboratory, Athens University investigating the molten properties of polyolefins as a function of their molecular architecture using Monte Carlo and molecular dynamics simulations. He was awarded a Ramón y Cajal Fellowship in 2012. Presently, Dr. Ramos is a researcher at the Institute of the Structure of Matter (IEM-CSIC, Madrid). His current research interests are modelling and simulation studies in both synthetic and biological macromolecular systems.

Juan F. Vega obtained his degree in Macromolecular Chemistry in 1992 and his PhD in Chemistry in 1998 at the University of Basque Country (UPV/EHU, San Sebastián). He then spent three years at the Institute of the Structure of Matter (IEM-CSIC, Madrid) working as a Postdoc on rheology and solid state properties of polymers. In 2002, he left Madrid to take on a Postdoc position at Teschniche Universiteit Eindhoven (TU/e, The Netherlands) where he continued working on the rheological properties of polymers. In 2006, he received a Ramón y Cajal Fellowship, and in 2008 he returned to the IEM-CSIC where he now holds a position as research scientist. Dr. Vega is currently working on melt polymer dynamics, polymer crystallization and hydrodynamics of (bio)macromolecules combining experiments and simulations.

Prof. Javier Martinez-Salazar obtained his $\mathrm{PhD}$ at the Universidad Autónoma de Madrid in 1979 on the X-ray diffraction and viscoelasticity of polymers. He then took on a postdoctoral position with the group of Prof. Keller at H.H. Wills Physics laboratory in Bristol, UK, where he specialized in various aspects of polymer physics. He is a member of the EPF and has served as representative of the Spanish polymer group over the period 1998-2010. He has earned numerous contracts with industry in the area of polyolefins, in particular with the Spanish oil company RepsolYPF, helping to build the well-known polymer chain of knowledge - from design to product. Presently he is a Research Professor at the Institute of the Structure of Matter (IEM-CSIC, Madrid) where he is heading the group Biophym. 
